\title{
IRREDUCIBLE TENSOR PRODUCTS OF REPRESENTATIONS OF COVERING GROUPS OF SYMMETRIC AND ALTERNATING GROUPS
}

\author{
LUCIA MOROTTI
}

ABSTRACT. In this paper we completely classify irreducible tensor products of covering groups of symmetric and alternating groups in characteristic $\neq 2$.

\section{INTRODUCTION}

Let $F$ be an algebraically closed filed, $G$ be a group and $V$ and $W$ be irreducible $F G$-representation. A natural question to ask is when the tensor product $V \otimes W$ is irreducible. This is always the case if $V$ or $W$ is 1-dimensional, so the interesting cases are those where neither $V$ nor $W$ is 1-dimensional but $V \otimes W$ is irreducible, in which case we say that $V \otimes W$ is a non-trivial irreducible tensor product. One motivation to this question comes from the Aschbacher-Scott classification of maximal subgroups of finite classical groups; see [1,2].

Irreducible tensor products of symmetric groups have been fully classified in 6, 19, 20, 36, 46. For alternating groups, apart for some cases in in characteristic 2, non-trivial tensor products have been classified in [5, 7, 37, 38, 46]. For covering groups of symmetric and alternating groups however only partial results are known, that is the characteristic 0 case for $\widetilde{\mathrm{S}}_{n}$, see [4,8], as well as some reduction results obtained in 34 for $\widetilde{\mathrm{S}}_{n}$ and $\widetilde{\mathrm{A}}_{n}$ in characteristic $\geq 5$. In this paper we will consider the case where $G=\widetilde{\mathrm{S}}_{n}$ or $\widetilde{\mathrm{A}}_{n}$ is a covering group of a symmetric or alternating group and completely classify non-trivial irreducible tensor products in characteristic $\neq 2$.

By definition there exists $z \in \widetilde{\mathrm{A}}_{n} \subseteq \widetilde{\mathrm{S}}_{n}$ with $z$ of order 2 and central in $\widetilde{\mathrm{S}}_{n}$ such that $\mathrm{S}_{n} \cong \widetilde{\mathrm{S}}_{n} /\langle z\rangle$ and $\mathrm{A}_{n} \cong \widetilde{\mathrm{A}}_{n} /\langle z\rangle$. Since $z$ is central of order 2, irreducible representations of $\widetilde{\mathrm{S}}_{n}$ and $\widetilde{\mathrm{A}}_{n}$ are of two types, depending on whether $z$ acts as 1 or -1 . Let $V$ be an irreducible representation of $\widetilde{\mathrm{S}}_{n}$ or $\widetilde{\mathrm{A}}_{n}$. If $z$ acts as 1 on $V$ then $V$ may be viewed also as a representation of $S_{n}$ or $A_{n}$ by factoring through $\langle z\rangle$ (and $V$ is irreducible also as an $\mathrm{S}_{n^{-}}$or $\mathrm{A}_{n}$-representation). On the other hand if $V$ is an (irreducible) representation of $\mathrm{S}_{n}$ or $\mathrm{A}_{n}$ then we may lift $V$ to an (irreducible) representation of $\widetilde{\mathrm{S}}_{n}$ or $\widetilde{\mathrm{A}}_{n}$ on which $z$ acts trivially. If on the other hand $z$ acts as -1 on $V$ then we say, when $p \neq 2$, that $V$ is a spin representation.

Thus, for $p \neq 2$, when considering tensor products $V \otimes W$ of two irreducible representations $V$ and $W$ of $\widetilde{\mathrm{S}}_{n}$ or $\widetilde{\mathrm{A}}_{n}$ three cases need to be considered: (i) neither $V$ nor $W$ is a spin representation, (ii) $V$ is not a spin representation, while $W$ is a spin representation and (iii) both $V$ and $W$ are spin representations. In case (i)

Received by the editors October 6, 2020, and, in revised form, March 26, 2021.

2020 Mathematics Subject Classification. Primary 20C30, 20C20, 20 C25.

The author was supported by the DFG grant MO 3377/1-1. This work was supported by: EPSRC grant number EP/R014604/1. 
$V \otimes W$ is irreducible as an $\widetilde{\mathrm{S}}_{n^{-}}$or $\widetilde{\mathrm{A}}_{n^{-}}$-representation if and only if it is irreducible as an $S_{n^{-}}$or $A_{n}$-representation, so this case is already covered by [5, 7, 19, 37, 38, 46, So only cases (ii) and (iii) will be considered in this paper. As can be seen from Theorems 1.1 and 1.2 irreducible tensor products of two spin representations only occur for $n$ small; however there exist infinite families of irreducible tensor products of a spin representation and a non-spin representation (see also [4,8,34 for partial results).

Note that if $p=2$ then $z$ acts trivially on any irreducible representation of $\widetilde{\mathrm{S}}_{n}$ or $\widetilde{\mathrm{A}}_{n}$. So in this case classifying irreducible tensor products of $\widetilde{\mathrm{S}}_{n}$ or $\widetilde{\mathrm{A}}_{n}$ is equivalent to classifying irreducible tensor products for $S_{n}$ or $A_{n}$. So this case will not be considered in this paper. For $S_{n}$ this problem has already been completely solved in $6,19,20,36$. For $\mathrm{A}_{n}$ partial results, including a complete analysis when neither $V$ nor $W$ is basic spin, can be found in 38 .

For $n=6$ or 7 , irreducible tensor products of representations of the triple covers can be easily classified looking at characters table using [25], so they will not be considered here.

It is well known (see for example [22]) that, in characteristic $p$, irreducible representations of symmetric groups are indexed by $p$-regular partitions, that is partitions with no part repeated $p$ or more times. Let $\mathscr{P}_{p}(n)$ be the set of $p$-regular partitions of $n$ and, given $\lambda \in \mathscr{P}_{p}(n)$, let $D^{\lambda}$ be the corresponding irreducible representation of $\mathrm{S}_{n}$. For any $\lambda \in \mathscr{P}_{p}(n)$ let $\lambda^{\mathrm{M}} \in \mathscr{P}_{p}(n)$ be the Mullineux dual of $\lambda$, that is the partition with $D^{\lambda^{M}} \cong D^{\lambda} \otimes \operatorname{sgn}$, where sgn is the sign representation of $\mathrm{S}_{n}$. A combinatorial description of the Mullineux bijection is known; see [9, 17, 41. For $p \geq 3$ it is also well known that $D^{\lambda} \downarrow_{\mathrm{A}_{n}}$ is irreducible if and only $\lambda \neq \lambda^{\mathrm{M}}$; see [16]. In this case we will write $E^{\lambda}$ for $D^{\lambda} \downarrow_{\mathrm{A}_{n}}$. Note that $E^{\lambda} \cong E^{\lambda^{\mathrm{M}}}$. On the other hand if $\lambda=\lambda^{\mathrm{M}}$ we have that $D^{\lambda} \downarrow_{\mathrm{A}_{n}} \cong E_{+}^{\lambda} \oplus E_{-}^{\lambda}$ with $E_{ \pm}^{\lambda}$ non-isomorphic irreducible representations of $A_{n}$. Further any irreducible representation of $A_{n}$ is either of the form $E^{\lambda}$ or of the form $E_{ \pm}^{\lambda}$ for some $\lambda \in \mathscr{P}_{p}(n)$. As mentioned above the modules $D^{\lambda}\left(\right.$ resp. $\left.E_{( \pm)}^{\lambda}\right)$ can also be viewed as representations of $\widetilde{\mathrm{S}}_{n}$ (resp. $\widetilde{\mathrm{A}}_{n}$ ).

In positive characteristic $p \geq 3$, irreducible spin representations of symmetric and alternating groups have been described in [12,13]. There it has been proved that if $\mathscr{R} \mathscr{P}_{p}(n)$ is the set of $p$-restricted $p$-strict partitions of $n$, that is partitions $\lambda$ with $1-\delta_{p \mid \lambda_{i}} \leq \lambda_{i}-\lambda_{i+1} \leq p-\delta_{p \mid n}$, then (pairs of) spin irreducible representations of $\widetilde{\mathrm{S}}_{n}$ or $\widetilde{\mathrm{A}}_{n}$ are indexed by elements of $\mathscr{R} \mathscr{P}_{p}(n)$ (here as in the following $\delta_{p \mid m}=1$ if $p \mid m$, while $\delta_{p \mid m}=0$ if $\left.p \nmid m\right)$. More in particular for any $\lambda \in \mathscr{R} \mathscr{P}_{p}(n)$ there either exists an irreducible spin representation $D(\lambda, 0)$ of $\widetilde{\mathrm{S}}_{n}$ or there exist two non-isomorphic representations $D(\lambda, \pm)$ of $\widetilde{\mathrm{S}}_{n}$. In either case, for $\varepsilon=0$ or \pm depending on $\lambda$, we have that $D(\lambda, \varepsilon) \cong D(\lambda,-\varepsilon) \otimes$ sgn, so that in the first case $D(\lambda, 0) \downarrow_{\tilde{A}_{n}} \cong E(\lambda,+) \oplus E(\lambda,-)$ with $E(\lambda, \pm)$ non-isomorphic irreducible spin representations of $\widetilde{\mathrm{A}}_{n}$, while in the second case $D(\lambda, \pm) \downarrow_{\tilde{\mathrm{A}}_{n}} \cong E(\lambda, 0)$ with $E(\lambda, 0)$ irreducible. Further again any spin irreducible representation of $\widetilde{S}_{n}$ or $\widetilde{A}_{n}$ is of one of these forms.

For $n \geq 1$ write $n=d p+e$ with $0 \leq e<p$. Define $\beta_{n}:=\left(p^{d}, e\right)$ if $e>0$ or $\beta_{n}:=\left(p^{d-1}, p-1,1\right)$ if $e=0$. Note that $\beta_{n}$ is a $p$-restricted $p$-strict partition of $n$. The irreducible spin representations indexed by $\beta_{n}$ are called basic spin modules and will play a special role in this paper. Such representations are the composition 
TABLE 1

\begin{tabular}{|c|c|c|c|c|c|}
\hline$G$ & $V$ & $W$ & $V \otimes W$ & $p$ & further assumptions \\
\hline$\widetilde{\mathrm{S}}_{6}$ & $D((3,2,1), \pm)$ & $D\left(\beta_{6}, \pm\right)$ & $D^{(3,2,1)}$ & $p \geq 7$ & \\
\hline$\widetilde{\mathrm{A}}_{5}$ & $E\left(\beta_{5},+\right)$ & $E\left(\beta_{5},-\right)$ & $E^{(4,1)}$ & $p \neq 5$ & \\
\hline$\widetilde{\mathrm{A}}_{6}$ & $E\left(\beta_{6},+\right)$ & $E\left(\beta_{6},-\right)$ & $E^{(5,1)}$ & $p=3$ & \\
\hline$\widetilde{\mathrm{A}}_{5}$ & $E_{ \pm}^{\left(3,1^{2}\right)}$ & $E\left(\beta_{5}, \pm\right)$ & $E((4,1), 0)$ & $p \neq 5$ & $\chi_{V} \chi_{W}(1,2,3,4,5)=1$ \\
\hline$\widetilde{\mathrm{A}}_{6}$ & $E_{ \pm}^{\left(4,1^{2}\right)}$ & $E\left(\beta_{6}, \pm\right)$ & $E((4,2), \pm)$ & $p=3$ & $\chi_{V} \chi_{W}(1, \widetilde{2,3,4}, 5)=1$ \\
\hline$\widetilde{\mathrm{A}}_{6}$ & $E_{+}^{\left(4,1^{2}\right)}$ & $E_{-}^{\left(4,1^{2}\right)}$ & $E^{(4,2)}$ & $p=3$ & \\
\hline$\widetilde{\mathrm{A}}_{9}$ & $E_{ \pm}^{\left(3^{3}\right)}$ & $E\left(\beta_{9}, \pm\right)$ & $E((5,3,1), \pm)$ & $p \geq 7$ & \\
\hline
\end{tabular}

factors of the reduction modulo $p$ of basic spin modules in characteristic 0 ; see 15, 45.

Given $\lambda \in \mathscr{P}_{p}(n)$ write $\lambda=\left(a_{1}^{b_{1}}, \ldots, a_{h}^{b_{h}}\right)$ with $a_{1}>\ldots>a_{h} \geq 1$ and $b_{i} \geq 1$. We say that $\lambda$ is JS if $a_{i}-a_{i+1}+b_{i}+b_{i+1} \equiv 0 \bmod p$ for $1 \leq i<h$. It has been proved (see [26, 27]) that $\lambda \in \mathscr{P}_{p}(n)$ is JS if and only if $D^{\lambda} \downarrow_{S_{n-1}}$ is irreducible. For any $a \geq 1$ let $a=b p+c$ with $1 \leq c \leq p$ and define $\operatorname{res}(a):=\min \{c-1, p-c\}$. For $\lambda \in \mathscr{R} \mathscr{P}_{p}(n)$ we say that $\lambda=\left(\lambda_{1}, \ldots, \lambda_{h}\right)$ is $\operatorname{JS}(0)$ if $\lambda_{h}=1$ and $\operatorname{res}\left(\lambda_{i}\right)=$ $\operatorname{res}\left(\lambda_{i+1}+1\right)$ for $1 \leq i<h$. In view of [12,43] it can be checked that $\lambda \in \mathscr{R} \mathscr{P}_{p}(n)$ is $\operatorname{JS}(0)$ if and only if $D(\lambda, \varepsilon) \downarrow_{\tilde{S}_{n-1}}$ and $E\left(\lambda, \varepsilon^{\prime}\right) \downarrow_{\tilde{A}_{n-1}}$ are both irreducible. An equivalent characterisation is also that $D(\lambda, 0) \downarrow_{\tilde{S}_{n-1}}$ is irreducible if $\lambda$ indexes only one spin representation of $\widetilde{\mathrm{S}}_{n}$ or that $E(\lambda, 0) \downarrow_{\tilde{A}_{n-1}}$ is irreducible if $\lambda$ indexes two spin representations of $\widetilde{S}_{n}$.

Before stating our main results we list here few irreducible tensor products of representations of $\widetilde{\mathrm{S}}_{n}$ or $\widetilde{\mathrm{A}}_{n}$, which will turn out to be exactly the exceptional irreducible tensor products of representations of $\widetilde{\mathrm{S}}_{n}$ or $\widetilde{\mathrm{A}}_{n}$; see Theorems 1.1 and 1.2 and their proof in $\$ 7$. As will be seen in the main theorems, any other irreducible tensor product is part of an infinite family of irreducible tensor products. In rows 4 and 5 of Table 1, $\chi_{V}$ is the character of $V, \chi_{W}$ the character of $W$ and $(1, \widetilde{2,3,4}, 5)$ the lift of order 5 of the 5 -cycle $(1,2,3,4,5)$.

In the next theorems, as well as in the remaining of the paper, if $\alpha$ and $\beta$ are partitions, let $\alpha+\beta:=\left(\alpha_{1}+\beta_{1}, \alpha_{2}+\beta_{2}, \ldots\right)$ and $\alpha \cup \beta$ be the partition obtained by rearranging the parts of $(\alpha, \beta)=\left(\alpha_{1}, \alpha_{2}, \ldots, \beta_{1}, \beta_{2}, \ldots\right)$. Further, for any partition $\alpha$, let $h(\alpha)$ be the number of non-zero parts of $\alpha$.

The next two theorems completely characterise irreducible tensor of representations of covering groups of symmetric and alternating groups respectively. Parts of the theorems (the classification of irreducible tensor products of two non-spin representations, the characteristic 0 case for $\tilde{S}_{n}$ and some reduction results for the other cases) can be recovered from the papers mentioned at the beginning of this section, but we still state the theorems in complete form.

Theorem 1.1. Let $p \geq 3$ and $V, W$ be irreducible $F \widetilde{\mathrm{S}}_{n}$-representations which are not 1-dimensional. Then $V \otimes W$ is irreducible if and only if one of the following holds up to exchange of $V$ and $W$ : 
(i) $n \not \equiv 0 \bmod p, V \in\left\{D^{(n-1,1)}, D^{(n-1,1)^{\mathrm{M}}}\right\}, W \cong D(\lambda, \pm)$ with $\lambda \in \mathscr{R} \mathscr{P}_{p}(n)$ a JS(0)-partition, in which case $V \otimes W \cong D(\nu, 0)$ where $\nu=(\lambda \backslash A) \cup B$ with $A$ is the bottom removable node of $\lambda$ and $B$ is the top addable node of $\lambda$,

(ii) $n \not \equiv 0, \pm 2 \bmod p$ is even, $V \cong D^{\lambda}$ where $\lambda \in \mathscr{P}_{p}(n)$ is a JS-partition with $\min \left\{h(\lambda), h\left(\lambda^{\mathrm{M}}\right)\right\}=2$, and $W$ is basic spin, in which case, assuming $h(\lambda)=2$, if $\lambda_{1} \neq \lambda_{2}$ then $V \otimes W \cong D\left(\beta_{\lambda_{1}}+\beta_{\lambda_{2}}, 0\right)$, while if $\lambda_{1}=\lambda_{2}$ then $V \otimes W \cong D\left(\beta_{n / 2+1} \cup \beta_{n / 2-1}, 0\right)$,

(iii) $V$ and $W$ are as in row 1 of Table 1 .

Theorem 1.2. Let $p \geq 3$ and $V, W$ be irreducible $F \widetilde{\mathrm{A}}_{n}$-representations which are not 1-dimensional. Then $V \otimes W$ is irreducible if and only if one of the following holds up to exchange of $V$ and $W$ :

(i) $n \not \equiv 0 \bmod p, V \cong E^{(n-1,1)}, W \cong E_{ \pm}^{\lambda}$ with $\lambda \in \mathscr{P}_{p}(n)$ a JS-partition satisfying $\lambda=\lambda^{\mathrm{M}}$, in which case $V \otimes W \cong E^{\nu}$ where $\nu=(\lambda \backslash A) \cup B$ with $A$ is the top removable node of $\lambda$ and $B$ is either of the two bottom addable nodes of $\lambda$,

(ii) $n \not \equiv 0 \bmod p, V \cong E^{(n-1,1)}, W \cong E(\lambda, \pm)$ with $\lambda \in \mathscr{R} \mathscr{P}_{p}(n)$ a $\operatorname{JS}(0)$ partition, in which case $V \otimes W \cong E(\nu, 0)$ where $\nu=(\lambda \backslash A) \cup B$ with $A$ is the bottom removable node of $\lambda$ and $B$ is the top addable node of $\lambda$,

(iii) $n \not \equiv 0, \pm 2 \bmod p$ is odd, $V \cong E^{\lambda}$ where $\lambda \in \mathscr{P}_{p}(n)$ is a JS-partition with $\min \left\{h(\lambda), h\left(\lambda^{\mathrm{M}}\right)\right\}=2$, and $W$ is basic spin, in which case, assuming $h(\lambda)=2$, if $\lambda_{1} \neq \lambda_{2}+p-2$ then $V \otimes W \cong E\left(\beta_{\lambda_{1}}+\beta_{\lambda_{2}}, 0\right)$, while if $\lambda_{1}=\lambda_{2}+p-2$ then $V \otimes W \cong E\left(\beta_{\lambda_{1}} \cup \beta_{\lambda_{2}}, 0\right)$,

(iv) $V$ and $W$ are as in rows $2-7$ of Table 1 .

Although in cases (ii) of Theorem 1.1 and (iii) of Theorem 1.2 we only describe for $V \otimes W$ if $h(\lambda)=2$, in the other case a description can be easily obtained, since $D^{\lambda^{\mathrm{M}}} \cong D^{\lambda} \otimes \operatorname{sgn}$ and $E^{\lambda^{\mathrm{M}}} \cong E^{\lambda}$.

In the next section we will introduce notation that will be used in the paper and state some well known/easy results. In $\$$ we will study endomorphism rings $\operatorname{End}_{F}(V)$ of general classes of modules $V$ of $\widetilde{\mathrm{S}}_{n}$ or $\widetilde{\mathrm{A}}_{n}$. In order to extend these results to some special classes of modules or at least obtain similar results in $\$ 5$, we will in $\$ 4$ study the structure of certain permutation modules. In both $\S \S 3$ and 5 we will often use branching results to obtain informations on $\operatorname{End}_{F}(V)$. In $\sqrt[6]{6}$ we will study tensor product with certain special classes of modules, using results on branching or known results in characteristic 0 and knowledge of decomposition matrices. Finally in 7 we will prove Theorems 1.1 and 1.2 .

\section{Notation AND BASIC RESUlts}

Throughout the paper $p$ will denote the characteristic of the field $F$ and we will assume that $p \neq 2$.

2.1. Covering groups. Let $\widetilde{\mathrm{S}}_{n}$ be any of the two double covers of $\mathrm{S}_{n}$ and $z$ be the non-trivial central element of $\widetilde{S}_{n}$ (which has order 2). There exists a short exact sequence

$$
1 \rightarrow\langle z\rangle \rightarrow \widetilde{\mathrm{S}}_{n} \stackrel{\pi}{\rightarrow} \mathrm{S}_{n} \rightarrow 1
$$

For any group $G \leq \mathrm{S}_{n}$ define $\widetilde{G}:=\pi^{-1} G \leq \widetilde{\mathrm{S}}_{n}$. In particular $\widetilde{\mathrm{A}}_{n}$ is the double cover of $\mathrm{A}_{n}$. Further for elements $g \in \mathrm{S}_{n}$ let $\widetilde{g} \in \widetilde{\mathrm{S}}_{n}$ be a (fixed) element in $\pi^{-1}\{g\}$, so 
that $\pi^{-1}\{g\}=\{\widetilde{g}, z \widetilde{g}\}$. If $g$ has odd order, one of the elements in $\pi^{-1}\{g\}$ has order $\operatorname{ord}(g)$, while the other has order $2 \operatorname{ord}(g)$. In this case choose $\widetilde{g}$ to have the same order as $g$.

As noted in $\oiint 1$, the irreducible representations of $F \widetilde{\mathrm{S}}_{n}$ (resp. $F \widetilde{\mathrm{A}}_{n}$ ) are given by the irreducible representations of $F \mathrm{~S}_{n}\left(\right.$ resp. $F \mathrm{~A}_{n}$ ), on which $z$ acts trivially, and the spin irreducible representations, on which $z$ acts as -1 .

Note that it does not matter which double cover of the symmetric group $S_{n}$ we consider, since the group algebras of the two double covers of $S_{n}$ are isomorphic; see [44, Note after Theorem 1.2] for the characteristic 0 case, the general case holding similarly.

2.2. Representations of symmetric and alternating groups. As noted in \$1 irreducible representations of $\mathrm{S}_{n}$ or $\mathrm{A}_{n}$ are indexed by elements of $\mathscr{P}_{p}(n)$, that is $p$-regular partitions of $n$. We write $\mathscr{P}_{p}^{A}(n)$ for the set of partitions $\lambda \in \mathscr{P}_{p}(n)$ with $\lambda=\lambda^{\mathrm{M}}$, that is partitions $\lambda$ for which $D^{\lambda} \downarrow_{\mathrm{A}_{n}}$ splits.

Given a partition $\lambda \in \mathscr{P}_{p}(n)$ define normal, good, conormal and cogood nodes of $\lambda$ as in [30, §11.1]. It can be easily seen from the definition that $\lambda$ is JS if and only if it has only one normal node.

If $(a, b)$ is a node, let $(b-a) \bmod p$ be the residue of $(a, b)$. For any partition $\lambda$ let the content of $\lambda$ be the tuple $\left(a_{0}, \ldots, a_{p-1}\right)$, where $a_{i}$ is the number of nodes of $\lambda$ of residue $i$ for each $0 \leq i<p$. It is well known that if $\lambda, \mu \in \mathscr{P}_{p}(n)$, then $D^{\lambda}$ and $D^{\mu}$ are in the same block if and only if $\lambda$ and $\mu$ have the same content; see [24, 2.7.41, 6.1.21] and [22, §11], so that we may speak of content of a block or of a block with a certain content (which is unique if such a block exists). Let $V$ be an $F S_{n}$-module in a block $B$ with content $\left(a_{0}, \ldots, a_{p-1}\right)$. For any residue $i$, we define $e_{i} V$ to be the projection of $V \downarrow_{\mathrm{S}_{n-1}}$ to the block with content $\left(a_{0}, \ldots, a_{i-1}, a_{i}-1, a_{i+1}, \ldots, a_{p-1}\right)$ and $f_{i} V$ to be the projection of $V \uparrow S_{n+1}$ to the block with content $\left(a_{0}, \ldots, a_{i-1}, a_{i}+1, a_{i+1}, \ldots, a_{p-1}\right)$. We then extend the definition of $e_{i} V$ and $f_{i} V$ to arbitrary $F S_{n}$-modules additively to obtain functors

$e_{i}: \quad \bmod F S_{n} \rightarrow \bmod F S_{n-1}, \quad f_{i}: \quad \bmod F S_{n} \rightarrow \bmod F S_{n+1}$.

More generally, for any $r \geq 1$ let

$e_{i}^{(r)}: \bmod F \mathrm{~S}_{n} \rightarrow \bmod F \mathrm{~S}_{n-r}, \quad f_{i}^{(r)}: \bmod F \mathrm{~S}_{n} \rightarrow \bmod F \mathrm{~S}_{n+r}$,

be the divided power functors; see [30, §11.2]. The following is well-known; see for example [30, Lemma 8.2.2(ii), Theorems 8.3.2(i), 11.2.7, 11.2.8]:

Lemma 2.1. For any residue $i$ and any $r \geq 1$, the functors $e_{i}^{(r)}$ and $f_{i}^{(r)}$ are biadjoint and commute with duality. Further, for any $F \mathrm{~S}_{n}$-module $V$ we have

$$
V \downarrow_{\mathrm{S}_{n-1}} \cong e_{0} V \oplus \ldots \oplus e_{p-1} V \quad \text { and } \quad V \uparrow^{\mathrm{S}_{n+1}} \cong f_{0} V \oplus \ldots \oplus f_{p-1} V .
$$

For any partition $\lambda \in \mathscr{P}_{p}(n)$ and any residue $i$, let $\varepsilon_{i}(\lambda)$ be its number of $i$-normal nodes and $\varphi_{i}(\lambda)$ be its number of $i$-conormal nodes. If $\varepsilon_{i}(\lambda)>0$ let $\widetilde{e}_{i} \lambda \in \mathscr{P}_{p}(n-1)$ be the partition obtained from $\lambda$ by removing the bottom $i$-normal node, while if $\varphi_{i}(\lambda)>0$ let $\tilde{f}_{i} \lambda \in \mathscr{P}_{p}(n+1)$ be the partition obtained from $\lambda$ by adding the top $i$-conormal node (see [30, §11.1]). For $r \geq 2$ define

$$
e_{i}^{r} V:=\underbrace{e_{i} \cdots e_{i}}_{r \text { times }} V
$$


and define similarly $f_{i}^{r} V, \tilde{e}_{i}^{r} \lambda$ and $\tilde{f}_{i}^{r} \lambda$ (the last two are only defined if $\varepsilon_{i}(\lambda) \geq r$ or $\varphi_{i}(\lambda) \geq r$ respectively).

The following two results hold by [10, Theorems E(iv), E'(iv)], [30, Theorems 11.2.10, 11.2.11] and [29, Theorem 1.4].

Lemma 2.2. Let $\lambda \in \mathscr{P}_{p}(n)$. Then for any residue $i$ and any $r \geq 1$ :

(i) $e_{i}^{r} D^{\lambda} \cong\left(e_{i}^{(r)} D^{\lambda}\right)^{\oplus r !}$;

(ii) $e_{i}^{(r)} D^{\lambda} \neq 0$ if and only if $r \leq \varepsilon_{i}(\lambda)$, in which case $e_{i}^{(r)} D^{\lambda}$ is a self-dual indecomposable module with socle and head both isomorphic to $D^{\widetilde{e}_{i}^{r} \lambda}$.

(iii) $\left[e_{i}^{(r)} D^{\lambda}: D^{\widetilde{e}_{i}^{r} \lambda}\right]=\left(\begin{array}{c}\varepsilon_{i}(\lambda) \\ r\end{array}\right)=\operatorname{dim} \operatorname{End}_{\mathrm{S}_{n-r}}\left(e_{i}^{(r)} D^{\lambda}\right)$;

(iv) if $D^{\mu}$ is a composition factor of $e_{i}^{(r)} D^{\lambda}$ then $\varepsilon_{i}(\mu) \leq \varepsilon_{i}(\lambda)-r$, with equality holding if and only if $\mu=\widetilde{e}_{i}^{r} \lambda$;

(v) $\operatorname{dim} \operatorname{End}_{\mathrm{S}_{n-1}}\left(D^{\lambda} \downarrow_{\mathrm{S}_{n-1}}\right)=\sum_{j \in I} \varepsilon_{j}(\lambda)$.

(vi) Let $A$ be a removable node of $\lambda$ such that $\lambda_{A}$ is p-regular. Then $D^{\lambda_{A}}$ is a composition factor of $e_{i} D^{\lambda}$ if and only if $A$ is $i$-normal, in which case $\left[e_{i} D^{\lambda}: D^{\lambda_{A}}\right]$ is one more than the number of $i$-normal nodes for $\lambda$ above $A$.

Lemma 2.3. Let $\lambda \in \mathscr{P}_{p}(n)$. Then for any residue $i$ and any $r \geq 1$ :

(i) $f_{i}^{r} D^{\lambda} \cong\left(f_{i}^{(r)} D^{\lambda}\right)^{\oplus r !}$;

(ii) $f_{i}^{(r)} D^{\lambda} \neq 0$ if and only if $r \leq \varphi_{i}(\lambda)$, in which case $f_{i}^{(r)} D^{\lambda}$ is a self-dual indecomposable module with socle and head both isomorphic to $D^{\widetilde{f}_{i}^{r} \lambda}$.

(iii) $\left[f_{i}^{(r)} D^{\lambda}: D^{\widetilde{f}_{i}^{r} \lambda}\right]=\left(\begin{array}{c}\varphi_{i}(\lambda) \\ r\end{array}\right)=\operatorname{dim} \operatorname{End}_{S_{n+r}}\left(f_{i}^{(r)} D^{\lambda}\right)$;

(iv) if $D^{\mu}$ is a composition factor of $f_{i}^{(r)} D^{\lambda}$ then $\varphi_{i}(\mu) \leq \varphi_{i}(\lambda)-r$, with equality holding if and only if $\mu=\widetilde{f}_{i}^{r} \lambda$.

(v) $\operatorname{dim} \operatorname{End}_{\mathrm{S}_{n+1}}\left(D^{\lambda \uparrow \mathrm{S}_{n+1}}\right)=\sum_{j \in I} \varphi_{j}(\lambda)$.

(vi) Let $B$ be an addable node for $\lambda$ such that $\lambda^{B}$ is p-regular. Then $D^{\lambda^{B}}$ is a composition factor of $f_{i} D^{\lambda}$ if and only if $B$ is $i$-conormal, in which case $\left[f_{i} D^{\lambda}: D^{\lambda^{B}}\right]$ is one more than the number of $i$-conormal nodes for $\lambda$ below $B$.

Lemma 2.4 compares the functors $e_{i} e_{j}$ and $e_{j} e_{i}$ for different residues $i$ and $j$.

Lemma 2.4 ([36, Lemma 4.8]). Let $\lambda \vdash n$ be $p$-regular. For $i \neq j$ we have that

$$
\operatorname{dim} \operatorname{Hom}_{\mathrm{S}_{n-2}}\left(e_{j} e_{i} D^{\lambda}, e_{i} e_{j} D^{\lambda}\right) \geq \varepsilon_{i}(\lambda) \varepsilon_{j}(\lambda) .
$$

When considering (co)good or (co)normal nodes and the Mullineux map we have the following result:

Lemma 2.5 ([28, Theorem 4.7]). For any partition $\lambda$ and for any residue $i$,

$$
\varepsilon_{i}(\lambda)=\varepsilon_{-i}\left(\lambda^{\mathrm{M}}\right) \quad \text { and } \quad \varphi_{i}(\lambda)=\varphi_{-i}\left(\lambda^{\mathrm{M}}\right) .
$$

If $\varepsilon_{i}(\lambda)>0$ then $\widetilde{e}_{i}(\lambda)^{\mathrm{M}}=\widetilde{e}_{-i}\left(\lambda^{\mathrm{M}}\right)$, while if $\varphi_{i}(\lambda)>0$ then $\widetilde{f}_{i}\left(\lambda^{\mathrm{M}}\right)=\widetilde{f}_{-i}\left(\lambda^{\mathrm{M}}\right)$.

2.3. Spin representations. As noted in $\$ 1$ spin irreducible representations of $\widetilde{\mathrm{S}}_{n}$ and $\widetilde{\mathrm{A}}_{n}$ in characteristic $p \neq 0$ are indexed by elements of $\mathscr{R} \mathscr{P}_{p}(n)$, that is $p$ restricted $p$-strict partitions of $n$. In characteristic 0 they are instead indexed by $\mathscr{R} \mathscr{P}_{0}(n)$, the set of partitions in distinct parts; see for example [44. In view of Lemma 2.17, the labeling in characteristic 0 exactly corresponds to the labeling in 
characteristic $>n$, as is the case for the non-spin case (see for example [22]). For $\lambda \in \mathscr{R} \mathscr{P}_{p}(n)$ remember that $h(\lambda)$ is the number of parts of $\lambda$ and define $h_{p^{\prime}}(\lambda)$ to be the number of parts of $\lambda$ which are not divisible by $p$. If $n-h_{p^{\prime}}(\lambda)$ is even let $a(\lambda):=0$, while if $n-h_{p^{\prime}}(\lambda)$ is odd let $a(\lambda):=1$. In [12,13, it has been proved that if $a(\lambda)=0$ then $\lambda$ indexes one spin irreducible representation of $\widetilde{\mathrm{S}}_{n}$ and two of $\widetilde{\mathrm{A}}_{n}$, while if $a(\lambda)=1$ then $\lambda$ indexes two spin irreducible representations of $\widetilde{\mathrm{S}}_{n}$ and one of $\widetilde{\mathrm{A}}_{n}$. So

$$
\begin{aligned}
& \left\{D(\lambda, 0) \mid \lambda \in \mathscr{R} \mathscr{P}_{p}(n) \text { with } a(\lambda)=0\right\} \\
& \cup\left\{D(\lambda,+), D(\lambda,-) \mid \lambda \in \mathscr{R} \mathscr{P}_{p}(n) \text { with } a(\lambda)=1\right\}
\end{aligned}
$$

is a complete set of spin irreducible $F \widetilde{\mathrm{S}}_{n}$-representations up to isomorphism and

$$
\begin{aligned}
& \left\{E(\lambda,+), E(\lambda,-) \mid \lambda \in \mathscr{R} \mathscr{P}_{p}(n) \text { with } a(\lambda)=0\right\} \\
& \cup\left\{E(\lambda, 0) \mid \lambda \in \mathscr{R} \mathscr{P}_{p}(n) \text { with } a(\lambda)=1\right\}
\end{aligned}
$$

is a complete set of spin irreducible $F \widetilde{\mathrm{A}}_{n}$-representations up to isomorphism.

When $a(\lambda)=1$ it is often easier to work with $D(\lambda,+) \oplus D(\lambda,-)$ instead of working with $D(\lambda,+)$ and $D(\lambda,-)$ separately. For this reason we define the irreducible supermodule

$$
D(\lambda):= \begin{cases}D(\lambda, 0), & a(\lambda)=0 \\ D(\lambda,+) \oplus D(\lambda,-), & a(\lambda)=1 .\end{cases}
$$

Similarly we define $E(\lambda)$. We say that $D(\lambda)$ is of type M if $a(\lambda)=0$ or of type Q if $a(\lambda)=1$. Note that $\operatorname{dim} \operatorname{End}_{\widetilde{\mathrm{S}}_{n}}(D(\lambda))=1+a(\lambda)$, since if $a(\lambda)=1$ then $D(\lambda)$ is the direct sum of two non-isomorphic simple modules.

When considering spin modules of $\mathrm{S}_{n}$ in characteristic 0 we will also write $S(\lambda)$ for $D(\lambda)$ and similarly $S(\lambda, 0)$ or $S(\lambda, \pm)$ for $D(\lambda, 0)$ or $D(\lambda, \pm)$.

Given supermodules $V$ of $\widetilde{\mathrm{S}}_{\mu}$ and $W$ of $\widetilde{\mathrm{S}}_{\nu}$ (with $\mu, \nu$ compositions) we can consider their "outer" tensor product $V \otimes W$ as a supermodule of $\widetilde{\mathrm{S}}_{\mu, \nu}$. Outer tensor products of supermodules are not always simple as supermodules (see for example [12, $\S 2-\mathrm{b}])$. If $V$ and $W$ are irreducible supermodules, then there exists an irreducible supermodule $M \circledast N$ such that:

- if both $V$ and $W$ are of type $\mathrm{M}$ then $V \otimes W \cong V \circledast W$ is of type $\mathrm{M}$,

- if one $V$ and $W$ is of type $\mathrm{M}$ and the other of type Q then $V \otimes W \cong V \circledast W$ is of type $\mathrm{Q}$,

- if both $V$ and $W$ are of type Q then $V \otimes W \cong(V \circledast W)^{\oplus 2}$ with $V \circledast W$ of type $\mathrm{M}$.

For partitions $\lambda^{j} \in \mathscr{R} \mathscr{P}_{p}\left(n_{j}\right)$, this can then be extended to define simple supermodules $D\left(\lambda^{1}\right) \circledast \cdots \circledast D\left(\lambda^{h}\right)$. We will write $D\left(\lambda^{1}, \ldots, \lambda^{h}\right)$ for $D\left(\lambda^{1}\right) \circledast \cdots \circledast D\left(\lambda^{h}\right)$ and $D\left(\lambda^{1}, \ldots, \lambda^{h}, 0\right)$ or $D\left(\lambda^{1}, \ldots, \lambda^{h}, \pm\right)$ for its simple components (as module).

For supermodules $V$ and $W$, we write $V \simeq W$ if there exists an even isomorphism $V \rightarrow W$ (see [12, §2-b] and [13, §2]). In particular if $V \simeq W$ then $V \cong W$ as modules.

There are branching rules for spin irreducible supermodules which are similar to branching rules for irreducible representations of symmetric groups. Before stating them, we need to define some combinatorial notions. We start by defining residues of nodes. The residue of the node $(a, b)$ is given by $\operatorname{res}(b)$, where res $(b)$ is defined as in 91 . So the residue of any node is an integer $i$ with $0 \leq i \leq \ell$, where $\ell=\ell_{p}=$ 
$(p-1) / 2$, and on any row residues are given by

$$
0,1, \ldots, \ell-1, \ell, \ell-1, \ldots, 1,0,0,1, \ldots, \ell-1, \ell, \ell-1, \ldots, 1,0, \ldots
$$

Again define the content of a partition $\lambda$ to be $\left(a_{0}, \ldots, a_{\ell}\right)$ if for every $0 \leq i \leq \ell$ we have that $\lambda$ has $a_{i}$ nodes of residue $i$. Normal nodes (and conormal, good and cogood nodes) can be defined also for $p$-restricted $p$-strict partitions; see for example [12, $\S 9$-a]. Let $\lambda \in \mathscr{R} \mathscr{P}_{p}(n)$. For $0 \leq i \leq(p-1) / 2$ let $\varepsilon_{i}(\lambda)$ be the number of $i$-normal nodes of $\lambda$ and $\varphi_{i}(\lambda)$ be the number of $i$-conormal nodes of $\lambda$. If $\varepsilon_{i}(\lambda)>0$ we write $\widetilde{e}_{i} \lambda$ for the partition obtained from $\lambda$ by removing the $i$-good node of $\lambda$. Similarly, if $\varphi_{i}(\lambda)>0$ we write $\tilde{f}_{i} \lambda$ for the partition obtained from $\lambda$ by adding the $i$-cogood node of $\lambda$. We say that $\lambda \in \mathscr{R} \mathscr{P}_{p}(n)$ is JS if it has only one normal node. As will be seen for example in Lemmas 2.7 and 2.11, the residue of the normal node will play an important role (in particular it is important if the unique normal node has residue 0 or not). If $\lambda$ is JS and its normal node has residue $i$ we say that $\lambda$ is $\operatorname{JS}(i)$ (or write $\lambda \in \operatorname{JS}(i)$ ). For $i=0$ a combinatorial description of $\operatorname{JS}(0)$ partitions has been given in $\$ 1$. By definition we easily have that

Lemma 2.6. Let $\lambda \in \mathscr{R} \mathscr{P}_{p}(n)$ and $0 \leq i \leq(p-1) / 2$.

- if $\varepsilon_{i}(\lambda)>0$ then $\varphi_{i}\left(\widetilde{e}_{i} \lambda\right)>0$ and $\widetilde{f}_{i} \widetilde{e}_{i} \lambda=\lambda$. Further if $i=0$ then $a\left(\widetilde{e}_{i} \lambda\right)=a(\lambda)$, while if $i>0$ then $a\left(\widetilde{e}_{i} \lambda\right)=1-a(\lambda)$;

- if $\varphi_{i}(\lambda)>0$ then $\varepsilon_{i}\left(\widetilde{f}_{i} \lambda\right)>0$ and $\widetilde{e}_{i} \widetilde{f}_{i} \lambda=\lambda$. Further if $i=0$ then $a\left(\widetilde{f}_{i} \lambda\right)=a(\lambda)$, while if $i>0$ then $a\left(\widetilde{f}_{i} \lambda\right)=1-a(\lambda)$.

It can be checked that $D(\lambda, \delta)$ and $D(\mu, \varepsilon)$ are in the same block if and only if $\lambda$ and $\mu$ have the same content (unless possibly if $\lambda=\mu$ is a $p$-bar core, in which case the blocks have weight 0); see [30, (22.7), 22.3.20] and the definition of $p$-bar core just before [30, (22.7)]. If $M$ is a spin module of $\widetilde{\mathrm{S}}_{n}$ contained in the block(s) with content $\left(a_{0}, \ldots, a_{\ell}\right)$ and $i$ is a residue, let $\operatorname{Res}_{i} M$ be the block(s) component(s) of $M \downarrow_{\widetilde{\mathrm{S}}_{n-1}}$ corresponding to the blocks with content $\left(a_{0}, \ldots, a_{i-1}, a_{i}-1, a_{i+1}, \ldots, a_{\ell}\right)$. Define similarly $\operatorname{Ind}_{i} M$ as the block(s) component(s) of $M \uparrow^{\widetilde{S}_{n+1}}$ corresponding to the blocks with content $\left(a_{0}, \ldots, a_{i-1}, a_{i}+1, a_{i+1}, \ldots, a_{\ell}\right)$. This can then be extended to arbitrary spin modules. Often the modules $\operatorname{Res}_{i} D(\lambda)$ and $\operatorname{Ind}_{i} D(\lambda)$ are not indecomposable as supermodules. However there exist modules $e_{i} D(\lambda)$ and $f_{i} D(\lambda)$ such that the following; see [12, Theorems 9.13, 9.14] and [32, Theorem A]:

Lemma 2.7. Let $\lambda \in \mathscr{R} \mathscr{P}_{p}(n)$ and $0 \leq i \leq \ell$. Then:

(i) $\operatorname{Res}_{i} D(\lambda) \cong\left(e_{i} D(\lambda)\right)^{\oplus 1+a(\lambda) \delta_{i}>0}$;

(ii) $D(\lambda) \downarrow_{\widetilde{S}_{n-1}} \cong e_{0} D(\lambda) \oplus \bigoplus_{j=1}^{\ell}\left(e_{j} D(\lambda)\right)^{\oplus 1+a(\lambda)}$;

(iii) $e_{i} D(\lambda) \stackrel{\neq}{\neq}$ if and only if $\varepsilon_{i}(\lambda)>0$, in which case $e_{i} D(\lambda)$ is a selfdual indecomposable supermodule with socle and head both isomorphic to $D\left(\widetilde{e}_{i} \lambda\right)$;

(iv) $\left[e_{i} D(\lambda): D\left(\widetilde{e}_{i} \lambda\right)\right]=\varepsilon_{i}(\lambda)$;

(v) if $D(\mu)$ is a composition factor of $e_{i} D(\lambda)$ then $\varepsilon_{i}(\mu) \leq \varepsilon_{i}(\lambda)-1$, with equality holding if and only if $\mu=\widetilde{e}_{i} \lambda$;

(vi) $\operatorname{End}_{\widetilde{\mathrm{S}}_{n-1}}\left(e_{i} D(\lambda)\right) \simeq \operatorname{End}_{\widetilde{\mathrm{S}}_{n-1}}\left(D\left(\widetilde{e}_{i} \lambda\right)\right)^{\oplus \varepsilon_{i}(\lambda)}$;

(vii) $\operatorname{Hom}_{\widetilde{\mathrm{S}}_{n-1}}\left(e_{i} D(\lambda), e_{i} D(\nu)\right)=0$ if $\nu \in \mathscr{R} \mathscr{P}_{p}(n)$ with $\nu \neq \lambda$;

(viii) if $A$ is an $i$-normal node of $\lambda$ such that $\lambda \backslash A \in \mathscr{R} \mathscr{P}_{p}(n-1)$, then $D(\lambda \backslash A)$ is a composition factor of $e_{i} D(\lambda)$. 
Lemma 2.8. Let $\lambda \in \mathscr{R} \mathscr{P}_{p}(n), 0 \leq i \leq(p-1) / 2$. Then:

(i) $\operatorname{Ind}_{i} D(\lambda) \cong\left(f_{i} D(\lambda)\right)^{\oplus 1+a(\lambda) \delta_{i}>0}$;

(ii) $D(\lambda) \uparrow^{\widetilde{S}_{n+1}} \cong f_{0} D(\lambda) \oplus \bigoplus_{j=1}^{\ell}\left(f_{j} D(\lambda)\right)^{\oplus 1+a(\lambda)}$;

(iii) $f_{i} D(\lambda) \neq 0$ if and only if $\varphi_{i}(\lambda)>0$, in which case $f_{i} D(\lambda)$ is a selfdual indecomposable supermodule with socle and head both isomorphic to $D\left(\widetilde{f}_{i} \lambda\right)$;

(iv) $\left[f_{i} D(\lambda): D\left(\widetilde{f}_{i} \lambda\right)\right]=\varphi_{i}(\lambda)$;

(v) if $D(\mu)$ is a composition factor of $f_{i} D(\lambda)$ then $\varphi_{i}(\mu) \leq \varphi_{i}(\lambda)-1$, with equality holding if and only if $\mu=\widetilde{f}_{i} \lambda$;

(vi) $\operatorname{End}_{\widetilde{\mathrm{S}}_{n+1}}\left(f_{i} D(\lambda)\right) \simeq\left(\operatorname{End}_{\widetilde{\mathrm{S}}_{n+1}}\left(D\left(\widetilde{f}_{i} \lambda\right)\right)\right)^{\oplus \varphi_{i}(\lambda)}$;

(vii) $\operatorname{Hom}_{\widetilde{\mathrm{S}}_{n+1}}\left(f_{i} D(\lambda), f_{i} D(\nu)\right)=0$ if $\nu \in \mathscr{R} \mathscr{P}_{p}(n)$ with $\nu \neq \lambda$;

(viii) if $B$ is an $i$-conormal node of $\lambda$ such that $\lambda \cup B \in \mathscr{R} \mathscr{P}_{p}(n+1)$, then $D(\lambda \cup B)$ is a composition factor of $f_{i} D(\lambda)$.

When considering restrictions to $\widetilde{\mathrm{S}}_{n-r}$ we have that there exists divided power modules $e_{i}^{(r)} D(\lambda)$ with $e_{i}^{(1)} D(\lambda) \cong e_{i} D(\lambda)$ such that the following holds; see 30 , Lemma 22.3.15] for the first part and use Lemma 2.7 to obtain the other two (there also exists divided power $F \widetilde{\mathrm{S}}_{n+r}$-modules $f_{i}^{(r)} D(\lambda)$ with corresponding properties, though these will not be needed in this paper). Again define $\operatorname{Res}_{i}^{r} V$ and $\tilde{e}_{i}^{r} \lambda$ similarly to what had been done for the non-spin case.

Lemma 2.9. Let $\lambda \in \mathscr{R} \mathscr{P}_{p}(n), 0 \leq i \leq(p-1) / 2$. Then:

(i) $\operatorname{Res}_{i}^{r} D(\lambda) \cong\left(e_{i}^{(r)} D(\lambda)\right)^{\oplus 2^{\delta_{i>0}\lfloor(r+a(\lambda)) / 2\rfloor} r \text { ! }}$

(ii) $e_{i}^{(r)} D(\lambda) \neq 0$ if and only if $\varepsilon_{i}(\lambda) \geq r$;

(iii) $\left[e_{i}^{(r)} D(\lambda): D\left(\widetilde{e}_{i}^{r} \lambda\right)\right]=\left(\begin{array}{c}\varepsilon_{i}(\lambda) \\ r\end{array}\right)$.

Further by [30, Lemma 19.1.1, Theorems 22.2.2, 22.2.3]:

Lemma 2.10. The functors $e_{i}$ and $f_{i}$ are biadjoint and commute with duality.

Comparing the number of normal and conormal nodes, we obtain Lemma 2.11. which holds by Lemmas 2.6, 2.7 and 2.8,

Lemma 2.11. Let $\lambda \in \mathscr{R} \mathscr{P}_{p}(n)$. Then

$$
\begin{aligned}
\operatorname{dim} \operatorname{End}_{\widetilde{\mathrm{S}}_{n-1}}\left(D(\lambda) \downarrow_{\widetilde{\mathrm{S}}_{n-1}}\right) & =\left(\varepsilon_{0}(\lambda)+2 \sum_{i \geq 1} \varepsilon_{i}(\lambda)\right) \operatorname{dim} \operatorname{End}_{\widetilde{\mathrm{S}}_{n}}(D(\lambda)), \\
\operatorname{dim} \operatorname{End}_{\widetilde{\mathrm{S}}_{n+1}}\left(D(\lambda) \uparrow \widetilde{\boldsymbol{S}}_{n+1}\right) & =\left(\varphi_{0}(\lambda)+2 \sum_{i \geq 1} \varphi_{i}(\lambda)\right) \operatorname{dim} \operatorname{End}_{\widetilde{\mathrm{S}}_{n}}(D(\lambda)) .
\end{aligned}
$$

Further, by the same lemmas, the following holds about the module $D(\lambda) \downarrow_{\widetilde{\mathrm{s}}_{n-1}} \uparrow^{\widetilde{\mathrm{S}}_{n}}$ :

Lemma 2.12. Let $\lambda \in \mathscr{R} \mathscr{P}_{p}(n)$. Then

$$
\left[\operatorname{Ind}_{i} \operatorname{Res}_{i} D(\lambda): D(\lambda)\right]=\varepsilon_{i}(\lambda)\left(\varphi_{i}(\lambda)+1\right)\left(1+\delta_{i>0}\right) .
$$

In particular

$$
\left[D(\lambda) \otimes M_{1}: D(\lambda)\right]=\varepsilon_{0}(\lambda)\left(\varphi_{0}(\lambda)+1\right)+2 \sum_{i \geq 1} \varepsilon_{i}(\lambda)\left(\varphi_{i}(\lambda)+1\right) .
$$


By Mackey induction-reduction theorem we have that $M \uparrow^{\widetilde{\mathrm{S}}_{n+1}} \downarrow \widetilde{\mathrm{S}}_{n} \cong M \oplus M \downarrow^{\widetilde{\mathrm{S}}_{n-1} \uparrow \widetilde{\mathrm{S}}_{n}}$ for any module $M$ of $F \widetilde{\mathrm{S}}_{n}$. The next two lemmas then follow (for the first one use also Lemma 2.11):

Lemma 2.13. Let $\lambda \in \mathscr{R} \mathscr{P}_{p}(n)$. Then

$$
\varepsilon_{0}(\lambda)+2 \sum_{i \geq 1} \varepsilon_{i}(\lambda)+1=\varphi_{0}(\lambda)+2 \sum_{i \geq 1} \varphi_{i}(\lambda) .
$$

In particular $\varepsilon_{0}(\lambda)+\varphi_{0}(\lambda)$ is odd.

Lemma 2.14. If $i \neq j$ and $A$ is any spin module of $\widetilde{\mathrm{S}}_{n}$ then $\operatorname{Ind}_{j} \operatorname{Res}_{i} M \cong$ $\operatorname{Res}_{i} \operatorname{Ind}_{j} M$.

The next results consider normal nodes of different residues.

Lemma 2.15. Let $\lambda \in \mathscr{R} \mathscr{P}_{p}(n)$. If $i \neq j$ and $\varepsilon_{i}(\lambda), \varepsilon_{j}(\lambda)>0$ then $\operatorname{End}_{\widetilde{\mathrm{S}}_{n-2}}\left(e_{i} D\left(\widetilde{e}_{j} \lambda\right)\right.$, $\left.e_{j} D\left(\widetilde{e}_{i} \lambda\right)\right) \neq 0$.

Proof. Using Lemmas 2.7, 2.8 and 2.14 we have that there exists $c>0$ such that

$$
\begin{aligned}
\operatorname{dim} \operatorname{End}_{\widetilde{\mathrm{S}}_{n-2}}\left(e_{i} D\left(\widetilde{e}_{j} \lambda\right), e_{j} D\left(\widetilde{e}_{i} \lambda\right)\right) & =c \operatorname{dim} \operatorname{End}_{\widetilde{\mathrm{S}}_{n-1}}\left(\operatorname{Ind}_{j} \operatorname{Res}_{i} D\left(\widetilde{e}_{j} \lambda\right), D\left(\widetilde{e}_{i} \lambda\right)\right) \\
& =c \operatorname{dim} \operatorname{End}_{\widetilde{\mathrm{S}}_{n-1}}\left(\operatorname{Res}_{i} \operatorname{Ind}_{j} D\left(\widetilde{e}_{j} \lambda\right), D\left(\widetilde{e}_{i} \lambda\right)\right) \\
& =c \operatorname{End}_{\widetilde{S}_{n}}\left(\operatorname{Ind}_{j} D\left(\widetilde{e}_{j} \lambda\right), \operatorname{Ind}_{i} D\left(\widetilde{e}_{i} \lambda\right)\right)
\end{aligned}
$$

from which the lemma follows, since both $\operatorname{Ind}_{j} D\left(\widetilde{e}_{j} \lambda\right)$ and $\operatorname{Ind}_{i} D\left(\widetilde{e}_{i} \lambda\right)$ contain $D(\lambda)$ in their head and socle by Lemmas 2.6 and 2.8 .

Lemma 2.16. If $\lambda \in \mathscr{R} \mathscr{P}_{p}(n)$ and $\varepsilon_{i}(\lambda)>0$ then $\varepsilon_{j}\left(\widetilde{e}_{i} \lambda\right) \geq \varepsilon_{j}(\lambda)$ for $j \neq i$.

Proof. We may assume that $\varepsilon_{j}(\lambda)>0$. Then from Lemmas 2.6, 2.8 and 2.14,

$$
0 \neq \operatorname{Res}_{i}^{\varepsilon_{i}(\lambda)} D(\lambda) \subseteq \operatorname{Res}_{i}^{\varepsilon_{i}(\lambda)} \operatorname{Ind}_{j} D\left(\widetilde{e}_{j} \lambda\right) \cong \operatorname{Ind}_{j} \operatorname{Res}_{i}^{\varepsilon_{i}(\lambda)} D\left(\widetilde{e}_{j} \lambda\right) .
$$

In particular $\operatorname{Res}_{i}^{\varepsilon_{i}(\lambda)} D\left(\widetilde{e}_{j} \lambda\right) \neq 0$ from which the lemma follows by Lemma 2.9.

2.4. Reduction modulo $p$. We now consider some results about reduction modulo $p$ of spin representations in characteristic 0 . If $\mu \in \mathscr{R} \mathscr{P}_{0}(n)$, let $\mu^{R} \in \mathscr{R} \mathscr{P}_{p}(n)$ be as defined in [15]. Given two partitions $\alpha, \beta$, write $\alpha \triangleleft \beta$ if $\alpha$ is smaller than $\beta$ in the dominance order. The main known result is the following; see 13, Theorem 10.8], [14, Theorem 10.4], and [15, Theorem 4.4]:

Lemma 2.17. Let $\mu \in \mathscr{R} \mathscr{P}_{0}(n)$ and $\nu \in \mathscr{R} \mathscr{P}_{p}(n)$. If $[S(\mu): D(\nu)]>0$ then $\nu \unlhd \mu^{R}$. Further

$$
\left[S(\mu): D\left(\mu^{R}\right)\right]=2^{\left(h(\mu)-h_{p^{\prime}}(\mu)+a(\mu)-a\left(\mu^{R}\right)\right) / 2} .
$$

Let $n=a p+b$ with $0 \leq b<p$. The spin irreducible representations of $\widetilde{\mathrm{S}}_{n}$ and $\widetilde{\mathrm{A}}_{n}$ indexed by the partition

$$
\beta_{n}:= \begin{cases}\left(p^{a}, b\right), & b \neq 0 \\ \left(p^{a-1}, p-1,1\right), & b=0\end{cases}
$$

are called basic spin modules. By Lemma 2.18, which holds by [45, Table III] and Lemma 2.17. basic spin modules in characteristic $p$ are exactly the composition factors of the reduction modulo $p$ of basic spin modules in characteristic 0 (indexed by $\left.(n) \in \mathscr{R} \mathscr{P}_{0}(n)\right)$. 
Lemma 2.18. Let $p \geq 3$. Then

- if $p \nmid n$ and $2 \nmid n$ then $S((n), 0) \cong D\left(\beta_{n}, 0\right)$,

- if $p \nmid n$ and $2 \mid n$ then $S((n), \pm) \cong D\left(\beta_{n}, \pm\right)$,

- if $p \mid n$ and $2 \nmid n$ then $S((n), 0) \cong D\left(\beta_{n},+\right) \oplus D\left(\beta_{n},-\right)$,

- if $p \mid n$ and $2 \mid n$ then $S((n), \pm) \cong D\left(\beta_{n}, 0\right)$.

Lemma 2.19 shows that there are cases where it is easy to compute $\mu^{R}$ using the partitions $\beta_{\mu_{i}}$. Here as in the following $\unlhd$ denotes the dominance order.

Lemma 2.19. Let $p \geq 3$ and $\mu=\left(\mu_{1}, \ldots, \mu_{h(\mu)}\right) \in \mathscr{R} \mathscr{P}_{0}(n)$. Then $\mu^{R} \unlhd \sum \beta_{\mu_{i}}$ with equality holding if and only if $\mu_{i} \geq \mu_{i+1}+p$ for $1 \leq i<h(\mu)$. Further if $\mu_{i} \geq \mu_{i+1}+p+\delta_{p \mid \mu_{i+1}}$ and $\nu \in \mathscr{R} \mathscr{P}_{0}(n)$ with $\nu \not \Perp \mu$ then $\left[\bar{S}(\nu): D\left(\mu^{R}\right)\right]=0$.

Proof. Let

$$
\bar{\mu}:=\cup\left\{(j, p(i-1)+k) \mid(j, k) \in \beta_{\mu_{i}}\right\},
$$

so that the first $p$ columns of $\bar{\mu}$ correspond to $\beta_{\mu_{1}}$, the second $p$ columns to $\beta_{\mu_{2}}$ and so on. Note that $\bar{\mu}$ is not necessarily (the Young diagram of) a partition, but $\bar{\mu}$ and $\sum \beta_{\mu_{i}}$ always have the same number of nodes on any row. Further $\mu$ and $\bar{\mu}$ have the same number of nodes on any ladder. It then easily follows from the definition of $\mu^{R}$ that $\mu^{R} \unlhd \sum \beta_{\mu_{i}}$.

Assume next that $\mu_{i}<\mu_{i+1}+p$ for some $1 \leq i<h(\mu)$. Let $(j, k)$ be the good node of $\beta_{\mu_{i+1}}$. Then $(j+1, p(i-1)+k) \notin \bar{\mu}$ and

$$
(\bar{\mu} \backslash(j, p i+k)) \cup(j+1, p(i-1)+k)
$$

has the same number of nodes as $\mu$ on each ladder. It then follows that $\mu^{R} \neq \sum \beta_{\mu_{i}}$ in this case.

Assume now that $\mu_{i} \geq \mu_{i+1}+p$ for $1 \leq i<h(\mu)$. Let $A$ be the set of all $1 \leq r<h(\mu)$ with $p \mid \mu_{r}=\mu_{r+1}+p$. Then

$$
\sum \beta_{\mu_{i}}=\left(\bar{\mu} \backslash\left\{\left(h\left(\beta_{\mu_{r+1}}\right), p r+1\right) \mid r \in A\right\}\right) \cup\left\{\left(h\left(\beta_{\mu_{r+1}}\right), p r\right) \mid r \in A\right\}
$$

(that is $\sum \beta_{\mu_{i}}$ is obtained from $\bar{\mu}$ by moving the last node in the $(r+1)$-th set of $p$ columns one node to the left for all $r \in A$ ). So $\sum \beta_{\mu_{i}}$ and $\bar{\mu}$ have the same number of nodes on any ladder. Further by assumption that $\mu_{i} \geq \mu_{i+1}+p$ it can be checked that $\sum \beta_{\mu_{i}} \in \mathscr{R} \mathscr{P}_{p}(n)$ and so $\mu^{R}=\sum \beta_{\mu_{i}}$.

Last assume that $\mu_{i} \geq \mu_{i+1}+p+\delta_{p \mid \mu_{i+1}}$. Note that in this case $\bar{\mu}=\sum \beta_{\mu_{i}}=\mu^{R}$ by the last paragraph. Let $\nu \in \mathscr{R} \mathscr{P}_{0}(n)$ with $\nu \unlhd \mu$. By Lemma 2.17 and the above it is enough to prove that $\sum \beta_{\mu_{i}} \not \sum \sum \beta_{\nu_{i}}$. Pick $r$ with $\nu_{1}+\ldots+\nu_{r}>\mu_{1}+\ldots+\mu_{r}$ and define $\bar{\nu}$ similarly to $\bar{\mu}$. Then the first rp columns of $\bar{\nu}$ contain more nodes than the first $r p$ columns of $\bar{\mu}$. In particular the first $r p$ columns of $\sum \beta_{\nu_{i}}$ contain more nodes than the first $r p$ columns of $\sum \beta_{\mu_{i}}$ and so $\left(\sum \beta_{\mu_{i}}\right)^{\prime} \unrhd\left(\sum \beta_{\nu_{i}}\right)^{\prime}$, that is $\sum \beta_{\mu_{i}} \not \Perp \beta_{\nu_{i}}$.

2.5. Module structure. Often we will need to consider the structure of certain modules. We write

$$
W \sim V_{1}|\ldots| V_{h}
$$

if $W$ has a filtration with subquotients $V_{j}$ counted from the bottom and

$$
W \sim\left(V_{1,1}|\ldots| V_{1, h_{1}}\right) \oplus \ldots \oplus\left(V_{k, 1}|\ldots| V_{k, h_{k}}\right)
$$

if $W \cong W_{1} \oplus \ldots \oplus W_{k}$ with $W_{j} \sim V_{j, 1}|\ldots| V_{j, h_{j}}$. 
If $V_{1}, \ldots, V_{h}$ are simple we will also write

$$
W \cong V_{1}|\ldots| V_{h}
$$

if $W$ is uniserial with composition factors $V_{j}$ counted from the bottom and

$$
W \cong\left(V_{1,1}|\ldots| V_{1, h_{1}}\right) \oplus \ldots \oplus\left(V_{k, 1}|\ldots| V_{k, h_{k}}\right)
$$

if $W \cong W_{1} \oplus \ldots \oplus W_{k}$ with $W_{j} \cong V_{j, 1}|\ldots| V_{j, h_{j}}$.

Further for groups $G, H$ and modules $A$ of $F G$ and $B$ of $F H$ we will write $A \otimes B$ for the corresponding modules of $F(G \times H)$.

2.6. Permutation modules. In this subsection we will consider the structure of certain permutation modules and prove some results connecting such permutations modules and the endomorphism ring $\operatorname{End}_{F}(V)$, for $V$ a $\widetilde{\mathrm{S}}_{n}$ or $\widetilde{\mathrm{A}}_{n}$ module.

For $\alpha \in \mathscr{P}(n)$ a partition of $n$ let $S^{\lambda}$ be the reduction modulo $p$ of the Specht module indexed by $\alpha$ (which can be viewed as an $\widetilde{\mathrm{S}}_{n}$-module). Further let $\mathrm{S}_{\alpha}$ be the Young subgroup $\mathrm{S}_{\alpha_{1}} \times \mathrm{S}_{\alpha_{2}} \times \cdots \leq \mathrm{S}_{n}$ and define $M^{\alpha}:=\mathbf{1} \uparrow \widetilde{\mathrm{S}}_{n} \widetilde{\mathrm{S}}_{\alpha}$ to be the corresponding permutation module. It is well known (see for example [22]) that $S^{\alpha} \subseteq M^{\alpha}$. Let $\mathrm{A}_{\alpha}=\mathrm{S}_{\alpha} \cap \mathrm{A}_{n}$. If $\alpha \neq\left(1^{n}\right)$ then $\mathrm{S}_{\alpha}$ contains an odd element, so $\mathrm{S}_{n}$ is a single $\left(\mathrm{A}_{n}, \mathrm{~S}_{\alpha}\right)$ double coset. Hence Mackey's theorem gives that $M^{\alpha} \downarrow \widetilde{\mathrm{A}}_{n} \cong \mathbf{1} \uparrow \widetilde{\mathrm{A}}_{\widetilde{A}_{\alpha}}$.

Lemma 2.20 holds by Frobenius reciprocity and the definition of $M^{\alpha}$.

Lemma 2.20. For any $F \widetilde{\mathrm{S}}_{n}$-module $V$ and any $\alpha \in \mathscr{P}(n)$ we have that

$$
\operatorname{dim} \operatorname{Hom}_{\widetilde{\mathrm{S}}_{n}}\left(M^{\alpha}, \operatorname{End}_{F}(V)\right)=\operatorname{dim} \operatorname{End}_{\widetilde{\mathrm{S}}_{\alpha}}\left(V \downarrow_{\widetilde{\mathrm{S}}_{\alpha}}\right) .
$$

Similarly for any $F \widetilde{\mathrm{A}}_{n}$-module $W$ and any $\left(1^{n}\right) \neq \alpha \in \mathscr{P}(n)$ we have that

$$
\operatorname{dim} \operatorname{Hom}_{\widetilde{\mathrm{A}}_{n}}\left(M^{\alpha}, \operatorname{End}_{F}(W)\right)=\operatorname{dim} \operatorname{End}_{\widetilde{\mathrm{A}}_{\alpha}}\left(W \downarrow_{\widetilde{\mathrm{A}}_{\alpha}}\right) .
$$

We will also use Young modules $Y^{\alpha}$ which can be defined using the following well-known facts contained for example in [23] and [35, §4.6]:

Lemma 2.21. There exist indecomposable $F \mathrm{~S}_{n}$-modules $Y^{\alpha}$ for $\alpha \in \mathscr{P}(n)$ such that $M^{\alpha} \cong Y^{\alpha} \oplus \bigoplus_{\beta \triangleright \alpha}\left(Y^{\beta}\right)^{\oplus m_{\beta, \alpha}}$ for some $m_{\beta, \alpha} \geq 0$. Moreover, $Y^{\alpha}$ can be characterized as the unique indecomposable direct summand of $M^{\alpha}$ such that $S^{\alpha} \subseteq$ $Y^{\alpha}$. Finally, we have $\left(Y^{\alpha}\right)^{*} \cong Y^{\alpha}$ for all $\alpha \in \mathscr{P}(n)$.

In order to prove that in most cases $V \otimes W$ is not irreducible, we will usually prove that $\operatorname{Hom}_{G}\left(\operatorname{End}_{F}(V), \operatorname{End}_{F}(W)\right)$ is not 1-dimensional by studying the modules $\operatorname{End}_{F}(V)$ and $\operatorname{End}_{F}(W)$ separately. This will in many cases be done with Lemma 2.22, which is an analogue of [37, Lemma 4.2] for covering groups of symmetric and alternating groups.

Lemma 2.22. Let $G \in\left\{\widetilde{\mathrm{S}}_{n}, \widetilde{\mathrm{A}}_{n}\right\}$ and $B$ and $C$ be FG-modules. For $\alpha \in \mathscr{P}(n)$ let $b_{\alpha}$ and $c_{\alpha}$ be such that there exist $\varphi_{1}^{\alpha}, \ldots, \varphi_{b_{\alpha}}^{\alpha} \in \operatorname{Hom}_{G}\left(M^{\alpha}, B^{*}\right)$ with $\left.\varphi_{1}^{\alpha}\right|_{S^{\alpha}}, \ldots,\left.\varphi_{b_{\alpha}}^{\alpha}\right|_{S^{\alpha}}$ linearly independent and that similarly there exist $\psi_{1}^{\alpha}, \ldots, \psi_{c_{\alpha}}^{\alpha} \in \operatorname{Hom}_{G}\left(M^{\alpha}, C\right)$ with $\left.\psi_{1}^{\alpha}\right|_{S^{\alpha}}, \ldots,\left.\psi_{c_{\alpha}}^{\alpha}\right|_{S^{\alpha}}$ linearly independent. Then

$$
\operatorname{dim} \operatorname{Hom}_{G}(B, C) \geq \sum_{\alpha \in D} b_{\alpha} c_{\alpha},
$$

where $D=\mathscr{P}_{p}(n)$ if $G=\widetilde{\mathrm{S}}_{n}$ or $D=\left\{\alpha \in \mathscr{P}_{p}(n) \mid \alpha>\alpha^{\mathrm{M}}\right\}$ if $G=\widetilde{\mathrm{A}}_{n}$. 
Since we will often consider permutation modules $M^{(n-m, \mu)}$ for certain fixed partitions $\mu \in \mathscr{P}(m)$ (with $m$ small), we will write $M_{\mu_{1}, \mu_{2}, \ldots}$ (or $M_{\mu}$ ) for the module $M^{(n-m, \mu)}$. Similarly we will write $D_{\mu}, S_{\mu}$ and $Y_{\mu}$ for $D^{(n-m, \mu)}, S^{(n-m, \mu)}$ and $Y^{(n-m, \mu)}$ (when they are defined).

2.7. Hooks. We now consider the structure of the reduction modulo $p$ of Specht modules indexed by hook partitions. Such modules have a quite easy structure, since $p \neq 2$.

For $0 \leq k \leq n-1-\delta_{p \mid n}$ define

$$
\bar{D}_{n, k}=\bar{D}_{k}:= \begin{cases}D^{\left(n-k,(k)^{\mathrm{M}}\right)}, & k<n(p-1) / p, \\ D^{\left((k+1)^{\mathrm{M}}, n-k-1\right)}, & k \geq n(p-1) / p \text { and } p \nmid n, \\ D^{\left((k+2)^{\mathrm{M}}, n-k-2\right)}, & k \geq n(p-1) / p \text { and } p \mid n .\end{cases}
$$

Note that for $k<p$ we then have that $\bar{D}_{k}=D_{1^{k}}$ (unless $\left.k=p-1=n-1\right)$. Define $\mathscr{H}_{p}(n):=\left\{\left(a,(b)^{\mathrm{M}}\right),\left((c)^{\mathrm{M}}, d\right)\right\} \cap \mathscr{P}_{p}(n)$, so that $\mathscr{H}_{p}(n)$ is the set of partition labeling the modules $\bar{D}_{k}$.

Lemma 2.23 holds by [21, p. 52] and [42, Theorem 2].

Lemma 2.23. Let $p \geq 3$. Then for $0 \leq k \leq n-1$ :

- if $p \nmid n$ then $S_{1^{k}} \cong \bar{D}_{k}$,

- if $p \mid n$ then $S_{1^{k}} \cong \bar{D}_{k-1} \mid \bar{D}_{k}$, where $\bar{D}_{-1}=\bar{D}_{n-1}=0$.

The following properties then easily follow:

Lemma 2.24. Let $c=1$ if $p \nmid n$ or $c=2$ if $p \mid n$. Then $\bar{D}_{k} \cong \bar{D}_{n-c-k} \otimes \operatorname{sgn}$ for each $0 \leq k \leq n-c$. In particular $\bar{D}_{k} \cong \bar{D}_{k} \otimes \operatorname{sgn}$ if and only if $k=(n-c) / 2$.

Lemma 2.25. Let $p \geq 3$. Then $\lambda \in \mathscr{H}_{p}(n)$ if and only if $\lambda^{\mathrm{M}} \in \mathscr{H}_{p}(n)$.

If $k \neq\left(n-1-\delta_{p \mid n}\right) / 2$ we will then write $\bar{E}_{k}$ for $\bar{D}_{k \downarrow_{\mathrm{A}_{n}}}$. On the other hand if $k=\left(n-1-\delta_{p \mid n}\right) / 2$ we will then write $\bar{E}_{k, \pm}$ for the composition factors of $\bar{D}_{k \downarrow} \downarrow_{\mathrm{A}_{n}}$. When working for $\widetilde{\mathrm{S}}_{n}$ and $\widetilde{\mathrm{A}}_{n}$ at the same time, we will often write $\bar{D}_{k}$ to also indicate its restriction to $\widetilde{\mathrm{A}}_{n}$.

\section{SPECIAL HOMOMORPHISMS}

In this section, for $G=\widetilde{\mathrm{S}}_{n}$ or $\widetilde{\mathrm{A}}_{n}$, we will prove that for certain large classes of modules $V$ there exist homomorphisms $\psi \in \operatorname{Hom}_{G}\left(M, \operatorname{End}_{F}(V)\right)$ with $M=M_{\mu}$ or $S_{\mu}$ which do not vanish on $S_{\mu}$.

3.1. Definition of homomorphisms. We now define certain special elements $x_{\mu}$. Using these elements we will then define the homomorphisms that will play a role in this section. After having proved some branching rules in 33.2 , we will then prove in $\$ 3.3$ that these homomorphisms do not vanish on $S^{\mu}$ for large classes of modules $V$. For $k \geq 3$ odd let $C_{k}^{+}$and $C_{k}^{-}$be the conjugacy classes in $\widetilde{\mathrm{A}}_{k+1}$ of $(1,2,3, \ldots, k)$ and $(2, \widetilde{1,3, \ldots, k})$ respectively (so that $C_{k}^{ \pm}$are the two conjugacy classes in $\widetilde{\mathrm{A}}_{k+1}$ consisting of the odd order lifts of $k$-cycles). Note that since $k \geq 3$ is odd the conjugacy classes $C_{k}^{+}$and $C_{k}^{-}$are distinct, as $(k, 1)$ has odd distinct parts and so $(2,1,3, \ldots, k)=(1,2)(1,2,3, \ldots, k)(1,2)$ is not in the $A_{k+1}$ conjugacy class of $(1,2,3, \ldots, k)$. 
Define

$$
\begin{aligned}
x_{3} & \left.=\sum_{g \in \mathrm{S}_{\{1,4\}} \times \mathrm{S}_{\{2,5\}} \times \mathrm{S}_{\{3,6\}}} \operatorname{sgn}(g) \widetilde{g}(\widetilde{(1,2,3})+(\widetilde{1,3,2})\right)(\widetilde{g})^{-1}, \\
x_{3,1^{2}} & :=\sum_{g \in \mathrm{S}_{4,2,2}} \sum_{h \in \mathrm{S}_{\{2,6,8\}}} \operatorname{sgn}(g) \widetilde{g} \widetilde{h}(2, \widetilde{6,8,3}, 4)(\widetilde{h})^{-1}(\widetilde{g})^{-1}, \\
x_{1^{k}} & :=\sum_{g \in C_{k}^{+}} \widetilde{g}-\sum_{g \in C_{k}^{-}} \widetilde{g},
\end{aligned}
$$

where $x_{1^{k}}$ is defined only for $k \geq 3$ odd.

Let $h \in \mathrm{S}_{n}$ have odd order. By definition $\widetilde{h}$ also has odd order. So, for any $g \in \mathrm{S}_{n}, \widetilde{g} \widetilde{h} \widetilde{g}^{-1}=\widetilde{g h g^{-1}}$ is the lift of odd order of $g h g^{-1}$ and this element does not depend on the choice of lift of $g$. This fact will be used in the proofs of Lemmas 3.1 , 3.2 and 3.3 .

In the next statements we will use standard bases for the modules $M^{\mu}$ and $S^{\mu}$. For definitions of such bases see [22, $\S 4,8.4]$. When considering a basis element corresponding to a certain tableau, we will just write elements appearing below the first row of the tableau.

Lemma 3.1. Let $n \geq 6, G \in\left\{\widetilde{\mathrm{S}}_{n}, \widetilde{\mathrm{A}}_{n}\right\}$ and $V$ be an $F G$-module. If $x_{3} V \neq 0$ then there exists $\psi \in \operatorname{Hom}_{G}\left(M_{3}, \operatorname{End}_{F}(V)\right)$ which does not vanish on $S_{3}$.

Proof. If $V$ is also a representation of $\mathrm{S}_{n}$ this result is just [31, Lemma 6.1]. For $p>3$ it can be recovered from the proof of [34, Theorem 7.2] and the case $p=3$ could be similarly obtained, but we will prove the result here in the general setting.

Let $\left\{v_{a, b, c} \mid 1 \leq a<b<c \leq n\right\}$ be the standard basis of $M_{3}$ (by identifying a tabloid with its second row). Define $\psi: M_{3} \rightarrow \operatorname{End}_{F}(V)$ through

$$
\left.\psi\left(v_{a, b, c}\right)(w)=(\widetilde{(a, b, c)}+\widetilde{(a, c, b})\right) w .
$$

For any $g \in \mathrm{S}_{n}, 0 \leq x \leq 1$ and any pairwise distinct $1 \leq a, b, c \leq n$ we have by definition of the standard basis of $M_{3}$ that $z^{x} \widetilde{g} v_{a, b, c}=v_{g(a), g(b), g(c)}$ (up to reordering the indexes of $\left.v_{g(a), g(b), g(c)}\right)$ and so, provided $g \in \mathrm{A}_{n}$ if $G=\widetilde{\mathrm{A}}_{n}$,

$$
\begin{aligned}
\psi\left(z^{x} \widetilde{g} v_{a, b, c}\right)(w) & =\psi\left(v_{g(a), g(b), g(c)}\right)(w) \\
& =((g(a) \widetilde{, g(b)}, g(c))+(g(a) \widetilde{g(c)}, g(b))) w \\
& \left.=z^{x} \widetilde{g}(\widetilde{(a, b, c)}+\widetilde{(a, c, b})\right)\left(z^{x} \widetilde{g}\right)^{-1} w \\
& =\left(z^{x} \widetilde{g} \psi\left(v_{a, b, c}\right)\right)(w) .
\end{aligned}
$$

Thus $\psi \in \operatorname{Hom}_{G}\left(M_{3}, \operatorname{End}_{F}(V)\right)$. Let now $t$ be the standard basis vector of $S_{3}$ corresponding to

$$
\begin{array}{llllll}
1 & 2 & 3 & 7 & \cdots & n \\
4 & 5 & 6 & & &
\end{array}
$$

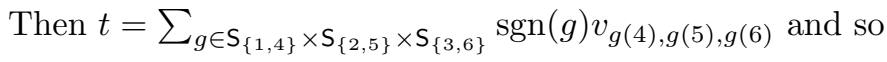

$$
\begin{aligned}
\psi(t)(w) & =\sum_{g \in \mathrm{S}_{\{1,4\}} \times \mathrm{S}_{\{2,5\}} \times \mathrm{S}_{\{3,6\}}} \operatorname{sgn}(g)((g(4) \widetilde{, g(5)}, g(6))+(g(4) \widetilde{, g(6), g}(5))) w \\
& =-x_{3} w .
\end{aligned}
$$

It follows that $\psi$ does not vanish on $S_{3}$ if $x_{3} V \neq 0$. 
Lemma 3.2. Let $n \geq 8, G \in\left\{\widetilde{\mathrm{S}}_{n}, \widetilde{\mathrm{A}}_{n}\right\}$ and $V$ be an $F G$-module. If $x_{3,1^{2}} V \neq 0$ then there exists $\psi \in \operatorname{Hom}_{G}\left(M_{3,1^{2}}, \operatorname{End}_{F}(V)\right)$ which does not vanish on $S_{3,1^{2}}$.

Proof. Let $\left\{v_{\{a, b, c\}, d, e} \mid 1 \leq a, b, c, d, e \leq n\right.$ pairwise distinct $\}$ be the standard basis of $M_{3,1^{2}}$ (here $v_{\{a, b, c\}, d, e}$ corresponds to the tabloid with the elements of $\{1, \ldots, n\} \backslash$ $\{a, b, c, d, e\}$ in the first row, $a, b, c$ in the second row, $d$ in the third row and $e$ in the fourth row). Define $\psi: M_{3,1^{2}} \rightarrow \operatorname{End}_{F}(V)$ through

$$
\psi\left(v_{\{a, b, c\}, d, e}\right)(w)=\sum_{h \in \mathrm{S}_{\{a, b, c\}}} \tilde{h}(a, \widehat{b, c, d}, e) \widetilde{h}^{-1} w
$$

for $w \in V$. Then similarly to the previous lemma $\psi \in \operatorname{Hom}_{G}\left(M_{3,1^{2}}, \operatorname{End}_{F}(V)\right)$ and if $t$ is the element of the standard basis of $S_{3,1^{2}}$ corresponding to

$$
\begin{array}{llllll}
1 & 5 & 7 & 9 & \cdots & n \\
2 & 6 & 8 & & & \\
3 & & & & & \\
4 & & & & &
\end{array}
$$

then $\psi(t)$ is just multiplication with $x_{3,1^{2}}$ (by definitions of $t$ and $\psi$ ).

Lemma 3.3. Let $G \in\left\{\widetilde{\mathrm{S}}_{n}, \widetilde{\mathrm{A}}_{n}\right\}$ and $V$ be an $F G$-module. If $k \geq 3$ is odd, $n>k$ and $x_{1^{k}} V \neq 0$ then there exists $0 \neq \psi \in \operatorname{Hom}_{G}\left(S_{1^{k}}, \operatorname{End}_{F}(V)\right)$. If $p \nmid k$ then $\psi$ extends to $\varphi \in \operatorname{Hom}_{G}\left(M_{1^{k}}, \operatorname{End}_{F}(V)\right)$.

Proof. Let $\left\{v_{b_{1}, \ldots, b_{k}}: 1 \leq b_{j} \leq n\right.$ pairwise distinct $\}$ and $\left\{w_{b_{1}, \ldots, b_{k}}: 2 \leq b_{1}<\right.$ $\left.\ldots<b_{k} \leq n\right\}$ be the standard bases of $M_{1^{k}}$ and $S_{1^{k}}$ respectively. Since $k \geq$ 3 is odd, the cycles $\left(b_{1}, b_{2}, b_{3}, \ldots, b_{k}\right)$ and $\left(b_{2}, b_{1}, b_{3}, \ldots, b_{k}\right)$ are not conjugate in $\mathrm{A}_{\left\{1, b_{1}, \ldots, b_{k}\right\}}$. It follows that also $\left(b_{1}, \widetilde{\left.b_{2}, b_{3}, \ldots, b_{k}\right)}\right.$ and $\left(b_{2}, \widetilde{b_{1}, b_{3}, \ldots, b_{k}}\right)$ are not conjugated in $\widetilde{\mathrm{A}}_{\left\{1, b_{1}, \ldots, b_{k}\right\}}$ (and so their $\widetilde{\mathrm{A}}_{\left\{1, b_{1}, \ldots, b_{k}\right\} \text {-conjugacy classes are the two }}$ distinct conjugacy classes of odd ordered lifts of $k$-cycles). For $w \in V$ define

$$
\begin{aligned}
\bar{\varphi}\left(v_{b_{1}, \ldots, b_{k}}\right)(w) & :=\left(\widetilde{b_{1}, \ldots, b_{k}}\right) w, \\
\psi\left(w_{b_{1}, \ldots, b_{k}}\right)(w) & :=\sum_{g \in C_{b_{1}, \ldots, b_{k}}^{+}} g w-\sum_{g \in C_{b_{1}, \ldots, b_{k}}^{-}} g w,
\end{aligned}
$$

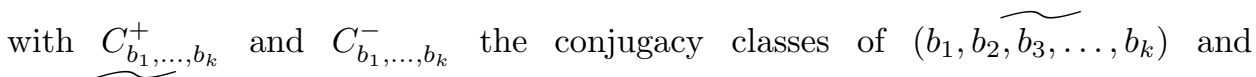
$\left(b_{2}, b_{1}, b_{3}, \ldots, b_{k}\right)$ in $\widetilde{\mathrm{A}}_{\left\{1, b_{1}, \ldots, b_{k}\right\}}$.

Then $\bar{\varphi} \in \operatorname{Hom}_{G}\left(M_{1^{k}}, \operatorname{End}_{F}(V)\right)$ and $\psi \in \operatorname{Hom}_{G}\left(S_{1^{k}}, \operatorname{End}_{F}(V)\right)$ (similarly to Lemma 3.1). Since $\psi\left(w_{2, \ldots, k+1}\right)$ is given by multiplication with $\pm x_{1^{k}}$, the first part of the lemma follows. The second part follows from

$$
\left.\bar{\varphi}\right|_{S_{1 k}}=\left|C_{\mathrm{S}_{k+1}}\left(b_{1}, b_{2}, b_{3}, \ldots, b_{k}\right)\right| \psi=k \psi .
$$

3.2. Branching recognition. In order to check that in most cases if $V$ is an irreducible representation of $\widetilde{\mathrm{S}}_{n}$ or $\widetilde{\mathrm{A}}_{n}$ we have that $x_{\mu} V \neq 0$ (for $x_{\mu}$ one of the elements defined in the previous section); we will prove that $x_{\mu} W \neq 0$, for $W$ a composition factor of $V \downarrow_{\widetilde{S}_{m}}$ or $V \downarrow_{\widetilde{A}_{m}}$ with $m$ small (depending on $\mu$ ). In order to do this, we will prove in this section that the restrictions $V \downarrow_{\widetilde{S}_{m}}$ and $V \downarrow_{\widetilde{A}_{m}}$ often contain modules indexed by partitions with similar property as the partition indexing $V$. 
Lemma 3.4 ([34, Lemma 2.4]). Let $p \geq 3, n \geq 6$ and $\lambda \in \mathscr{R} \mathscr{P}_{p}(n) \backslash\left\{\beta_{n}\right\}$. Then there exists $\mu \in \mathscr{R} \mathscr{P}_{p}(n-1) \backslash\left\{\beta_{n-1}\right\}$ such that $D(\mu)$ is a composition factor of $D(\lambda) \downarrow \widetilde{\mathrm{s}}_{n-1}$.

Lemma 3.5. Let $p=3, n \geq 9$ and $\lambda=\left(\lambda_{1}, \lambda_{2}\right)$ with $\lambda_{1} \geq \lambda_{2}+2 \geq 5$. Then there exists $\mu=\left(\mu_{1}, \mu_{2}\right)$ with $\mu_{1} \geq \mu_{2}+2 \geq 5$ such that $D^{\mu}$ is a composition factor of $D^{\lambda} \downarrow_{S_{n-1}}$.

Proof. If $\lambda_{1} \geq \lambda_{2}+3$ then $D^{\left(\lambda_{1}-1, \lambda_{2}\right)}$ is a composition factor of $D^{\lambda} \downarrow_{S_{n-1}}$ by Lemma 2.2. If $\lambda_{1}=\lambda_{2}+2$ then $\lambda_{2} \geq 3$ and $D^{\left(\lambda_{1}, \lambda_{2}-1\right)}$ is a composition factor of $D^{\lambda} \downarrow_{S_{n-1}}$ by the same lemma.

Lemma 3.6. Let $p \geq 3, n \geq 7$ and $\lambda \in \mathscr{P}_{p}(n) \backslash \mathscr{H}_{p}(n)$. Then there exists a composition factor of $D^{\lambda} \downarrow_{S_{n-1}}$ of the form $D^{\mu}$ with $\mu \in \mathscr{P}_{p}(n-1) \backslash \mathscr{H}_{p}(n-1)$.

Assume now that $n \geq 10$. If further $h(\lambda), h\left(\lambda^{\mathrm{M}}\right) \geq 3$, then there exists $\mu \in$ $\mathscr{P}_{p}(n-1) \backslash \mathscr{H}_{p}(n-1)$ with $h(\mu), h\left(\mu^{\mathrm{M}}\right) \geq 3$ and such that $D^{\mu}$ a composition factor of $D^{\lambda} \downarrow_{S_{n-1}}$.

Proof. Throughout the proof we will use Lemma 2.2 without further reference to it. By Lemma 2.25 we have that $\mathscr{H}_{p}(n)$ is fixed under the Mullineux map. So the lemma holds for $\lambda$ if and only if it holds for $\lambda^{\mathrm{M}}$.

We may assume (up to taking $\lambda^{\mathrm{M}}$ ) that $\lambda$ has a good node $A$ such that $\mu=$ $\lambda \backslash A \in \mathscr{H}_{p}(n-1)$ or that $n \geq 10, \lambda=\left(\lambda_{1}, \lambda_{2}, 1\right), h\left(\lambda^{\mathrm{M}}\right) \geq 3,(3,1)$ is good, while $\left(1, \lambda_{1}\right)$ and $\left(2, \lambda_{2}\right)$ are not good.

Case 1. $n \geq 10, \lambda=\left(\lambda_{1}, \lambda_{2}, 1\right), h\left(\lambda^{\mathrm{M}}\right) \geq 3,(3,1)$ is good, while $\left(1, \lambda_{1}\right)$ and $\left(2, \lambda_{2}\right)$ are not good.

Case 1.1. $p=3$. In this case we may assume that $\lambda_{1} \geq \lambda_{2}+2 \geq 5$, since else $\lambda \in \mathscr{H}_{p}(n)$. If $\lambda_{1} \geq \lambda_{2}+3$ then let $B:=\left(1, \lambda_{1}\right)$. If $\lambda_{1}=\lambda_{2}+2$ then $\lambda_{2} \geq 4$. In this case let $B:=\left(2, \lambda_{2}\right)$. Then $B$ is normal in $\lambda, \lambda \backslash B \notin \mathscr{H}_{p}(n-1)$ and it can be easily checked that $h(\lambda \backslash B)=3$ and $h\left((\lambda \backslash B)^{\mathrm{M}}\right)=5$.

Case 1.2. $p \geq 5$. In this case we may assume that $\lambda_{2} \geq 2$. Further $\lambda_{1} \geq 5$. If $\lambda_{1}>\lambda_{2}$ let $B:=\left(1, \lambda_{1}\right)$. If $\lambda_{1}=\lambda_{2}$ let $B:=\left(2, \lambda_{2}\right)$. Then $B$ is normal, $\lambda \backslash B \notin \mathscr{H}_{p}(n)$ and $h(\lambda \backslash B)=3$. Since $(\lambda \backslash B)_{1} \geq 4$, the $p$-rim of $\lambda \backslash B$ contains at least $\min \left\{p+\delta_{p=5},(\lambda \backslash B)_{1}+2\right\} \geq 6$ nodes (where $\delta_{p=5}=1$ if $p=5$ and 0 else). So $h\left((\lambda \backslash B)^{\mathrm{M}}\right) \geq 3$.

Case 2. $\mu=(n-1)$. Then $\lambda \in\{(n),(n-1,1)\}$, so $\lambda \in \mathscr{H}_{p}(n)$, contradicting the assumptions.

Case 3. $\mu=(n-1)^{\mathrm{M}}$. In this case $(n)$ can be obtained from $\lambda^{\mathrm{M}}$ by removing a good node by Lemma 2.5. So this case follows from Case 2

Case 4. $\mu=\left(n-k-1,1^{k}\right)$ with $1 \leq k \leq p-2$. Then $\lambda \in\left\{\left(n-k, 1^{k}\right),(n-k-\right.$ $\left.\left.1,1^{k+1}\right),\left(n-k-1,2,1^{k-1}\right)\right\}$, so we may assume that $\lambda=\left(n-k-1,2,1^{k-1}\right)$. Let $B:=(1, n-k-1)$.

Case 4.1. $n-k \geq p+3$ or $n-k=p+1$. In this case $B$ is normal in $\lambda$ and $\lambda \backslash B \notin \mathscr{H}_{p}(n-1)$. Further the first columns of the Mullineux symbols of $\lambda$ and $\lambda \backslash B$ are equal. Thus $h(\lambda)=h(\lambda \backslash B)$ and $h\left(\lambda^{\mathrm{M}}\right)=h\left((\lambda \backslash B)^{\mathrm{M}}\right)$ and then the lemma holds. 
Case 4.2. $n-k=p+2$. In this case $n \leq 2 p$ and so $p \geq 5$. Again $B$ is normal and $\lambda \backslash B \notin \mathscr{H}_{p}(n-1)$. Further $h(\lambda \backslash B)=h(\lambda)$ and, since the first column of the Mullineux symbol of $\lambda \backslash B$ is $\left(\begin{array}{c}p+k-1 \\ k+1\end{array}\right)$, we have that $h\left((\lambda \backslash B)^{\mathrm{M}}\right) \geq p-2 \geq 3$. So the lemma holds.

Case 4.3. $4 \leq n-k \leq p$. We may assume that $(n-k, k) \neq(4, p-3)$, since else $\lambda \backslash B=(n-1)^{\mathrm{M}}$, which was already covered in Case 3 (since $B$ is normal). In this case $n \leq 2 p-2$ and so $p \geq 5$. Then $B$ is normal and $\lambda \backslash B \notin \mathscr{H}_{p}(n-1)$. Further $h(\lambda \backslash B)=h(\lambda)$ and $h\left((\lambda \backslash B)^{\mathrm{M}}\right) \geq n-k-1 \geq 3$. So the lemma holds.

Case 4.4. $n-k=3$. In this case $\lambda=\left(2^{2}, 1^{k-1}\right)$. If $k=p-2$ then $\lambda \in \mathscr{H}_{p}(n)$, so, since $n \geq 7$, we may assume that $4 \leq k \leq p-3$ (so in particular $p \geq 7$ ). In this case $\lambda^{\mathrm{M}}=(k+1,2)$, so we only have to prove the first part of the lemma, which follows from $C:=(1, k+1)$ being normal in $\lambda^{\mathrm{M}}$ and from $\lambda^{\mathrm{M}} \backslash C \notin \mathscr{H}_{p}(n-1)$.

Case 5. $h(\mu)=p$. By Lemmas 2.5 and 2.24 we may assume that $\mu=\left(c,(d)^{\mathrm{M}}\right)$ with $c+d=n-1$ and $c>d \geq p-1$ (otherwise $\mu^{\mathrm{M}}$ is of this form or of one of the forms considered in Cases 2 4). In this case

$$
\lambda \in\left\{\left(c+1,(d)^{\mathrm{M}}\right),\left(c,(d+1)^{\mathrm{M}}\right),\left(c,(d)^{\mathrm{M}}, 1\right),\left(c,(d)^{\mathrm{M}}\right) \cup\left(2,(d)_{1}^{\mathrm{M}}+1\right)\right\} .
$$

We may assume that $d \geq p$ and $\lambda=\left(c,(d)^{\mathrm{M}}, 1\right)$ or that $(p-1) \nmid d$ and $\lambda=\left(c,(d)^{\mathrm{M}}\right) \cup$ $\left(2,(d)_{1}^{\mathrm{M}}+1\right)$. From $c>d \geq p$ and since $c+d=n-1$ we have $p \leq d \leq(n-2) / 2$ and so

$$
\begin{aligned}
\lambda_{1}-\lambda_{2} & \geq c-\left(\left\lceil\frac{d}{p-1}\right\rceil+1\right) \\
& =(n-d-1)-\left(\left\lceil\frac{d}{p-1}\right\rceil+1\right) \\
& \geq n-2-\left\lceil\frac{p d}{p-1}\right\rceil \\
& \geq\left\lfloor(n-2) \frac{p-2}{2(p-1)}\right\rfloor \\
& \geq\left\lfloor\frac{p(p-2)}{p-1}\right\rfloor \\
& =\left\lfloor\frac{(p-1)^{2}-1}{p-1}\right\rfloor \\
& =p-2 .
\end{aligned}
$$

Case 5.1. $p \geq 5$. Let $B:=(1, c)$. Then $B$ is normal, $\lambda \backslash B \notin \mathscr{H}_{p}(n-1)$ and $h(\lambda \backslash B)=h(\lambda)$. Further the $p$-rim of $\lambda \backslash B$ contains at least

$$
\min \left\{(\lambda \backslash B)_{1}-(\lambda \backslash B)_{2}+1, p\right\}+h(\lambda \backslash B)-1 \geq h(\lambda \backslash B)+p-2
$$

nodes. So the first column of the Mullineux symbol of $\lambda \backslash B$ is $\left(\begin{array}{c}\geq h(\lambda \backslash B)+p-2 \\ h(\lambda \backslash B)\end{array}\right)$ and then $h\left((\lambda \backslash B)^{\mathrm{M}}\right) \geq p-2 \geq 3$.

Case 5.2. $p=3$. Again let $B:=(1, c)$. If $h(\lambda)=3$ then the $p$-rim contains at least $\min \left\{(\lambda \backslash B)_{1}-(\lambda \backslash B)_{2}+1, p\right\}+2$ nodes. If $h(\lambda)=4$ then the $p$-rim contains at least $\min \left\{(\lambda \backslash B)_{1}-(\lambda \backslash B)_{2}+1, p\right\}+3$ nodes. If $\lambda_{1}-\lambda_{2}=(\lambda \backslash B)_{1}-(\lambda \backslash B)_{2}+1 \geq 3$ we can then argue in either case as in Case 5.1. So assume that $\lambda_{1}-\lambda_{2} \leq 2$. Then $c-(d+1) / 2-1 \leq \lambda_{1}-\lambda_{2} \leq 2$. Since $c \geq n / 2$ and $d \leq(n-2) / 2$ it follows that $n / 2 \leq c \leq n / 4+3$. So $n \leq 12$ and it can then be easily checked 
that $\lambda \in\left\{\left(4,2,1^{2}\right),(5,3,1),(6,4,2)\right\}$. In the first case $D^{\left(3,2,1^{2}\right)}$ gives a composition factor of $D^{\lambda} \downarrow_{S_{n-1}}$ as wanted, in the second case $D^{(5,3)}$, in the third case $D^{(6,4,1)}$.

3.3. Endomorphisms rings. We are now ready to study the endomorphisms rings $\operatorname{End}_{F}(V)$ for $V$ simple $F \widetilde{\mathrm{S}}_{n^{-}}$or $F \widetilde{\mathrm{A}}_{n}$-modules indexed by certain (large) families of partitions. We will use the elements $x_{\mu}$ defined at the beginning of $\$ 3.1$.

In the proofs of this subsection, results considering the number of elements in conjugacy classes of $\mathrm{S}_{m}$ or $\widetilde{\mathrm{S}}_{m}$ have been obtained with GAP 18 . Character tables for spin irreducible characters in characteristic 0 for $n \leq 13$ can be found in [40. These character tables allow us to compute spin character values in this subsection, after having computed if the lifts of elements of odd order on which characters of basic spin modules in characteristic 0 take positive value have odd or even order using [40, pp. 57-58] (the representation $\Gamma_{n}^{*}$ defined there is isomorphic is the representation we denoted by $S((n))$; see [44, $\S 3])$.

Lemma 3.7. Let $p \geq 3, n \geq 6$ and $\lambda \in \mathscr{P}_{p}(n)$. If $h(\lambda), h\left(\lambda^{\mathrm{M}}\right) \geq 3$ and $V$ is a simple $F_{\mathrm{S}^{-}}$or $F \mathrm{~A}_{n}$-module indexed by $\lambda$ then there exists $\varphi: M_{3} \rightarrow \operatorname{End}_{F}(V)$ which does not vanish on $S_{3}$.

Proof. In view of Lemma 3.1 if $x_{3} V \neq 0$ the result holds. If $V \cong D^{\lambda}$ (and then also if $V \cong E^{\lambda}$ ) we have that $x_{3} V \neq 0$ by [11, Proposition 3.8] if $p \geq 5$ or by [31, Lemma $6.6]$ if $p=3$. So we may assume that $V \cong E_{ \pm}^{\lambda}$. Since $D^{\lambda} \downarrow_{\mathrm{A}_{n}} \cong E_{+}^{\lambda} \oplus E_{-}^{\lambda}$ there exists $\varepsilon \in\{ \pm\}$ such that $x_{3} E_{\varepsilon}^{\lambda} \neq 0$ and then the result holds for $E_{\varepsilon}^{\lambda}$. Since $E_{+}^{\lambda} \cong\left(E_{-}^{\lambda}\right)^{\sigma}$ for $\sigma \in \mathrm{S}_{n} \backslash \mathrm{A}_{n}$, the result holds also for $E_{-\varepsilon}^{\lambda}$.

Lemma 3.8. Let $p \geq 3, n \geq 6$ and $\lambda \in \mathscr{R} \mathscr{P}_{p}(n) \backslash\left\{\beta_{n}\right\}$. If $V$ is a simple $F \widetilde{\mathrm{S}}_{n^{-}}$ or $F \widetilde{\mathrm{A}}_{n}$-module indexed by $\lambda$ then there exists $\varphi: M_{3} \rightarrow \operatorname{End}_{F}(V)$ which does not vanish on $S_{3}$.

Proof. For $p \geq 5$ this holds by [34, Theorem 7.2] (and its proof). So we may assume that $p=3$.

From Lemma 3.1 it is enough to prove that $x_{3} V \neq 0$. From Lemma 3.4, since $\lambda \neq \beta_{n}$, there exists a composition factor of $V \downarrow_{\widetilde{A}_{6}}$ of the form $E((4,2), \pm)$. So it is enough to prove that $x_{3} E((4,2), \pm) \neq 0$. Let $W((6), 0)$ be the reduction modulo 3 of the basic spin module of $\widetilde{\mathrm{A}}_{6}$ in characteristic 0 and $W((4,2), \pm)$ be the reduction modulo 3 of the simple spin modules of $\widetilde{\mathrm{A}}_{6}$ indexed by $(4,2)$ in characteristic 0 . Let $\chi^{(6), 0}$ and $\chi^{(4,2), \pm}$ be the characters of the reduction modulo 3 of $W((6), 0)$ and $W((4,2), \pm)$ respectively. Using decomposition matrices and Lemma 2.17 it can be checked that the characters of $E((4,2), \pm$ ) (over the field $F$ ) are $\chi^{ \pm}=\chi^{(4,2), \pm}-\chi^{(6), 0}$. In order to prove that $x_{3} E((4,2), \pm) \neq 0$ it is enough to prove that $\chi^{ \pm}\left(x_{3} y\right) \neq 0$ for some $y \in \tilde{\mathrm{A}}_{6}$. Let $y:=(1,5, \widetilde{5,3)}(4,6)$. It can be computed that, up to exchange of $y$ with $z y, x_{3} y$ is given by

$$
\begin{aligned}
& z^{\cdots}\left(1,5 \widetilde{, 3,2)}(4,6)+z^{\cdots}\left(1, \widetilde{1,5)(4,6)}+z^{\cdots}(1, \widehat{3)} \widetilde{(2,4}, 6,5)+z^{\cdots}(1,4, \widetilde{6,3)}(2,5)\right.\right. \\
& +(1, \widetilde{5,6,2}, 3)+z(1, \widetilde{5,4,2}, 3)+(1, \widetilde{6, \widetilde{4})(2,3}, 5)+(2, \widetilde{3,6,4}, 5) \\
& -z(1, \widehat{5,3)(2,4}, 6)-z(1, \widehat{5,4,6}, 3)-z^{\cdots}\left(1,3, \widetilde{5,2)}(4,6)-z^{\cdots}(\widetilde{2,5)(4,6)}\right. \\
& -z^{\cdots}\left(1,5 \widetilde{6,4)}(2,3)-z^{\cdots}(1,5 \widetilde{(2,3,6}, 4)-z(\widetilde{1,6,5,2}, 3)-(1, \widetilde{4,5,2}, 3)\right. \text {. }
\end{aligned}
$$


Let $C^{+}$and $C^{-}$be the two conjugacy classes of lifts in $\tilde{\mathrm{A}}_{6}$ of elements of $\mathrm{A}_{6}$ with cycle partition $(4,2)$. Up to exchange of $C^{+}$and $C^{-}$, it can be computed that the lifts of $(1,5,3,2)(4,6),(1,3)(2,4,6,5),(1,3,5,2)(4,6)$ and $(1,5)(2,3,6,4)$ appearing in $x_{3} y$ are all in $C^{+}$, while those of $(1,4,6,3)(2,5)$ and $(1,5,6,4)(2,3)$ are in $C^{-}$. Since all lifts of elements of the form $(a, b)(c, d)$ are conjugated in $\widetilde{\mathrm{A}}_{6}$, it then follows from $\chi^{ \pm}=\chi^{(4,2), \pm}-\chi^{(6), 0}$ that

$$
\chi^{ \pm}\left(x_{3} y\right)=2 \chi^{ \pm}((1, \widetilde{2,3,4}, 5))+2 \chi^{ \pm}((1, \widetilde{2,3)(4,5}, 6)) \equiv 2 \bmod 3,
$$

so the lemma holds.

Lemma 3.9. Let $p=3, n \geq 8, G \in\left\{\mathrm{S}_{n}, \mathrm{~A}_{n}\right\}$ and $\lambda=\left(\lambda_{1}, \lambda_{2}\right) \in \mathscr{P}_{3}(n)$ with $\lambda_{1} \geq \lambda_{2}+2 \geq 5$. Let $V$ be an irreducible $F G$-module indexed by $\lambda$. Then there exists $\psi \in \operatorname{Hom}_{G}\left(M_{3,1^{2}}, \operatorname{End}_{F}(V)\right)$ which does not vanish on $S_{3,1^{2}}$.

Proof. By [33. Lemma 1.8], $\lambda \neq \lambda^{\mathrm{M}}$, so $V \cong D^{\lambda}$ or $E^{\lambda}$. So it is enough to prove the lemma for $G=\mathrm{S}_{n}$. From Lemma 3.2 it is enough to prove that $x_{3,1^{2}} D^{\lambda} \neq 0$. Throughout this proof we will consider $x_{3,1^{2}}$ as an element of $F \mathrm{~S}_{8}$ instead of $F \widetilde{\mathrm{S}}_{8}$ by sending $\widetilde{g}$ to $g$. Note that by Lemma 2.2 and $\left[22\right.$, Tables], $D^{(5,3)} \cong S^{(5,3)}$ is a composition factor of $D^{\lambda} \downarrow_{\mathrm{s}_{8}}$. Let $\chi$ be the character of $S^{(5,3)}$. Let $y:=(2,6,8,3,4)$. In order to prove that $x_{3,1^{2}} D^{\lambda} \neq 0$ it is enough to prove that $\chi\left(y x_{3,1^{2}}\right) \neq 0$. Note that $y x_{3,1^{2}}=X_{+}-X_{-}$where

$$
\begin{aligned}
& X_{+}=y \sum_{g \in \mathrm{A}_{4,2^{2}}} \sum_{h \in \mathrm{S}_{\{2,6,8\}}} g h(2,6,8,3,4) h^{-1} g^{-1}, \\
& X_{-}=y \sum_{g \in \mathrm{S}_{4,2^{2}} \backslash \mathrm{A}_{4,2^{2}}} \sum_{h \in \mathrm{S}_{\{2,6,8\}}} g h(2,6,8,3,4) h^{-1} g^{-1} .
\end{aligned}
$$

It can be computed with GAP 18 that the number of elements appearing $X_{ \pm}$ corresponding to each conjugacy class of $\mathrm{S}_{8}$ is as follows $\left(X_{ \pm} \in F \mathrm{~A}_{8}\right.$ so that not all conjugacy classes have to be considered):

\begin{tabular}{l|c|c|c|c|c|c|c} 
cycle type & $\left(1^{8}\right)$ & $\left(2^{2}, 1^{4}\right)$ & $\left(2^{4}\right)$ & $\left(3,1^{5}\right)$ & $\left(3,2^{2}, 1\right)$ & $\left(3^{2}, 1^{2}\right)$ \\
\hline$X_{+}$ & 0 & 18 & 0 & 15 & 32 & 11 \\
$X_{-}$ & 2 & 13 & 0 & 10 & 12 & 46 \\
cycle type & $\left(4,2,1^{2}\right)$ & $\left(4^{2}\right)$ & $\left(5,1^{3}\right)$ & $(5,3)$ & $(6,2)$ & $(7,1)$ \\
\hline$X_{+}$ & 27 & 4 & 53 & 22 & 8 & 98 \\
$X_{-}$ & 67 & 24 & 36 & 12 & 18 & 48,
\end{tabular}

from which it easily follows that $\chi\left(y x_{3,1^{2}}\right) \equiv 2 \bmod 3$.

Lemma 3.10. Let $p=3, n \geq 8$ and $\lambda \in \mathscr{R} \mathscr{P}_{3}(n) \backslash\left\{\beta_{n}\right\}$. If $V$ is an irreducible spin representation of $G \in\left\{\widetilde{\mathrm{S}}_{n}, \widetilde{\mathrm{A}}_{n}\right\}$ indexed by $\lambda$ then there exists $\psi \in$ $\operatorname{Hom}_{G}\left(M_{3,1^{2}}, \operatorname{End}_{F}(V)\right)$ which does not vanish on $S_{3,1^{2}}$.

Proof. Assume first that $G=\widetilde{\mathrm{S}}_{n}$. By Lemma 3.4 there exists a composition factor of $D(\lambda, \delta) \downarrow_{\widetilde{S}_{8}}$ of the form $D(\mu, \varepsilon)$ with $\mu \in\{(5,2,1),(4,3,1)\}$. Let $\chi$ be the character of $D(\mu, \varepsilon)$ and $\chi^{(8), \pm}, \chi^{(6,2), 0}$ and $\chi^{(7,1), 0}$ be the characters of the reduction modulo 3 of the simple spin modules in characteristic 0 indexed by the corresponding partitions. Then $\chi \in\left\{1 / 2 \chi^{(6,2), 0}-\chi^{(8), \pm}, \chi^{(7,1), 0}\right\}$ using decomposition matrices and Lemma 2.17 . 
In order to prove the lemma for $\widetilde{\mathrm{S}}_{n}$ it is enough by Lemma 3.2 to prove that $x_{3,1^{2}} D(\mu, \varepsilon) \neq 0$. Let $y:=(2, \widetilde{6,8,3}, 4)$ and

$$
\begin{aligned}
& X_{+}=y \sum_{g \in \mathrm{A}_{4,2^{2}}} \sum_{h \in \mathrm{S}_{\{2,6,8\}}} \tilde{g} \widetilde{h}(2, \widetilde{6,8,3}, 4)(\widetilde{h})^{-1}(\widetilde{g})^{-1}, \\
& X_{-}=y \sum_{g \in \mathrm{S}_{4,2^{2}} \backslash \mathrm{A}_{4,2^{2}}} \sum_{h \in \mathrm{S}_{\{2,6,8\}}} \widetilde{g} \widetilde{h}(2, \widetilde{6,8,3}, 4)(\widetilde{h})^{-1}(\widetilde{g})^{-1} .
\end{aligned}
$$

Note that $y x_{3,1^{2}}=X_{+}-X_{-}$. It can be computed that the number of elements appearing $X_{ \pm}$corresponding to each conjugacy class of $\widetilde{\mathrm{S}}_{8}$ is as follows:

\begin{tabular}{l|c|c|c|c|c|c} 
cycle type & $\left(1^{8}\right)$ & $\left(1^{8}\right)$ & $\left(3,1^{5}\right)$ & $\left(3,1^{5}\right)$ & $\left(3^{2}, 1^{2}\right)$ & $\left(3^{2}, 1^{2}\right)$ \\
order of el. & 1 & 2 & 3 & 6 & 3 & 6 \\
\hline$X_{+}$ & 0 & 0 & 4 & 11 & 7 & 4 \\
$X_{-}$ & 2 & 0 & 4 & 6 & 32 & 14
\end{tabular}

\begin{tabular}{l|c|c|c|c|c|c|c} 
cycle type & $\left(5,1^{3}\right)$ & $\left(5,1^{3}\right)$ & $(5,3)$ & $(5,3)$ & $(7,1)$ & $(7,1)$ & others \\
order of el. & 5 & 10 & 15 & 30 & 7 & 14 & \\
\hline$X_{+}$ & 11 & 42 & 22 & 0 & 62 & 36 & 89 \\
$X_{-}$ & 9 & 27 & 12 & 0 & 42 & 6 & 134.
\end{tabular}

Since $X_{ \pm} \in F \widetilde{\mathrm{A}}_{8}$, it easily follows that $\chi\left(y x_{3,1^{2}}\right) \equiv 1 \bmod 3$. So the lemma holds for $\widetilde{\mathrm{S}}_{n}$. Assume now that $G=\widetilde{\mathrm{A}}_{n}$. If $V \cong E(\lambda, 0)$ then $V \cong D(\lambda, \pm) \downarrow \widetilde{\mathrm{A}}_{n}$. So in this case the lemma holds by the previous part. If $V \cong E(\lambda, \pm)$ the lemma can be proved similarly to Lemma 3.7 .

Lemma 3.11. Let $p \geq 3, n \geq 6, \lambda \in \mathscr{P}_{p}(n) \backslash \mathscr{H}_{p}(n)$ and $G \in\left\{\mathrm{S}_{n}, \mathrm{~A}_{n}\right\}$. Let $V$ be a $G$-module indexed by $\lambda$. Then there exists a non-zero $\psi \in \operatorname{Hom}_{G}\left(S_{1^{3}}, \operatorname{End}_{F}(V)\right)$. If $p \neq 3$ then $\psi$ extends to $\varphi \in \operatorname{Hom}_{G}\left(M_{1^{3}}, \operatorname{End}_{F}(V)\right)$.

Proof. By Lemma 3.3 it is enough to prove that $x_{1^{3}} V \neq 0$. We will consider $x_{1^{3}}$ as an element of $F \mathrm{~A}_{n}$. By Lemma 3.6 it is enough to prove that $x_{1^{3}} E \neq 0$ for all irreducible modules $E$ of $\mathrm{A}_{6}$ indexed by $\mu \in \mathscr{P}_{p}(6) \backslash \mathscr{H}_{p}(6)$. So we may assume that $E \in\left\{E^{(4,2)}, E^{\left(3^{2}\right)}, E_{ \pm}^{(3,2,1)}\right\}$ if $p>5, E \in\left\{E^{(4,2)}, E^{\left(3^{2}\right)}\right\}$ if $p=5$ or $E=E^{(4,2)}$ if $p=3$. Each of these modules is just the reduction modulo $p$ of the characteristic 0 module indexed by the same partition, so that characters are known.

Note that $x_{1^{3}} E \neq 0$ if and only if $x_{1^{3}}(1,2,3) E \neq 0$. It can be computed that $\pm x_{1^{3}}(1,2,3)$ is equal to

$$
(1,3)(2,4)+(1,2)(3,4)+(1,4)(2,3)+1-(1,4,3)-(1,2,4)-(2,3,4)-(1,3,2) .
$$

If $\chi$ is the character of $E$ it then follows that $\chi\left(x_{1^{3}}(1,2,3)\right)= \pm 12 \not \equiv 0 \bmod p$ if $p \geq 5$. So assume that $p=3$. It can be computed that $\pm x_{1^{3}}(2,6,3,5,4)$ is equal to

$$
\begin{aligned}
& (2,5,4,6,3)+(1,2,6,3)(4,5)+(1,6,3,5,4)+(1,5,4,2)(3,6) \\
& -(4,6)(4,5)-(1,5,4)(2,6,3)-(1,2)(3,5,4,6)-(1,6,3)(2,5,4)
\end{aligned}
$$

and so $\chi\left(x_{1^{3}}(2,6,3,5,4)\right)= \pm 2 \not \equiv 0 \bmod 3$. The lemma then follows.

Lemma 3.12. Let $p \geq 3, n \geq 4, G \in\left\{\widetilde{\mathrm{S}}_{n}, \widetilde{\mathrm{A}}_{n}\right\}$ and $\lambda \in \mathscr{R} \mathscr{P}_{p}(n) \backslash\left\{\beta_{n}\right\}$. If $V$ is a spin irreducible representation of $G$ indexed by $\lambda$ then there exists a non-zero $\psi \in \operatorname{Hom}_{G}\left(S_{1^{3}}, \operatorname{End}_{F}(V)\right)$. If $p \neq 3$ then $\psi$ extends to $\varphi \in \operatorname{Hom}_{G}\left(M_{1^{3}}, \operatorname{End}_{F}(V)\right)$. 
Proof. From [34, Lemma 2.4] we have that if $m \geq 6$ and $\mu \in \mathscr{R} \mathscr{P}_{p}(m) \backslash\left\{\beta_{m}\right\}$, then $D(\mu) \downarrow_{\widetilde{\mathrm{s}}_{m-1}}$ has a composition factor which is not basic spin.

Assume first that $p \geq 5$. In this case it can then be easily checked that $V \downarrow_{\tilde{A}_{4}}$ has a composition factor $E((3,1), \pm)$. Let $g:=\widetilde{(1,2,3)}$. Up to exchange of $C_{3}^{ \pm}$we have that

$$
\begin{aligned}
g x_{1^{3}}=1 & +z^{\cdots}\left(\widetilde{(1,2)(3,4)}+z^{\cdots}(\widetilde{1,3)(2,4})+z^{\cdots(1, \widetilde{(1,4)}(2,3)}\right. \\
& -z(\widetilde{(1,4,3)}-z(\widetilde{1,2,4})-z(\widetilde{2,3,4})-(\widetilde{1,3,2}) .
\end{aligned}
$$

Since $p \geq 5, E((3,1)) \cong S((3,1))$ by Lemma 2.17. If $\chi$ is the character of $E((3,1))$ we then have that $\chi\left(g x_{1^{3}}\right)= \pm 6$ and so the action of $x_{1^{3}}$ on at least one between $E((3,1),+)$ and $E((3,1),-1)$ is non-zero.

If $G=\widetilde{\mathrm{S}}_{n}$ both $E((3,1),+)$ and $E((3,1),-)$ are composition factors of $V \downarrow_{\widetilde{\AA}_{4}}$, so by Lemma 3.3 there exists a non-zero $\psi \in \operatorname{Hom}_{G}\left(S_{1^{3}}, \operatorname{End}_{F}(V)\right)$.

If $G=\widetilde{\mathrm{A}}_{n}$ and $V=E(\lambda, 0)$ then $V=D(\lambda, \pm) \downarrow_{\widetilde{\mathrm{A}}_{n}}$, so again both $E((3,1),+)$ and $E((3,1),-)$ are composition factors of $V \downarrow_{\widetilde{A}_{4}}$ and we can conclude as in the previous case.

If $G=\widetilde{\mathrm{A}}_{n}$ and $V=E(\lambda, \pm)$ then $E((3,1), \pm)$ is a composition factor of $E(\lambda,+) \downarrow_{\widetilde{\mathrm{A}}_{4}}$ if and only if $E((3,1), \mp)$ is a composition factor of $E(\lambda,-) \downarrow_{\widetilde{\mathrm{A}}_{4}}$, so by Lemma 3.3 there exists a non-zero $\psi_{+} \in \operatorname{Hom}_{\widetilde{\mathrm{A}}_{n}}\left(S_{1^{3}}, \operatorname{End}_{F}(E(\lambda,+))\right)$ or a non-zero $\psi_{-} \in$ $\operatorname{Hom}_{\widetilde{\mathrm{A}}_{n}}\left(S_{1^{3}}, \operatorname{End}_{F}(E(\lambda,-))\right)$. Since $S_{1^{3}} \cong S_{1^{3}}^{\sigma}$ and $E(\lambda, \pm) \cong E(\lambda, \mp)^{\sigma}$ for $\sigma \in$ $\mathrm{S}_{n} \backslash \mathrm{A}_{n}$, it follows that there exists a non-zero $\left.\psi_{+} \in \operatorname{Hom}_{\widetilde{\mathrm{A}}_{n}}\left(S_{1^{3}}, \operatorname{End}_{F}(V)\right)\right)$.

Assume now that $p=3$. Then $n \geq 5$ and $V \downarrow_{\widetilde{A}_{5}}$ has a composition factor $E((4,1), 0)$. Let $g:=\left(\widetilde{1,2)(4,5)}\right.$. Then, up to exchange of $C_{3}^{ \pm}$,

$$
\begin{aligned}
g x_{1^{3}}= & (1, \widetilde{2,3,5}, 4)+(1, \widetilde{5,4,3}, 2)+z(\widetilde{2,5,4})+z^{\cdots}(\widetilde{1,3)(4,5)} \\
& -z(1, \widetilde{2,5,4}, 3)-z(1, \widehat{3,5,4}, 2)-(\widetilde{1,5,4})-z^{\cdots}(2, \widetilde{3,3)(4,5)} .
\end{aligned}
$$

By Lemma 2.17 and using decomposition matrices, $E((4,1)) \cong S((4,1))$. If $\chi$ is the character of $E((4,1), 0)$ then $\chi\left(g x_{1^{3}}\right)= \pm 4$, from which the lemma follows also in this case by Lemma 3.3 .

Lemma 3.13. Let $n \geq 6$ and $G \in\left\{\mathrm{S}_{n}, \mathrm{~A}_{n}\right\}$. Assume that $p \geq 5$ and $\lambda \in \mathscr{P}_{p}(n) \backslash$ $\mathscr{H}_{p}(n)$ with $h(\lambda), h\left(\lambda^{\mathrm{M}}\right) \geq 3$ or that $p=3$ and $\lambda \in \mathscr{P}_{3}(n)$ with $h(\lambda), h\left(\lambda^{\mathrm{M}}\right) \geq 4$. Let $V$ be a $G$-module indexed by $\lambda$. Then there exists a non-zero $\psi \in \operatorname{Hom}_{G}\left(S_{1^{5}}, \operatorname{End}_{F}(V)\right)$. If $p \neq 5$ then $\psi$ extends to $\varphi \in \operatorname{Hom}_{G}\left(M_{1^{5}}, \operatorname{End}_{F}(V)\right)$.

Proof. If $p \geq 5$ and $n \geq 9$ then by Lemma 3.6 there exists $\mu \in \mathscr{P}_{p}(9) \backslash \mathscr{H}_{p}(9)$ with $h(\mu), h\left(\mu^{\mathrm{M}}\right) \geq 3$ such that $D^{\mu}$ is a composition factor of $D^{\lambda} \downarrow_{\mathrm{S}_{9}}$. It can then be easily checked using Lemma 2.2 and decomposition matrices that if $p \geq 7$ then $D^{(3,2,1)}$ is a composition factor of $D^{\lambda} \downarrow_{S_{6}}$, while if $p=5$ then $n \geq 7$ and $E^{(4,2,1)}$ is a composition factor of $D^{\lambda} \downarrow_{A_{7}}$. If $p=3$ then $n \geq 8$ and by [38, Lemma 4.13], $D^{\left(4,2,1^{2}\right)}$ is a composition factor of $D^{\lambda} \downarrow_{S_{8}}$. Each of these modules is just the reduction modulo $p$ of the characteristic 0 module indexed by the same partition (for example looking at decomposition matrices), so characters are known.

Consider $x_{1^{5}}$ and $C_{5}^{ \pm}$upon projection to $\mathrm{A}_{n}$. 
If $p \geq 7$ let $g:=(1,2,3,4,5)$. Then, up to exchange of $C_{5}^{ \pm}$, we have that the number of elements of $C_{5}^{ \pm} g$ in each conjugacy class of $\mathrm{S}_{6}$ is as follows:

\begin{tabular}{l|c|c|c|c|c|c|c} 
cycle type & $\left(1^{6}\right)$ & $\left(2^{2}, 1^{2}\right)$ & $\left(3,1^{3}\right)$ & $\left(3^{2}\right)$ & $(4,2)$ & $(5,1)$ & others \\
\hline$C_{5}^{+} g$ & 1 & 10 & 5 & 5 & 20 & 31 & 0 \\
$C_{5}^{-} g$ & 0 & 10 & 10 & 10 & 20 & 22 & 0.
\end{tabular}

If $\chi$ is the character of $D^{(3,2,1)}$ it then follows that $\chi\left(x_{15} g\right)= \pm 45$.

If $p=5$ let $g:=(2,7,4)(3,6,5)$. Then, up to exchange of $C_{5}^{ \pm}$, we have that the number of elements of $C_{5}^{ \pm} g$ in each conjugacy class of $\mathrm{S}_{7}$ is as follows:

\begin{tabular}{l|c|c|c|c|c|c|c|c} 
cycle type & $\left(2^{2}, 1^{3}\right)$ & $\left(3,1^{4}\right)$ & $\left(3,2^{2}\right)$ & $\left(3^{2}, 1\right)$ & $(4,2,1)$ & $\left(5,1^{2}\right)$ & $(7)$ & others \\
\hline$C_{5}^{+} g$ & 0 & 3 & 6 & 3 & 27 & 9 & 24 & 0 \\
$C_{5}^{-} g$ & 3 & 0 & 12 & 6 & 9 & 18 & 24 & 0.
\end{tabular}

If $\chi$ is the character of $E^{(4,2,1)}$ it then follows that $\chi\left(x_{1^{5}} g\right)= \pm 9$.

If $p=3$ and $h(\lambda), h\left(\lambda^{\mathrm{M}}\right) \geq 4$ let $g=(1,2,3,4,5)(6,7,8)$. Then, up to exchange of $C_{5}^{ \pm}$, we have that the number of elements of $C_{5}^{ \pm} g$ in each conjugacy class of $\mathrm{S}_{8}$ is as follows:

\begin{tabular}{|c|c|c|c|c|c|c|}
\hline cycle type & $\left(3,1^{5}\right)$ & $\left(3,2^{2}, 1\right)$ & $\left(3^{2}, 1^{2}\right)$ & \multicolumn{2}{|c|}{$\left(4,2,1^{2}\right)$} & $\left(4^{2}\right)$ \\
\hline$C_{5}^{+} g$ & 0 & 5 & 5 & \multicolumn{2}{|c|}{5} & 10 \\
\hline$C_{5}^{-} g$ & 1 & 0 & 5 & \multicolumn{2}{|c|}{10} & 5 \\
\hline \multicolumn{2}{|c|}{ cycle type } & $(5,3)$ & $(6,2)$ & \multicolumn{3}{|c|}{ others } \\
\hline$C_{5}^{+} g$ & 5 & 12 & 10 & 20 & 0 & \\
\hline$C_{5}^{-} g$ & 0 & 11 & 15 & 25 & 0 & \\
\hline
\end{tabular}

If $\chi$ is the character of $D^{\left(4,2,1^{2}\right)}$ it can be easily checked that $\chi\left(x_{1^{5}} g\right)= \pm 5$.

For $S_{n}$ the lemma then follows. For $A_{n}$ it holds similarly to Lemma 3.7 .

Lemma 3.14. Let $p \geq 3, n \geq 6, G \in\left\{\widetilde{\mathrm{S}}_{n}, \widetilde{\mathrm{A}}_{n}\right\}$ and $\lambda \in \mathscr{R} \mathscr{P}_{p}(n) \backslash\left\{\beta_{n}\right\}$ with $\lambda_{1} \geq 5$. If $V$ is an irreducible spin representation of $G$ indexed by $\lambda$, then there exists a non-zero $\psi \in \operatorname{Hom}_{G}\left(S_{1^{5}}, \operatorname{End}_{F}(V)\right)$. If $p \neq 5$ then $\psi$ extends to $\varphi \in$ $\operatorname{Hom}_{G}\left(M_{1^{5}}, \operatorname{End}_{F}(D)\right)$.

Proof. If $p \geq 7$ and $n \geq 11$ then by Lemma 3.4 there exists $\mu \in \mathscr{R} \mathscr{P}_{p}(11) \backslash\left\{\beta_{11}\right\}$ such that $D(\mu)$ is a composition factor of $D(\lambda) \downarrow_{\widetilde{S}_{11}}$. It can then be easily checked using Lemma 2.7 that $D(\lambda) \downarrow_{\widetilde{\mathrm{S}}_{6}}$ has a composition factor $D((5,1), 0)$. Let $g=$ $(1, \widehat{2,3,4}, 5)$. Up to exchange of $C_{5}^{ \pm}$, we have that the number of elements of $C_{5}^{ \pm} g$ in each conjugacy class of $\widetilde{\mathrm{S}}_{6}$ is as follows:

\begin{tabular}{l|c|c|c|c|c|c|c|c|c} 
cycle type & $\left(1^{6}\right)$ & $\left(1^{6}\right)$ & $\left(3,1^{3}\right)$ & $\left(3,1^{3}\right)$ & $\left(3^{2}\right)$ & $\left(3^{2}\right)$ & $(5,1)$ & $(5,1)$ & others \\
order of el. & 1 & 2 & 3 & 6 & 3 & 6 & 5 & 10 & \\
\hline$C_{5}^{+} g$ & 0 & 0 & 5 & 5 & 5 & 5 & 2 & 20 & 30 \\
$C_{5}^{-} g$ & 1 & 0 & 0 & 5 & 0 & 5 & 11 & 20 & 30.
\end{tabular}

Let $\chi$ be the character of $D((5,1), 0) \cong S((5,1), 0)$ (by Lemma 2.17 since $p>6$ ). Then $\chi\left(x_{15} g\right)= \pm 45 \neq 0$.

Assume next that $p=3$ or $p=5$ and $\lambda_{1} \geq 6$. Then $n \geq 7$. If $p=3$ then $E((5,2), 0)$ is a composition factor of $V \downarrow_{\widetilde{A}_{7}}$ by Lemma 2.7 by always removing the bottom normal node for which the obtained partition is in $\mathscr{R} \mathscr{P}_{p}(m)$. If $p=5$ and $\lambda_{1} \geq 6$ then similarly $E((6,1), 0)$ is a composition factor of $V \downarrow_{\widetilde{A}_{7}}$. Again by Lemma 2.17 and decomposition matrices, $E((5,2), 0)$ for $p=3$ and $E((6,1), 0)$ for $p=5$ 
are just the reduction modulo $p$ of the corresponding characteristic 0 modules. Let $g=(2,3) \widetilde{(4,5,6}, 7)$. Up to exchange of $C_{5}^{ \pm}$and choice of $g$, we have that the number of elements of $C_{5}^{ \pm} g$ in each conjugacy class of $\widetilde{\mathrm{S}}_{7}$ is as follows:

\begin{tabular}{l|c|c|c|c|c|c} 
cycle type & $\left(1^{7}\right)$ & $\left(1^{7}\right)$ & $\left(3,1^{4}\right)$ & $\left(3,1^{4}\right)$ & $\left(3^{2}, 1\right)$ & $\left(3^{2}, 1\right)$ \\
order of el. & 1 & 2 & 3 & 6 & 3 & 6 \\
\hline$C_{5}^{+} g$ & 0 & 0 & 1 & 0 & 5 & 4 \\
$C_{5}^{-} g$ & 0 & 0 & 0 & 1 & 4 & 5 \\
\multicolumn{2}{c}{ cycle type } & $\left(5,1^{2}\right)$ & $\left(5,1^{2}\right)$ & $(7)$ & $(7)$ & others \\
order of el. & 5 & 10 & 7 & 14 & \\
\hline$C_{5}^{+} g$ & 6 & 5 & 14 & 10 & 27 \\
$C_{5}^{-} g$ & 5 & 6 & 10 & 14 & 27.
\end{tabular}

If $p=3$ and $\chi$ is the character of $E((5,2), 0)$ then $\chi\left(x_{1^{5}} g\right)= \pm 10$. If $p=5$ and $\chi$ is the character of $E((6,1), 0)$ then $\chi\left(x_{1^{5}} g\right)= \pm 18$.

Last assume that $p=5$ and $\lambda_{1}=5$. Then $n \geq 8$. If $n \geq 11$ and $D(\lambda) \downarrow_{\widetilde{S}_{11}}$ has a composition factor $D(\mu)$ with $\mu_{1} \geq 6$ we can apply the previous paragraph. So we may assume this is not the case. Then by Lemma 3.4 if $n \geq 11$ then $D(\lambda) \downarrow_{\widetilde{S}_{11}}$ has a composition factor $D((5,3,2,1))$ or $D((5,4,2))$. It can then be checked (also when $n \leq 10$ ) that $D((5,2,1), 0)$ is a composition factor of $D(\lambda) \downarrow_{\widetilde{S}_{8}}$. Let $g:=(2,3) \widetilde{(4,5,7})(6,8)$. Up to exchange of $C_{5}^{ \pm}$and choice of $g$, we have that the number of elements of $C_{5}^{ \pm} g$ in each conjugacy class of $\widetilde{\mathrm{S}}_{8}$ is as follows:

\begin{tabular}{l|c|c|c|c|c|c} 
cycle type & $\left(1^{8}\right)$ & $\left(1^{8}\right)$ & $\left(3,1^{5}\right)$ & $\left(3,1^{5}\right)$ & $\left(3^{2}, 1^{2}\right)$ & $\left(3^{2}, 1^{2}\right)$ \\
order of el. & 1 & 2 & 3 & 6 & 3 & 6 \\
\hline$C_{5}^{+} g$ & 0 & 0 & 0 & 0 & 2 & 0 \\
$C_{5}^{-} g$ & 0 & 0 & 0 & 0 & 0 & 2
\end{tabular}

\begin{tabular}{l|c|c|c|c|c|c|c} 
cycle type & $\left(5,1^{3}\right)$ & $\left(5,1^{3}\right)$ & $(5,3)$ & $(5,3)$ & $(7,1)$ & $(7,1)$ & others \\
order of el. & 5 & 10 & 15 & 30 & 7 & 14 & \\
\hline$C_{5}^{+} g$ & 0 & 2 & 2 & 8 & 10 & 12 & 36 \\
$C_{5}^{-} g$ & 2 & 0 & 8 & 2 & 12 & 10 & 36.
\end{tabular}

If $\chi$ is the character of $D((5,2,1), 0)$ then $\chi\left(x_{1^{5}} g\right)= \pm 4$ (using decomposition matrices and Lemma 2.17 it can be checked that $D((5,2,1), 0)$ is the reduction modulo 5 of either module indexed by $(5,2,1)$ in characteristic 0$)$. 3.7.

The lemma then follows for $\widetilde{\mathrm{S}}_{n}$. For $\widetilde{\mathrm{A}}_{n}$ it follows similarly to the proof of Lemma

In the next section we will study the structure of certain permutation modules. In $\$ 5.1$ to $\$ 5.3$ we will then study more in detail most classes of modules for which some of the results in this section do not apply and obtain similar results on the endomorphisms rings of those modules. These results will then be used in $\$ 6.1$ to 6.5 to study tensor products of certain special classes of modules.

\section{Permutation modules}

In order to extend the results obtained in the previous section to (some) of the classes of families which were not considered, we will need to study permutation modules more in detail and then study restrictions of some classes of irreducible 
modules to certain subgroups. We start here by considering the structure of certain permutation modules.

The following three lemmas on the structure of $M^{\lambda}$ for certain 2-row partitions $\lambda$ follow easily from [22, 17.17,24.15] and [24, 6.1.21,2.7.41].

Lemma 4.1. Let $1 \leq k<p$. Then $M_{k} \sim S_{k} \mid M_{k-1}$.

Lemma 4.2. Let $p \geq 3$ and $n \geq 2$. If $p \nmid n$ then $M_{1} \cong D_{0} \oplus D_{1}$, while if $p \mid n$ then $M^{(n-1,1)} \cong D_{0}\left|D_{1}\right| D_{0}$.

Lemma 4.3. Let $p=3$ and $n \geq 4$. Then

$$
M_{2} \cong\left\{\begin{array}{lll}
M_{1} \oplus D_{2}, & n \equiv 0 & \bmod 3 \\
D_{1} \oplus\left(D_{0}\left|D_{2}\right| D_{0}\right), & n \equiv 1 \bmod 3 \\
D_{0} \oplus\left(D_{1}\left|D_{2}\right| D_{1}\right), & n \equiv 2 & \bmod 3
\end{array}\right.
$$

We will also need information about the structure of certain permutation modules corresponding to subgroups $\mathrm{S}_{n-k}$.

Lemma 4.4. Let $p \geq 3$ and $n \not \equiv 0 \bmod p$. If $n \geq 2$ then

$$
M_{1} \cong D_{1} \oplus M_{0}
$$

If $n \geq 4$ then

$$
M_{1^{2}} \oplus M_{0} \cong D_{1^{2}} \oplus M_{2} \oplus M_{1}
$$

If $p \geq 5$ and $n \geq 6$ then

$$
M_{1^{3}} \oplus M_{3} \oplus M_{2} \oplus M_{1} \cong D_{1^{3}} \oplus M_{2,1}^{\oplus 2} \oplus M_{1^{2}} \oplus M_{0} .
$$

Proof. From Lemma 2.23 we have that in each of the above cases $D_{1_{k}} \cong S_{1_{k}} \subseteq M_{1^{k}}$. Since $M_{1^{k}}$ and $D_{1^{k}}$ are self-dual, $D_{1^{k}}$ is also a quotient of $M_{1^{k}}$. Let $V \subseteq M_{1^{k}}$ with $V \cong D_{1^{k}}$ and $W \subseteq M_{1^{k}}$ with $M_{1^{k}} / W \cong D_{1^{k}}$. By [22, 12.1] we have that $\left[M_{1^{k}}\right.$ : $\left.D_{1^{k}}\right]=1$, so $V \nsubseteq W$. By dimension we then have that $M_{1^{k}}=V \oplus W$, so $D_{1^{k}} \cong S_{1^{k}}$ is a direct summand of $M_{1^{k}}$. The lemma then follows by comparing composition factors (for example using Specht filtrations from [22, 17.14]) and Lemma 2.21] since if $\lambda \unrhd\left(n-k, 1^{k}\right)$ and $k<p$ then $\lambda \in \mathscr{P}_{p}(n)$.

Lemma 4.5. Let $p \geq 3$ and $n \equiv 0 \bmod p$. If $n \geq 2$ then

$$
M_{1} \cong Y_{1}
$$

and if $n \geq 4$

$$
M_{1^{2}} \cong M_{2} \oplus Y_{2}
$$

If $p \geq 5$ and $n \geq 6$ then

$$
M_{1^{3}} \oplus M_{3} \cong M_{2,1}^{\oplus 2} \oplus Y_{3}
$$

and if $n \geq 8$

$$
M_{1^{4}} \oplus M_{2^{2}} \oplus M_{3,1}^{\oplus 2} \cong M_{2,1^{2}}^{\oplus 2} \oplus M_{4} \oplus Y_{4} .
$$

If $p=3$ and $n \geq 6$ then

$$
M_{1^{3}} \oplus M_{1} \cong M_{2,1} \oplus M_{1^{2}} \oplus Y_{3}^{\prime} .
$$


In each of the above cases $Y_{k}$ or $Y_{k}^{\prime}$ is indecomposable with simple head and socle isomorphic to $D_{1^{k-1}}$ and

$$
\begin{aligned}
& Y_{1} \cong \overbrace{D_{0}\left|D_{1}\right|}^{S_{1}} \overbrace{\overbrace{D_{0}}}^{S_{0}}, \\
& Y_{2} \sim \overbrace{D_{1} \mid D_{1^{2}}}^{S_{1^{2}}} \mid \overbrace{D_{0} \mid D_{1}}^{S_{1}}, \\
& Y_{3} \sim \overbrace{D_{1^{2}} \mid D_{1^{3}}}^{S_{1^{3}}} \mid \overbrace{D_{1} \mid D_{1^{2}}}^{S_{1^{2}}}, \\
& Y_{3}^{\prime} \sim{S_{1^{3}}\left|S_{2,1}\right| S_{1^{2}},}_{Y_{4}}^{S_{S_{1^{4}}}|\overbrace{D_{13}}| \overbrace{D_{1^{2}} \mid D_{1^{3}}}^{S_{1^{3}}}} .
\end{aligned}
$$

Proof. Note that $M_{1^{k}}=M^{\left(n-k, 1^{k-1}\right) \uparrow \mathrm{S}_{n}}$. In particular in each of the above cases since $\left(n-k, 1^{k-1}\right) \in \mathscr{P}_{p}(n-1)$ from Lemmas 2.1 and 2.23 and self-duality of $M^{\left(n-k, 1^{k-1}\right)}$ we have that $D^{\left(n-k, 1^{k-1}\right)} \cong S^{\left(n-k, 1^{k-1}\right)}$ and that $e_{-k} D^{\left(n-k, 1^{k-1}\right) \uparrow \mathrm{S}_{n}}$ is a direct summand of $M_{1 k}$. Let $Y_{k}$ or $Y_{k}^{\prime}$ be this direct summand. Then $Y_{k}$ or $Y_{k}^{\prime}$ has simple head and socle isomorphic to $D_{1^{k-1}}$ by Lemma 2.2 and it has the right Specht filtration by [22, Corollary 17.14] and block decomposition. Structure of hook Specht modules can be obtained by Lemma 2.23 .

The lemma then follows by comparing composition factors (for example using Specht filtrations) and Lemma 2.21, since $\lambda \in \mathscr{P}_{p}(n)$ if $\lambda \triangleright\left(n-k, 1^{k}\right)$ and $k \leq p$.

\section{More ON ENDOMORPHISMS RINGS}

In this section we study branching for certain classes of modules in order to extend in many cases results from $\$ 3.3$ to families of modules which were not considered there. We divide this section according to different classes of modules.

\subsection{Partitions with two or three rows.}

Lemma 5.1. Let $p=3, n \geq 7, G \in\left\{\mathrm{S}_{n}, \mathrm{~A}_{n}\right\}$ and $\lambda=(n-2,2)$. Let $V$ be an irreducible $F G$-module indexed by $\lambda$. If $n \not \equiv 2 \bmod 3$ then there exists $\psi \in$ $\operatorname{Hom}_{H}\left(M_{3}, \operatorname{End}_{F}(V)\right)$ which does not vanish on $S_{3}$.

Proof. By [33, Lemma 1.8], $\lambda \neq \lambda^{\mathrm{M}}$, so $V \cong D^{\lambda}=D_{2}$ or $E^{\lambda}$. So it is enough to prove the lemma for $S_{n}$. From [38, Lemma 6.5] it is enough to prove that

$$
\operatorname{dim} \operatorname{End}_{\mathrm{S}_{n-3,3}}\left(D_{2} \downarrow_{\mathrm{S}_{n-3,3}}\right)>\operatorname{dim} \operatorname{End}_{\mathrm{S}_{n-2,2}}\left(D_{2} \downarrow_{\mathrm{S}_{n-2,2}}\right) .
$$

Note that the assumption on $n$ is equivalent to $(n-2,2)$ not being a JS-partition.

If the two removable nodes have different residue this holds by [38, Lemma 6.7]. So we may assume that the removable nodes have the same residue, in which case 
$n \equiv 0 \bmod 3$. From Mackey induction-reduction theorem we have that

$$
\begin{aligned}
& M_{1} \downarrow_{\mathrm{S}_{n-2,2}} \cong 1 \uparrow_{\mathrm{S}_{n-2,1^{2}}}^{\mathrm{S}_{n-2,2}} \oplus 1 \uparrow \mathrm{S}_{n-3,1,2}^{\mathrm{S}_{n-2,2}} \\
& \cong\left(M^{(n-2)} \otimes M^{\left(1^{2}\right)}\right) \oplus\left(M^{(n-3,1)} \otimes M^{(2)}\right), \\
& M_{1} \downarrow_{\mathrm{S}_{n-3,3}} \cong 1 \uparrow \mathrm{S}_{n-3,2,1}^{\mathrm{S}_{n-3,3}} \oplus 1 \uparrow \mathrm{S}_{n-4,1,3}^{\mathrm{S}_{n-3}} \\
& \cong\left(M^{(n-3)} \otimes M^{(2,1)}\right) \oplus\left(M^{(n-4,1)} \otimes M^{(3)}\right), \\
& M_{2 \downarrow} \mathrm{S}_{n-2,2} \cong 1 \oplus 1 \uparrow \mathrm{S}_{n-3,13}^{\mathrm{S}_{n-2,2}} \oplus 1 \uparrow \mathrm{S}_{\mathrm{S}_{n-4,2}}^{\mathrm{S}_{n-2,2}} \\
& \cong\left(M^{(n-2)} \otimes M^{(2)}\right) \oplus\left(M^{(n-3,1)} \otimes M^{\left(1^{2}\right)}\right) \oplus\left(M^{(n-4,2)} \otimes M^{(2)}\right), \\
& M_{2} \downarrow \mathrm{S}_{n-3,3} \cong 1 \uparrow \mathrm{S}_{\mathrm{S}_{n-3,2,1}}^{\mathrm{S}_{n-3,3}} \oplus 1 \uparrow \mathrm{S}_{n-4,1,2,1}^{\mathrm{S}_{n-3,3}} \oplus 1 \uparrow \mathrm{S}_{n-5,2,3}^{\mathrm{S}_{n-3,3}} \\
& \cong\left(M^{(n-3)} \otimes M^{(2,1)}\right) \oplus\left(M^{(n-4,1)} \otimes M^{(2,1)}\right) \oplus\left(M^{(n-5,2)} \otimes M^{(3)}\right) \text {. }
\end{aligned}
$$

From Lemma 4.3 we have that $M_{2} \cong M_{1} \oplus D_{2}$. Comparing $M_{2} \downarrow_{H}$ and $M_{1} \downarrow_{H}$ for $H \in\left\{\mathrm{S}_{n-2,2}, \mathrm{~S}_{n-3,3}\right\}$ using Lemmas 4.2 and 4.3 , it follows that

$$
\begin{aligned}
& D_{2} \downarrow_{\mathrm{S}_{n-2,2}} \cong\left(D^{(n-3,1)} \otimes\left(D^{(2)} \oplus D^{\left(1^{2}\right)}\right)\right) \oplus\left(\left(D^{(n-2)}\left|D^{(n-4,2)}\right| D^{(n-2)}\right) \otimes D^{(2)}\right), \\
& D_{2} \downarrow_{\mathrm{S}_{n-3,3}} \sim\left(D^{(n-5,2)} \otimes D^{(3)}\right) \oplus\left(\left(D^{(n-3)}\left|D^{(n-4,1)}\right| D^{(n-3)}\right) \otimes\left(D^{(3)}\left|D^{(2,1)}\right| D^{(3)}\right)\right) .
\end{aligned}
$$

It then follows that

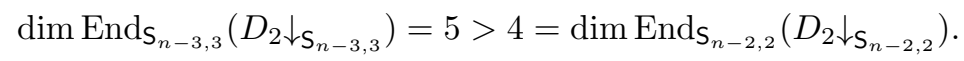

Lemma 5.2. Let $p \geq 3, n \geq 6$ with $n \not \equiv 0 \bmod p$ and $\lambda \in \mathscr{P}_{p}(n) \backslash \mathscr{H}_{p}(n)$. If $\lambda$ is not $J S$ then $\bar{D}_{0} \oplus \bar{D}_{1} \oplus \bar{D}_{3} \subseteq \operatorname{End}_{F}\left(D^{\lambda}\right)$.

Proof. Clearly $\bar{D}_{0} \cong D_{0} \subseteq \operatorname{End}_{F}\left(D^{\lambda}\right)$. From Lemmas 2.2 and 2.20 we have that

$$
\operatorname{dim} \operatorname{Hom}_{S_{n}}\left(M_{1}, \operatorname{End}_{F}\left(D^{\lambda}\right)\right)=\operatorname{dim}_{\operatorname{End}_{S_{n-1}}}\left(D^{\lambda}\right) \geq 2 .
$$

From Lemma 4.4 we then have that $\bar{D}_{1} \cong D_{1} \subseteq \operatorname{End}_{F}\left(D^{\lambda}\right)$. From Lemma 2.23 we have that $\bar{D}_{3} \cong S_{1^{3}}$. So $\bar{D}_{3} \subseteq \operatorname{End}_{F}\left(D^{\lambda}\right)$ by Lemma 3.11

Lemma 5.3. Let $p \geq 5, n \geq 6$ with $n \equiv 0 \bmod p$ and $\lambda \in \mathscr{P}_{p}(n)$. If $h(\lambda)=2$ and $\lambda_{1}-\lambda_{2} \not \equiv 0,-1,-2 \bmod p$ then $\bar{D}_{0} \oplus \bar{D}_{2} \subseteq \operatorname{End}_{F}\left(D^{\lambda}\right)$ or $\bar{D}_{0} \oplus \bar{D}_{1} \oplus \bar{D}_{3} \subseteq$ $\operatorname{End}_{F}\left(D^{\lambda}\right)$.

Proof. Notice that by Lemma 2.2 and considering branching in characteristic 0 ,

$$
\begin{aligned}
D^{\lambda} \downarrow_{S_{n-2,2}} \cong & \left(D^{\left(\lambda_{1}-2, \lambda_{2}\right)} \otimes D^{(2)}\right) \oplus\left(D^{\left(\lambda_{1}-1, \lambda_{2}-1\right)} \otimes D^{(2)}\right) \\
& \oplus\left(D^{\left(\lambda_{1}-1, \lambda_{2}-1\right)} \otimes D^{\left(1^{2}\right)}\right) \oplus\left(D^{\left(\lambda_{1}, \lambda_{2}-2\right)} \otimes D^{(2)}\right)^{\oplus a},
\end{aligned}
$$

with $a=1$ if $\lambda_{2} \geq 2$ and $\lambda_{1}-\lambda_{2} \not \equiv-3 \bmod p$ or $a=0$ else. From Lemmas 2.20 and 4.5 it follows that $\bar{D}_{1}$ or $\bar{D}_{2}$ is contained in $\operatorname{End}_{F}\left(D^{\lambda}\right)$. From Lemmas 2.23 and 3.11 we also have that $\bar{D}_{2}$ or $\bar{D}_{3}$ is contained in $\operatorname{End}_{F}\left(D^{\lambda}\right)$. The lemma follows.

Lemma 5.4. Let $p \geq 3, n \geq 6$ with $n \equiv 0 \bmod p$ and $\lambda \in \mathscr{P}_{p}(n)$. If $h(\lambda)=2$, $\lambda_{1}>\lambda_{2} \geq 2$ and $\lambda_{1}-\lambda_{2} \equiv 0$ or $-1 \bmod p$, then $\bar{D}_{0} \oplus \bar{D}_{2} \subseteq \operatorname{End}_{F}\left(D^{\lambda}\right)$ or $\bar{D}_{0} \oplus \bar{D}_{1} \oplus \bar{D}_{3} \subseteq \operatorname{End}_{F}\left(D^{\lambda}\right)$. 
Proof. We will use Lemma 2.2 without further reference to it.

We may assume that $\bar{D}_{2} \not \subset \operatorname{End}_{F}\left(D^{\lambda}\right)$. From Lemmas 2.23 and 3.11 we then have that $\bar{D}_{3} \subseteq \operatorname{End}_{F}\left(D^{\lambda}\right)$. So it is enough to prove that $\bar{D}_{1} \cong D_{1} \subseteq \operatorname{End}_{F}\left(D^{\lambda}\right)$. From Lemmas 2.20 and 4.5 it is enough to prove that

$$
\operatorname{dim} \operatorname{End}_{S_{n-2}}\left(D^{\lambda} \downarrow_{S_{n-2}}\right)-\operatorname{dim} \operatorname{End}_{S_{n-2,2}}\left(D^{\lambda} \downarrow_{S_{n-2,2}}\right) \geq 2 .
$$

Case 1. $\lambda_{1}-\lambda_{2} \equiv 0 \bmod p$. Note that $\lambda_{1} \equiv \lambda_{2} \equiv 0 \bmod p$. So

$$
D^{\lambda} \downarrow_{S_{n-2}} \cong D^{\left(\lambda_{1}-1, \lambda_{2}-1\right)} \oplus D^{\left(\lambda_{1}, \lambda_{2}-2\right)} \oplus e_{-2} D^{\left(\lambda_{1}-1, \lambda_{2}\right)},
$$

with $e_{-2} D^{\left(\lambda_{1}-1, \lambda_{2}\right)}$ indecomposable with simple head and socle and

$$
e_{-2} D^{\left(\lambda_{1}-1, \lambda_{2}\right)} \sim D^{\left(\lambda_{1}-1, \lambda_{2}-1\right)}|A| D^{\left(\lambda_{1}-1, \lambda_{2}-1\right)}
$$

with $\left[A: D^{\left(\lambda_{1}-1, \lambda_{2}-1\right)}\right]=0$. Further $D^{\left(\lambda_{1}-1, \lambda_{2}-1\right)} \otimes D^{\left(1^{2}\right)}$ is a composition factor of $D^{\lambda} \downarrow_{S_{n-2,2}}$ with multiplicity 1 by [11, Lemma 1.11]. So by self-duality of $D^{\lambda} \downarrow_{S_{n-2,2}}$ (or block decomposition) it follows that

$$
D^{\lambda} \downarrow_{\mathrm{S}_{n-2}} \cong\left(D^{\left(\lambda_{1}-1, \lambda_{2}-1\right)} \otimes D^{\left(1^{2}\right)}\right) \oplus\left(D^{\left(\lambda_{1}, \lambda_{2}-2\right)} \otimes D^{\nu}\right) \oplus B,
$$

with $\nu \in\left\{(2),\left(1^{2}\right)\right\}$ and $B$ indecomposible with simple head and socle isomorphic to $D^{\left(\lambda_{1}-1, \lambda_{2}-1\right)} \otimes D^{(2)}$ and no other such composition factor. It then follows that

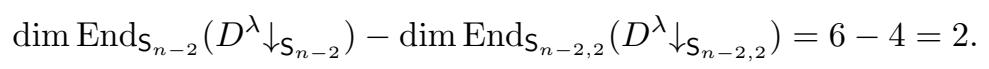

Case 2. $\lambda_{1}-\lambda_{2} \equiv-1 \bmod p$. In this case $\lambda_{1} \equiv(p-1) / 2 \bmod p$ and $\lambda_{2} \equiv(p+1) / 2$ $\bmod p$. So both removable nodes have the same residue. Then by [37, Lemma 4.2] we have that

$$
D^{\lambda} \downarrow_{\mathrm{S}_{n-2,2}} \cong\left(D^{\left(\lambda_{1}-1, \lambda_{2}-1\right)} \otimes D^{(2)}\right) \oplus\left(D^{\left(\lambda_{1}-1, \lambda_{2}-1\right)} \otimes D^{\left(1^{2}\right)}\right) \oplus B
$$

for a certain module $B$ and then

$$
D^{\lambda} \downarrow_{S_{n-2}} \cong\left(D^{\left(\lambda_{1}-1, \lambda_{2}-1\right)}\right)^{\oplus 2} \oplus B^{\prime}
$$

with $B^{\prime} \cong B \downarrow_{\mathrm{S}_{n-2}}$. It then follows that

$$
\operatorname{dim} \operatorname{End}_{S_{n-2}}\left(D^{\lambda} \downarrow_{S_{n-2}}\right)-\operatorname{dim} \operatorname{End}_{S_{n-2,2}}\left(D^{\lambda} \downarrow_{S_{n-2,2}}\right) \geq 2 .
$$

Lemma 5.5. Let $p \geq 5, n \geq 8, \lambda=\left(\lambda_{1}, \lambda_{2}\right) \in \mathscr{P}_{p}(n)$ with $\lambda_{2} \geq 2$. If $\lambda$ is JS then

$$
\operatorname{dim} \operatorname{Hom}_{\mathrm{S}_{n}}\left(\widetilde{D}_{k}, \operatorname{End}_{F}\left(D^{\lambda}\right)\right)= \begin{cases}1, & k \in\{0,3\} \\ 0, & \text { else. }\end{cases}
$$

Proof. We will use Lemma 2.2 throughout the proof without further reference to it.

If $h(\mu) \geq 5$ then $D^{\mu} \nsubseteq \operatorname{End}_{F}\left(D^{\lambda}\right)$, since $\lambda$ has only 2 rows (note that any composition factor of $D^{\lambda}$ is also a composition factor of $S^{\lambda} \otimes M^{\lambda} \cong S^{\lambda} \downarrow_{\mathrm{S}_{\lambda_{1}, \lambda_{2}}} \uparrow^{\mathrm{S}_{n}}$ ).

Since $\lambda \neq \lambda^{\mathrm{M}}$ by [33, Lemma 1.8], we have that $D^{(n)^{\mathrm{M}}} \nsubseteq \operatorname{End}_{F}\left(D^{\lambda}\right)$. So we only need to check the lemma for $k \leq 3$. For $k=0$ the lemma clearly holds.

If $\lambda_{1}>\lambda_{2}$ then $\lambda_{1} \geq \lambda_{2}+3$ since $\lambda$ is JS. If $\lambda_{1}=\lambda_{2}$ then $\lambda_{2} \geq 4$ since $n \geq 8$.

It can be checked that $D^{(2)}, D^{\left(1^{2}\right)}, D^{(2,1)}, D^{(3,1)}$ and $D^{\left(2^{2}\right)}$ are composition factors of $D^{\lambda} \downarrow_{s_{k}}$ for the corresponding $k$. Comparing dimensions and multiplicities 
as well as $D^{\lambda} \downarrow_{\mathrm{S}_{n-k, k}} \downarrow_{\mathrm{S}_{n-k-1,1, k}}$ and $D^{\lambda} \downarrow_{\mathrm{S}_{n-k-1, k+1}} \downarrow_{\mathrm{S}_{n-k-1,1, k}}$ we have that if $\lambda_{1}>\lambda_{2}$ then

$$
\begin{aligned}
D^{\lambda} \downarrow_{\mathrm{S}_{n-1}} \cong & D^{\left(\lambda_{1}-1, \lambda_{2}\right)}, \\
D^{\lambda} \downarrow_{\mathrm{S}_{n-2,2}} \cong & \left(D^{\left(\lambda_{1}-2, \lambda_{2}\right)} \otimes D^{(2)}\right) \oplus\left(D^{\left(\lambda_{1}-1, \lambda_{2}-1\right)} \otimes D^{\left(1^{2}\right)}\right), \\
D^{\lambda} \downarrow_{\mathrm{S}_{n-3,3}} \cong & \left(D^{\left(\lambda_{1}-3, \lambda_{2}\right)} \otimes D^{(3)}\right) \oplus\left(D^{\left(\lambda_{1}-2, \lambda_{2}-1\right)} \otimes D^{(2,1)}\right), \\
D^{\lambda} \downarrow_{\mathrm{S}_{n-4,4}} \cong & \left(D^{\left(\lambda_{1}-4, \lambda_{2}\right)} \otimes D^{(4)}\right)^{\oplus \delta_{\lambda_{1} \geq \lambda_{2}+4}} \oplus\left(D^{\left(\lambda_{1}-3, \lambda_{2}-1\right)} \otimes D^{(3,1)}\right) \\
& \oplus\left(D^{\left(\lambda_{1}-2, \lambda_{2}-2\right)} \otimes D^{\left(2^{2}\right)}\right),
\end{aligned}
$$

while $\lambda_{1}=\lambda_{2}$ and

$$
\begin{aligned}
D^{\lambda} \downarrow_{S_{n-1}} \cong & D^{\left(\lambda_{1}, \lambda_{2}-1\right)}, \\
D^{\lambda} \downarrow_{S_{n-2,2}} \cong & \left(D^{\left(\lambda_{1}, \lambda_{2}-2\right)} \otimes D^{(2)}\right) \oplus\left(D^{\left(\lambda_{1}-1, \lambda_{2}-1\right)} \otimes D^{\left(1^{2}\right)}\right), \\
D^{\lambda} \downarrow_{S_{n-3,3}} \cong & \left(D^{\left(\lambda_{1}, \lambda_{2}-3\right)} \otimes D^{(3)}\right) \oplus\left(D^{\left(\lambda_{1}-1, \lambda_{2}-2\right)} \otimes D^{(2,1)}\right), \\
D^{\lambda} \downarrow_{S_{n-4,4}} \cong & \left(D^{\left(\lambda_{1}, \lambda_{2}-4\right)} \otimes D^{(4)}\right)^{\oplus \delta_{p=5}} \oplus\left(D^{\left(\lambda_{1}-1, \lambda_{2}-3\right)} \otimes D^{(3,1)}\right) \\
& \oplus\left(D^{\left(\lambda_{1}-2, \lambda_{2}-2\right)} \otimes D^{\left(2^{2}\right)}\right)
\end{aligned}
$$

(in the first case as well as some parts in the second case this also follows from [11, Lemma 1.11] and by comparing multiplicities and dimensions).

From Lemma 3.11 we have that $\bar{D}_{2}$ or $\bar{D}_{3}$ is contained in $\operatorname{End}_{F}\left(D^{\lambda}\right)$. The lemma then follows from Lemma 4.4 or 4.5 together with Lemma 2.20.

Lemma 5.6. Let $p=3, n \geq 6$ with $n \equiv 0 \bmod 3$ and $\lambda=\left(\lambda_{1}, \lambda_{2}, \lambda_{3}\right) \in \mathscr{P}_{3}(n)$ with $\lambda_{1}>\lambda_{2}>\lambda_{3} \geq 1$. If $\lambda$ is not $J S$ then $\bar{D}_{0} \oplus \bar{D}_{1} \oplus \bar{D}_{2} \subseteq \operatorname{End}_{F}\left(D^{\lambda}\right)$ or $\bar{D}_{0} \oplus \bar{D}_{1} \oplus \bar{D}_{3} \subseteq \operatorname{End}_{F}\left(D^{\lambda}\right)$.

Proof. In view of Lemmas 2.23 and 3.11 we have that $\bar{D}_{2}$ or $\bar{D}_{3}$ is contained in $\operatorname{End}_{F}\left(D^{\lambda}\right)$. Thus it is enough to prove that $\bar{D}_{1} \cong D_{1} \subseteq \operatorname{End}_{F}\left(D^{\lambda}\right)$. By assumption $\lambda_{1}-\lambda_{2} \equiv \lambda_{2}-\lambda_{3} \bmod 3$ and we may assume that $\lambda_{1}-\lambda_{2} \not \equiv 1 \bmod 3$.

If $\lambda_{1}-\lambda_{2} \equiv 2 \bmod 3$ then $\lambda$ has 3 normal nodes. So $\operatorname{dim} \operatorname{End}_{\mathrm{s}_{n-3}}\left(D^{\lambda}\right)=3$ by Lemma 2.2. It then follows from Lemmas 2.20 and 4.5 that $D_{1} \subseteq \operatorname{End}_{F}\left(D^{\lambda}\right)$.

So assume now that $\lambda_{1}-\lambda_{2} \equiv 0 \bmod 3$. In this case if $i$ is the residue of $\left(1, \lambda_{1}\right)$ then $\varepsilon_{i}(\lambda)=1, \varepsilon_{i-1}(\lambda)=1, \varphi_{i}(\lambda) \geq 1$ and $\varphi_{i-1}(\lambda) \geq 1$. So, by Lemmas 2.2 and 2.3 ,

$$
D^{\lambda} \otimes M_{1} \cong f_{i} e_{i} D^{\lambda} \oplus f_{i-1} e_{i-1} D^{\lambda} \oplus M \sim\left(D^{\lambda}|\ldots| D^{\lambda}\right) \oplus\left(D^{\lambda}|\ldots| D^{\lambda}\right) \oplus M
$$

for a certain module $M$. It then follows from Lemma 4.5 that also in this case $D_{1} \subseteq \operatorname{End}_{F}\left(D^{\lambda}\right)$.

5.2. Spin representations. The results obtained in this section will only be used to obtain reduction to tensor products with the natural module and with the basic spin module for $p=3$. However we prove them in general, since the proof in the general case is not more complicated or much longer. We refer the reader to [12, §2-b] for the definition of even and odd homomorphisms.

Lemma 5.7. Let $A$ be a superalgebra and $M$ be an $A$-supermodule with $\mathrm{hd}(M) \cong D$ simple of type $Q$. If $\operatorname{End}_{A}(M) \simeq \operatorname{End}_{A}(D)^{[M: D]}$ then $M$ admits an odd involution. 
Proof. Note that $\operatorname{Hom}_{A}(M, \operatorname{rad} M) \simeq \operatorname{End}_{A}(D)^{[M: D]-1}$, since hd(M) $\cong D$ and $\operatorname{End}_{A}(M) \simeq \operatorname{End}_{A}(D)^{[M: D]}$. Since $D$ is of type Q, there are $[M: D]$ even and $[M: D]$ odd linearly independent homomorphisms in $\operatorname{End}_{A}(M)$ but only $[M: D]-1$ even and $[M: D]-1$ odd linearly independent homomorphisms in $\operatorname{Hom}_{A}(M, \operatorname{rad} M)$. The lemma then follows.

Lemma 5.8. Let $A$ and $B$ be superalgebras, $M$ be an $A$-supermodule and $N$ be a B-supermodule. If both $M$ and $N$ admit an odd involution then there exists an $A \otimes B$-supermodule $L$ such that $M \otimes N \cong L^{\oplus 2}$.

Proof. As in [12, §2-b] (in order for the argument to work it is not required that $M$ and $N$ are simple).

Lemma 5.9. Let $\nu \in \mathscr{R} \mathscr{P}_{p}(n-1)$. If $\varepsilon_{i}(\nu)>0$ then the following happen.

(i) If $D\left(\widetilde{e}_{i} \nu\right)$ is of type $M$ then $e_{i} D(\nu) \uparrow^{\widetilde{\varsigma}_{n-2,2}} \cong e_{i} D(\nu) \otimes D((2))=: e_{i} D(\nu) \circledast D((2))$.

(ii) If $D\left(\widetilde{e}_{i} \nu\right)$ is of type $Q$ then $e_{i} D(\nu) \uparrow^{\widetilde{S}_{n-2,2}} \cong e_{i} D(\nu) \otimes D((2)) \cong\left(e_{i} D(\nu) \circledast D((2))\right)^{\oplus 2}$ for a certain module $e_{i} D(\nu) \circledast D((2))$.

Further $e_{i} D(\nu) \circledast D((2))$ has simple head and socle isomorphic to $D\left(\widetilde{e}_{i} \nu,(2)\right)$ and $\operatorname{dim} \operatorname{End}_{\widetilde{\mathrm{S}}_{n-2,2}}\left(e_{i} D(\nu) \circledast D((2))\right)=\varepsilon_{i}(\nu) \operatorname{dim} \operatorname{End}\left(D\left(\widetilde{e}_{i} \nu,(2)\right)\right)$.

Proof. (i) clearly holds. For (ii) note that by Lemma 2.7. $\operatorname{hd}\left(e_{i} D(\nu)\right) \cong D\left(\tilde{e}_{i} \nu\right)$ and $\operatorname{End}_{\mathrm{S}_{n-2}}\left(e_{i} D(\nu)\right) \simeq \operatorname{End}_{\mathrm{S}_{n-2}}\left(D\left(\tilde{e}_{i} \nu\right)\right)^{\oplus\left[e_{i} D(\nu): D\left(\tilde{e}_{i} \nu\right)\right]}$. So $e_{i} D(\nu)$ admits an odd involution by Lemma 5.7 and then (ii) follows from Lemma 5.8

Further for any $\mu \in \mathscr{R} \mathscr{P}_{p}(n-2)$

$$
\begin{aligned}
& \operatorname{dim} \operatorname{Hom}_{\widetilde{\mathrm{S}}_{n-2,2}}\left(e_{i} D(\nu) \uparrow \widetilde{\mathrm{S}}_{n-2,2}, D(\mu,(2))\right) \\
& =\operatorname{dim} \operatorname{Hom}_{\widetilde{\mathrm{S}}_{n-2}}\left(e_{i} D(\nu), D(\mu,(2)) \downarrow_{\widetilde{\mathrm{S}}_{n-2}}\right) \\
& =2^{1-a(\mu)} \operatorname{dim} \operatorname{Hom}_{\widetilde{\mathrm{S}}_{n-2}}\left(e_{i} D(\nu), D(\mu)\right) \\
& =2 \delta_{\mu, \widetilde{e}_{i} \nu} .
\end{aligned}
$$

Since $D\left(\widetilde{e}_{i} \nu\right)$ and $D\left(\widetilde{e}_{i} \nu,(2)\right)$ are of different type, it follows that head and socle of $e_{i} D(\nu) \circledast D((2))$ are isomorphic to $D\left(\widetilde{e}_{i} \nu,(2)\right)$.

Last, from Lemma 2.7, we have that

$$
\begin{aligned}
& \operatorname{dim} \operatorname{End}_{\widetilde{\mathrm{S}}_{n-2,2}}\left(e_{i} D(\nu) \circledast D((2))\right) \\
& =2^{-2 a\left(\widetilde{e}_{i} \nu\right)} \operatorname{dim} \operatorname{End}_{\widetilde{\mathrm{S}}_{n-2,2}}\left(e_{i} D(\nu) \uparrow^{\widetilde{S}_{n-2,2}}\right) \\
& =2^{-2 a\left(\widetilde{e}_{i} \nu\right)} \operatorname{dim} \operatorname{Hom}_{\widetilde{\mathrm{S}}_{n-2}}\left(e_{i} D(\nu), e_{i} D(\nu) \uparrow^{\widetilde{S}_{n-2,2}} \downarrow_{\widetilde{\mathrm{S}}_{n-2}}\right) \\
& =2^{1-2 a\left(\widetilde{e}_{i} \nu\right)} \operatorname{dim} \operatorname{End}_{\widetilde{\mathrm{S}}_{n-2}}\left(e_{i} D(\nu)\right) \\
& =2^{1-a\left(\widetilde{e}_{i} \nu\right)} \varepsilon_{i}(\nu) \\
& =\varepsilon_{i}(\nu) \operatorname{dim} \operatorname{End}\left(D\left(\widetilde{e}_{i} \nu,(2)\right)\right)
\end{aligned}
$$

Lemma 5.10. Let $n \geq 5$ and $\lambda \in \mathscr{R} \mathscr{P}_{p}(n)$. If $\varepsilon_{0}(\lambda), \varepsilon_{i}(\lambda)=1$ and $\varepsilon_{j}(\lambda)=0$ for $j \neq 0, i$ then at least one of $\widetilde{e}_{0} \lambda$ and $\widetilde{e}_{j} \lambda$ is not JS. 
Proof. Notice first that $h(\lambda) \geq 2$. Assume that $\widetilde{e}_{j} \lambda$ is JS. Then it is JS(0) by Lemma 2.16. Since $\varphi_{j}\left(\widetilde{e}_{j} \lambda\right) \geq 1$, it follows from Lemma 2.13 that the top addable node of $\lambda$ is the only conormal node of $\widetilde{e}_{j} \lambda$ and this node has residue $j$. So the normal nodes of $\lambda$ are on row 1 (of residue $j$ ) and on row $h(\lambda)$ (of residue 0 ). It is easy to see that $\left(1, \lambda_{1}\right)$ is normal also in $\widetilde{e}_{0} \lambda$ (any removable node in $\lambda$ is also removable in $\widetilde{e}_{0} \lambda$ apart for the node $(h(\lambda), 1)$ and any addable node in $\widetilde{e}_{0} \lambda$ is also addable in $\lambda$ again apart the node $(h(\lambda), 1))$. Since $\widetilde{e}_{0} \lambda$ is JS it follows that $\lambda=(n-1,1)$. From $\widetilde{e}_{j} \lambda=(n-2,1)$ being $\operatorname{JS}(0)$ it follows from [43, Lemma 3.7] that $\lambda=(3,1)$ or $(p, 1)$. The first case contradicts $n \geq 5$ while in the second case both removable nodes have residue 0 .

Lemma 5.11. Let $\lambda \in \mathscr{R} \mathscr{P}_{p}(n)$ be $\lambda \in J S(0)$. Then $\varphi_{0}(\lambda) \geq 1$ if and only if $n \equiv 0 \bmod p$.

Proof. Assume first that $\lambda \in \mathrm{JS}(0)$ and $n \equiv 0 \bmod p$. From Lemma 4.2 we have that $\left[D(\lambda) \otimes M^{(n-1,1)}: D(\lambda)\right] \geq 2$. So from Lemma 2.12 it follows that $\varphi_{0}(\lambda) \geq 1$.

Assume now that $\lambda \in J S(0)$ and $\varphi_{0}(\lambda) \geq 1$. Then all normal and conormal nodes of $\lambda$ have residue 0 by Lemma 2.13. In particular the top addable node has residue 0 . So $\lambda_{1} \equiv 0$ or $-1 \bmod p$.

If $\lambda_{1} \equiv-1 \bmod p$ then from [43, Lemma 3.7] we have that $\bar{\lambda}:=\left(\lambda_{1}+1, \lambda_{1}, \lambda_{2}, \ldots\right)$ $\in J S(0)$. Since $|\lambda| \equiv|\bar{\lambda}| \bmod p$, we may assume that $\lambda_{1} \equiv 0 \bmod p$.

For a residue $i$ define

$$
\begin{aligned}
& A_{i}:=\left\{2 \leq j \leq h(\lambda) \mid \operatorname{res}\left(j, \lambda_{j}\right)=i=\operatorname{res}\left(j-1, \lambda_{j-1}\right)-1\right\}, \\
& B_{i}:=\left\{2 \leq j \leq h(\lambda) \mid \operatorname{res}\left(j, \lambda_{j}\right)=i=\operatorname{res}\left(j-1, \lambda_{j-1}\right)+1\right\}, \\
& C_{i}:=\left\{2 \leq j \leq h(\lambda) \mid \operatorname{res}\left(j, \lambda_{j}\right)=i=\operatorname{res}\left(j-1, \lambda_{j-1}\right)\right\} .
\end{aligned}
$$

From [43, Lemma 3.7] we have the following:

- $\cup_{i}\left(A_{i} \cup B_{i} \cup C_{i}\right)=\{2, \ldots, h(\lambda)\}$,

- if $C_{i} \neq \emptyset$ then $i=0$,

- if $j \in C_{0}$ then $\lambda_{j} \equiv 0 \bmod p$,

- if $j \in A_{i}$ then $\lambda_{j} \equiv i+1 \bmod p$,

- if $j \in B_{i+1}$ then $\lambda_{j} \equiv-i-1 \bmod p$.

Since $\operatorname{res}\left(1, \lambda_{1}\right)=0=\operatorname{res}\left(h(\lambda), \lambda_{h(\lambda)}\right)$ it then follows that $\left|A_{i}\right|=\left|B_{i+1}\right|$ for each $0 \leq i<(p-1) / 2$. In particular

$$
\begin{aligned}
|\lambda| & =\lambda_{1}+\sum_{i=0}^{(p-3) / 2} \sum_{j \in A_{i}} \lambda_{j}+\sum_{i=1}^{(p-1) / 2} \sum_{j \in B_{i}} \lambda_{j}+\sum_{j \in C_{0}} \lambda_{j} \\
& \equiv \sum_{i=0}^{(p-3) / 2}\left(\left|A_{i}\right|(i+1)-\left|B_{i+1}\right|(i+1)\right) \equiv 0 \bmod p .
\end{aligned}
$$

Remark 5.12. Let $\lambda \in \mathscr{R} \mathscr{P}_{p}(n)$ be $\operatorname{JS}(0)$ with $\varphi_{0}(\lambda) \geq 1$. Then by Lemma 2.13 we have that $\varphi_{0}(\lambda)=2$ and $\varphi_{i}(\lambda)=0$ for $i>0$. From Lemmas 2.6] and 2.16] we have that $\widetilde{f}_{0} \lambda$ only has normal nodes of residue 0 . So it can be seen that the following are equivalent:

- $\lambda$ is $\operatorname{JS}(0)$ with $\varphi_{0}(\lambda) \geq 1$,

- $\lambda=\widetilde{e}_{0} \mu$ is $\operatorname{JS}(0)$ and all normal nodes of $\mu$ have residue 0 . 
This holds for example if $p=5$ and $\lambda=(4,3,2,1)=\widetilde{e}_{0}(5,3,2,1)$. Note that $(5,3,2,1) \neq\left(5^{2}, 1\right)=\beta_{11}$. This shows that [43, Lemma 3.14(i)] is wrong. Since it is unclear where the error is in the proof of [43, Lemma 3.14] we next give a different proof of [43, Lemma 3.14(ii)].

Lemma 5.13. Let $\lambda \in \mathscr{R} \mathscr{P}_{p}(n)$. Then $\lambda \in J S(i)$ and $\widetilde{e}_{i} \lambda \in J S(j)$ for some $i, j \neq 0$ if and only if $\lambda=\beta_{n}$ with $n \not \equiv 0,1,2 \bmod p$.

Proof. For $\lambda=\beta_{n}$ it can be easily checked that $\lambda \in J S(i)$ and $\widetilde{e}_{i} \lambda \in J S(j)$ for some $i, j \neq 0$ if and only if $n \not \equiv 0,1,2 \bmod p$.

So assume that $\lambda \in J S(i)$ and $\widetilde{e}_{i} \lambda \in J S(j)$ for some $i, j \neq 0$. Notice that the only normal node of $\lambda$ ( of $\widetilde{e}_{i} \lambda$ ) is the last node on the bottom row, since $\lambda\left(\widetilde{e}_{i} \lambda\right.$ ) is JS. It then easily follows from $i, j \neq 0$ that $h(\lambda)=h\left(\widetilde{e}_{i} \lambda\right)$, that $3 \leq \lambda_{h(\lambda)}<p$ and $\widetilde{e}_{i} \lambda=$ $\left(\lambda_{1}, \ldots, \lambda_{h(\lambda)-1}, \lambda_{h(\lambda)}-1\right)$. If $p \mid \lambda_{k}$ for each $1 \leq k<h(\lambda)$ then $\lambda=\left(p^{h(\lambda)-1}, \lambda_{h(\lambda)}\right)$ and so $\lambda=\beta_{n}$ and $n \not \equiv 0,1,2 \bmod p$. So assume that this is not the case and let $k<h(\lambda)$ maximal such that $p \nmid \lambda_{k}$. Notice that $\lambda=\left(\lambda_{1}, \ldots, \lambda_{k}, p^{h(\lambda)-k-1}, \lambda_{h(\lambda)}\right)$. Since $\lambda$ is JS it can be checked that $\operatorname{res}\left(k, \lambda_{k}\right)=\operatorname{res}\left(h(\lambda), \lambda_{h(\lambda)}+1\right)$. On the other hand, since $\widetilde{e}_{i} \lambda=\left(\lambda_{1}, \ldots, \lambda_{k}, p^{h(\lambda)-k-1}, \lambda_{h(\lambda)}-1\right)$ is also JS, we have that $\operatorname{res}\left(k, \lambda_{k}\right)=\operatorname{res}\left(h(\lambda), \lambda_{h(\lambda)}\right)$. In particular $\operatorname{res}\left(h(\lambda), \lambda_{h(\lambda)}\right)=\operatorname{res}\left(h(\lambda), \lambda_{h(\lambda)}+1\right)$ and so $p \mid \lambda_{h(\lambda)}$, contradicting $\lambda \in \mathscr{R} \mathscr{P}_{p}(n)$.

Lemma 5.14. Let $n \geq 5$ and $\lambda \in \mathscr{R} \mathscr{P}_{p}(n) \backslash\left\{\beta_{n}\right\}$. Then

$$
\operatorname{dim} \operatorname{End}_{\widetilde{\mathbf{S}}_{n-2,2}}\left(D(\lambda) \downarrow_{\widetilde{\mathbf{S}}_{n-2,2}}\right)>\operatorname{dim} \operatorname{End}_{\widetilde{\mathbf{S}}_{n-1}}\left(D(\lambda) \downarrow{\widetilde{\widetilde{S}_{n-1}}}\right)+\operatorname{dim} \operatorname{End}_{\widetilde{\boldsymbol{S}}_{n}}(D(\lambda))
$$

unless one of the following holds:

- $\lambda$ is JS(1), $p=3$ and $\varepsilon_{0}\left(\widetilde{e}_{1} \lambda\right)=3$,

- $\lambda$ is $J S(1), p>3, \varepsilon_{0}\left(\widetilde{e}_{1} \lambda\right)=1$ and $\varepsilon_{2}\left(\widetilde{e}_{1} \lambda\right)=1$,

- $\varepsilon_{0}(\lambda)=2, \varepsilon_{i}(\lambda)=0$ for $i>0$ and $\widetilde{e}_{0} \lambda \in \operatorname{JS}(0)$,

$-\lambda$ is $J S(0)$.

Proof. Throughout the proof let $\varepsilon_{i}:=\varepsilon_{i}(\lambda)$ and for $\alpha \in \mathscr{P}(n)$ let

$$
d_{\alpha}:=\operatorname{dim} \operatorname{End}_{\widetilde{\mathbf{S}}_{\alpha}}\left(D(\lambda) \downarrow_{\widetilde{\mathrm{S}}_{\alpha}}\right) .
$$

We will use Lemma 2.7 without further referring to it.

Note that

$$
D(\lambda) \downarrow_{\widetilde{\mathrm{S}}_{n-2,2}} \cong \bigoplus_{i} E_{i} \oplus \bigoplus_{i<j} E_{i, j},
$$

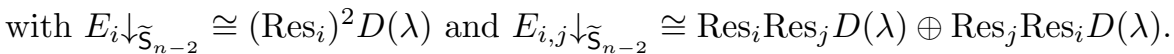

Further, since $M^{(n-2)} \otimes M^{\left(1^{2}\right)} \cong(\mathbf{1} \bigotimes \mathbf{1}) \oplus(\mathbf{1} \bigotimes \operatorname{sgn})$, we have that $E_{i \downarrow s_{n-2}} \uparrow^{\widetilde{S}_{n-2,2}} \cong$ $E_{i} \oplus E_{i}^{\prime}$ with $E_{i}^{\prime} \cong E_{i} \otimes(\mathbf{1} \otimes \operatorname{sgn})$ and $E_{i, j} \downarrow_{\widetilde{S}_{n-2}} \uparrow^{\widetilde{S}_{n-2,2}} \cong E_{i, j} \oplus E_{i, j}^{\prime}$ with $E_{i, j}^{\prime} \cong$ $E_{i, j} \otimes(1 \otimes \operatorname{sgn})$. In particular

$$
\operatorname{dim} \operatorname{End}_{\widetilde{\mathbf{S}}_{n-2,2}}\left(E_{i}\right)=\operatorname{dim} \operatorname{End}_{\widetilde{\mathbf{S}}_{n-2,2}}\left(E_{i}^{\prime}\right)
$$

and

$$
\operatorname{dim} \operatorname{End}_{\widetilde{\mathrm{S}}_{n-2,2}}\left(E_{i, j}\right)=\operatorname{dim} \operatorname{End}_{\widetilde{\mathrm{S}}_{n-2,2}}\left(E_{i, j}^{\prime}\right) .
$$

Consider first $E_{i}$. If $\varepsilon_{i}>0$ then

$$
\left(e_{i} D\left(\widetilde{e}_{i} \lambda\right)\right)^{\oplus 2+2 \delta_{i>0}} \subseteq\left(e_{i}^{(2)} D(\lambda)\right)^{\oplus 2+2 \delta_{i>0}} \subseteq E_{i \downarrow}{\widetilde{\varsigma-2}}_{n} .
$$


In particular $A=\left(e_{i} D\left(\widetilde{e}_{i} \lambda\right) \circledast D((2))\right)^{\oplus\left(2+2 \delta_{i>0}\right)(1+a(\lambda))} \subseteq E_{i} \oplus E_{i}^{\prime}$. So

$$
\left(e_{i} D\left(\widetilde{e}_{i} \lambda\right) \circledast D((2))\right)^{\oplus\left(1+\delta_{i}>0\right)\left(1+a\left(\widetilde{e}_{i} \lambda\right)\right)}
$$

is contained in $E_{i}$ or $E_{i}^{\prime}$ from Lemma 5.9 and similarly to [37, Lemma 3.7]. Due to self-duality of the modules it then follows that

$$
\operatorname{dim} \operatorname{End}_{\widetilde{\mathrm{S}}_{n-2,2}}\left(E_{i}\right) \geq 2 \delta_{\varepsilon_{i}>0}\left(1+\delta_{i>0}\right)^{2}\left(\varepsilon_{i}-1\right) d_{(n)} .
$$

Consider next $E_{i, j}$ with $0<i<j$. Assume that $\varepsilon_{i}, \varepsilon_{j}>0$. Then

$$
\left(e_{i} D\left(\widetilde{e}_{j} \lambda\right) \oplus e_{j} D\left(\widetilde{e}_{i} \lambda\right)\right)^{\oplus 2} \subseteq E_{i, j} \downarrow_{\widetilde{s}_{n-2}} .
$$

In particular

$$
\left(e_{i} D\left(\widetilde{e}_{j} \lambda\right) \circledast D((2)) \oplus e_{j} D\left(\widetilde{e}_{i} \lambda\right) \circledast D((2))\right)^{\oplus 2+2 a(\lambda)} \subseteq E_{i, j} \oplus E_{i, j}^{\prime} .
$$

Let $\{k, l\}=\{i, j\}$ with $\varepsilon_{k}\left(\widetilde{e}_{l} \lambda\right) \geq \varepsilon_{l}\left(\widetilde{e}_{k} \lambda\right)$. Then one of $\left(e_{i} D\left(\widetilde{e}_{j} \lambda\right) \circledast D((2)) \oplus e_{j} D\left(\widetilde{e}_{i} \lambda\right) \circledast D((2))\right)^{\oplus 1+a(\lambda)} \quad$ or $\quad\left(e_{k} D\left(\widetilde{e}_{l} \lambda\right) \circledast D((2))\right)^{\oplus 2+a(\lambda)}$ is contained in $E_{i, j}$ or $E_{i, j}^{\prime}$. In either case it follows from $\varepsilon_{a}\left(\widetilde{e}_{b} \lambda\right) \geq \varepsilon_{a}$ (see Lemma 2.16) and from Lemma 2.15 that

$$
\begin{aligned}
\operatorname{dim} \operatorname{End}_{\widetilde{\mathrm{S}}_{n-2,2}}\left(E_{i, j}\right) & >2 \delta_{\varepsilon_{i}>0} \delta_{\varepsilon_{j}>0}\left(\varepsilon_{i}\left(\widetilde{e}_{j} \lambda\right)+\varepsilon_{j}\left(\widetilde{e}_{i} \lambda\right)\right) d_{(n)} \\
& \geq 2 \delta_{\varepsilon_{i}>0} \delta_{\varepsilon_{j}>0}\left(\varepsilon_{i}+\varepsilon_{j}\right) d_{(n)} .
\end{aligned}
$$

Last consider $E_{0, i}$ with $i>0$. Again assume that $\varepsilon_{0}, \varepsilon_{i}>0$. Then

$$
\left(e_{0} D\left(\widetilde{e}_{i} \lambda\right) \oplus e_{i} D\left(\widetilde{e}_{0} \lambda\right)\right)^{\oplus 1+a(\lambda)} \subseteq E_{0, i} \downarrow_{\widetilde{\mathrm{S}}_{n-2}} .
$$

In particular

$$
\left(e_{0} D\left(\widetilde{e}_{i} \lambda\right) \circledast D((2)) \oplus e_{i} D\left(\widetilde{e}_{0} \lambda\right) \circledast D((2))\right)^{\oplus 2} \subseteq E_{i, j} \oplus E_{i, j}^{\prime} .
$$

Similar to the previous case we obtain

$$
\begin{aligned}
\operatorname{dim} \operatorname{End}_{\widetilde{\mathrm{S}}_{n-2,2}}\left(E_{0, i}\right) & >\delta_{\varepsilon_{0}>0} \delta_{\varepsilon_{i}>0}\left(\varepsilon_{0}\left(\widetilde{e}_{i} \lambda\right)+\varepsilon_{i}\left(\widetilde{e}_{0}(\lambda)\right) d_{(n)}\right. \\
& \geq \delta_{\varepsilon_{0}>0} \delta_{\varepsilon_{i}>0}\left(\varepsilon_{0}+\varepsilon_{i}\right) d_{(n)} .
\end{aligned}
$$

In particular, if $x=\left|\left\{j>0: \varepsilon_{j}>0\right\}\right|$,

$$
d_{(n-2,2)} \geq\left(\delta_{\varepsilon_{0}>0}\left((2+x) \varepsilon_{0}-2\right)+\sum_{i>0} \delta_{\varepsilon_{i}>0}\left(\left(6+2 x+\delta_{\varepsilon_{0}>0}\right) \varepsilon_{i}-8\right)\right) d_{(n)} .
$$

In view of Lemma 2.11 we may assume that

$$
d_{(n-2,2)} \leq d_{(n-1,1)}+d_{(n)}=\left(1+\varepsilon_{0}+2 \sum_{i>0} \varepsilon_{i}\right) d_{(n)} .
$$

It easily follows that $x+\delta_{\varepsilon_{0}>0} \leq 2$ and that we are in one of the following cases:

- $\varepsilon_{0} \leq 3$ and $\varepsilon_{k}=0$ for $k>0$.

- $\varepsilon_{0} \leq 2, \varepsilon_{i}=1$ and $\varepsilon_{k}=0$ for $k \neq 0, i$ for some $i>0$.

- $\varepsilon_{i}, \varepsilon_{j}=1$ and $\varepsilon_{k}=0$ for $k \neq i, j$ for some $i, j>0$.

Excluding cases which are not considered in the lemma and considering the stronger bounds involving $\varepsilon_{i}\left(\widetilde{e}_{j} \lambda\right)$, strict inequalities and that $E_{i, j} \neq 0$ if $\varepsilon_{i}>0$ and $\varepsilon_{j}\left(\widetilde{e}_{i} \lambda\right)>$ 0 , we may assume that we are in one of the following cases:

(a) $\varepsilon_{0}=3, \varepsilon_{k}=0$ and $\varepsilon_{k}\left(\widetilde{e}_{0} \lambda\right)>0$ for $k>0$,

(b) $\varepsilon_{0}=2, \varepsilon_{k}=0$ for $k>0$ and there exists $i>0$ with $\varepsilon_{i}\left(\widetilde{e}_{0} \lambda\right)>0$,

(c) $\lambda$ is $\operatorname{JS}(i)$ with $i>1$, 
(d) $p=3, \lambda$ is $\operatorname{JS}(1)$ and $\varepsilon_{0}\left(\widetilde{e}_{1} \lambda\right) \neq 3$,

(e) $p>3, \lambda$ is $\operatorname{JS}(1)$ and $\left(\varepsilon_{0}\left(\widetilde{e}_{1} \lambda\right), \varepsilon_{2}\left(\widetilde{e}_{1} \lambda\right)\right) \neq(1,1)$,

(f) $\varepsilon_{0}, \varepsilon_{i}=1, \varepsilon_{k}=0$ for $k \neq 0, i$ and $\varepsilon_{i}\left(\widetilde{e}_{0} \lambda\right)+\varepsilon_{0}\left(\widetilde{e}_{i} \lambda\right) \leq 3$ for some $i>0$,

(g) $\varepsilon_{i}, \varepsilon_{j}=1, \varepsilon_{k}=0$ for $k \neq i, j$ and $\widetilde{e}_{i} \lambda$ and $\widetilde{e}_{j} \lambda$ are JS for some $i, j>0$.

Case (a). In this case $D(\lambda) \downarrow_{\widetilde{s}_{n-2}} \cong e_{0}^{(2)} D(\lambda)^{\oplus 2}$ and

$$
\left[e_{0}^{(2)} D(\lambda): D\left(\widetilde{e}_{0}^{2} \lambda\right)\right]=3>2=\left[e_{0} D\left(\tilde{e}_{0} \lambda\right): D\left(\widetilde{e}_{0}^{2} \lambda\right)\right] .
$$

It can then be checked that $\left(e_{0} D\left(\widetilde{e}_{0} \lambda\right) \circledast D(2)\right)^{\oplus 1+a(\lambda)}$ is strictly contained in $E_{0}$ or $E_{0}^{\prime}$. Thus

$$
\begin{aligned}
\operatorname{dim} \operatorname{End}_{\widetilde{\mathrm{S}}_{n-2,2}}\left(D(\lambda) \downarrow_{\widetilde{\mathrm{S}}_{n-2,2}}\right) & >(1+a(\lambda))^{2}\left(\varepsilon_{0}-1\right) \operatorname{dim} \operatorname{End}_{\widetilde{\mathrm{S}}_{n-2,2}}\left(D\left(\widetilde{e}_{0}^{2} \lambda,(2)\right)\right) \\
= & 4 \operatorname{dim} \operatorname{End}_{\widetilde{\mathrm{S}}_{n}}(D(\lambda)) .
\end{aligned}
$$

So also in this case the lemma holds.

Case (b). In this case $\operatorname{dim} \operatorname{End}_{\widetilde{\mathrm{S}}_{n-2,2}}\left(E_{0}\right) \geq 2 \operatorname{dim} \operatorname{End}_{\widetilde{\mathrm{S}}_{n}}(D(\lambda))$, so it is enough to prove that $\operatorname{dim} \operatorname{End}_{\widetilde{\mathrm{S}}_{n-2,2}}\left(E_{0, i}\right)>\operatorname{dim} \operatorname{End}_{\widetilde{\mathrm{S}}_{n}}(D(\lambda))$ by Lemma 2.13. This follows from $E_{0, i}$ not being zero or simple as supermodule (since $\varepsilon_{0}(\lambda)=2$ and $\varepsilon_{i}\left(\widetilde{e}_{0} \lambda\right)>0$ ) and since its composition factors are of the same type as $D(\lambda)$.

Case (c). Using argument similar to the above we have (letting $E_{i, j}=E_{j, i}$ and $E_{i, j}^{\prime}=E_{j, i}^{\prime}$ for $\left.i>j\right)$ that $\left(e_{j} D\left(\widetilde{e}_{i} \lambda\right) \circledast D((2))\right)^{\oplus 1+a(\lambda)}$ is contained in $E_{i, j}$ or $E_{i, j}^{\prime}$ for each $j \neq i$ with $j>0$. From Lemma [5.13 and [43. Lemma 3.8] we have that $\sum_{j \neq i} \varepsilon_{j}\left(\widetilde{e}_{i} \lambda\right) \geq 2$. From [30, Lemma 20.2.3] we have that $\varepsilon_{0}\left(\widetilde{e}_{i} \lambda\right)=0$. The lemma then follows.

Case $(\mathrm{d})$. Notice that $e_{0} D\left(\widetilde{e}_{1} \lambda\right) \circledast D((2))$ is contained in $E_{0,1}$ or $E_{0,1}^{\prime}$. Since $\lambda \neq \beta_{n}$ it can be easily checked that $\lambda$ ends by $\left(4,3^{b}, 2\right)$ with $b \geq 0$. It can then be easily checked that $\varepsilon_{0}\left(\widetilde{e}_{1} \lambda\right) \geq 3$. So in this case $\varepsilon_{0}\left(\widetilde{e}_{1} \lambda\right) \geq 4$, from which the lemma follows.

Case (e). From [30, Lemma 20.2.3] and since $\lambda \in \operatorname{JS}(1)$ we have that $\varepsilon_{k}\left(\widetilde{e}_{1} \lambda\right)=0$ for $k \neq 0,2$. If $\lambda_{h(\lambda)}=p-1$ then the bottom removable node of $\widetilde{e}_{1} \lambda$ is 2 -normal (since $p>3$ ). If $\lambda_{h(\lambda)}=2$ let $k<h(\lambda)$ maximal with $p \nmid \lambda_{k}$. Note that $k$ exists since $\lambda \neq \beta_{n}$ From $\lambda \in \operatorname{JS}(1)$ it follows that $\operatorname{res}\left(k, \lambda_{k}\right)=2$ and by maximality of $k$ we have that $\left(k, \lambda_{k}\right)$ is normal for $\widetilde{e}_{1} \lambda$. In particular $\varepsilon_{2}\left(\widetilde{e}_{1} \lambda\right) \geq 1$.

We have that $\left(e_{0} D\left(\widetilde{e}_{1} \lambda\right) \circledast D((2))\right)^{\oplus 2}$ is contained in $E_{0,1}$ or $E_{0,1}^{\prime}$ and $\left(e_{j} D\left(\widetilde{e}_{i} \lambda\right) \circledast\right.$ $D((2)))^{\oplus 1+a(\lambda)}$ is contained in $E_{i, j}$ or $E_{i, j}^{\prime}$ for each $j \neq i$ with $j>0$. Since $\widetilde{e}_{1} \lambda$ is not JS by Lemma 5.13 and [43, Lemma 3.8], we have $\varepsilon_{2}\left(\widetilde{e}_{1} \lambda\right) \geq 2$ or $\varepsilon_{0}\left(\widetilde{e}_{1} \lambda\right), \varepsilon_{2}\left(\widetilde{e}_{1} \lambda\right) \geq 1$ from which the lemma follows.

Case (f). From Lemma 5.10 we have that $\widetilde{e}_{0}(\lambda)$ and $\widetilde{e}_{i}(\lambda)$ are not both JS. Since $\varepsilon_{i}\left(\widetilde{e}_{0} \lambda\right)+\varepsilon_{0}\left(\widetilde{e}_{i} \lambda\right) \leq 3$, we have by Lemmas 2.7 and 2.15 that $\widetilde{e}_{i} \widetilde{e}_{0} \lambda=\widetilde{e}_{0} \widetilde{e}_{i} \lambda$.

If $\varepsilon_{i}\left(\widetilde{e}_{0} \lambda\right)+\varepsilon_{0}\left(\widetilde{e}_{i} \lambda\right)=3$ the lemma follows from

$$
\left(e_{0} D\left(\widetilde{e}_{i} \lambda\right) \circledast D((2)) \oplus e_{i} D\left(\widetilde{e}_{0} \lambda\right) \circledast D((2))\right)
$$

being contained in $E_{0, i}$ or $E_{0, i}^{\prime}$ or $\left(e_{k} D\left(\widetilde{e}_{l} \lambda\right) \circledast D((2))\right)^{\oplus 2}$ being contained in one of $E_{0, i}$ or $E_{0, i}^{\prime}\left(\right.$ with $\{k, l\}=\{0, i\}$ such that $\left.\varepsilon_{k}\left(\widetilde{e}_{l} \lambda\right)=2\right)$.

If $\varepsilon_{i}\left(\widetilde{e}_{0} \lambda\right)+\varepsilon_{0}\left(\widetilde{e}_{i} \lambda\right)=2$ then $E_{0, i}$ is not the only non-zero block component of $D(\lambda) \downarrow_{\widetilde{S}_{n-2,2}}$, since $\widetilde{e}_{0}(\lambda)$ and $\widetilde{e}_{i}(\lambda)$ are not both JS. From $\widetilde{e}_{i} \widetilde{e}_{0} \lambda=\widetilde{e}_{0} \widetilde{e}_{i} \lambda$ we have that $\left(D\left(\widetilde{e}_{i} \widetilde{e}_{0} \lambda\right) \circledast D((2))\right)^{\oplus 2} \subseteq E_{0, i}$ or $E_{0, i}^{\prime}$, from which the lemma then follows. 
Case (g). In this case from Lemma 2.15 we have that $\widetilde{e}_{i} \widetilde{e}_{j} \lambda=\widetilde{e}_{j} \widetilde{e}_{i} \lambda$ and then $D\left(\widetilde{e}_{i} \widetilde{e}_{j} \lambda\right)^{\oplus 4}$ is contained in $D(\lambda) \downarrow_{\widetilde{S}_{n-2}}$. So $\left(D\left(\widetilde{e}_{i} \widetilde{e}_{j} \lambda\right) \circledast D((2))\right)^{\oplus 2+2 a(\lambda)}$ is contained in $E_{i, j}$ or $E_{i, j}^{\prime}$, from which the lemma follows by Lemma 2.13 ,

Lemma 5.15. Let $\lambda \in \mathscr{R} \mathscr{P}_{p}(n) \backslash\left\{\beta_{n}\right\}$ with $\varepsilon_{i}(\lambda)=0$ for all $i \neq 0$ and $\widetilde{e}_{0} \lambda \in \operatorname{JS}(0)$; then $D(\lambda) \downarrow_{S_{n-1}}$ has a composition factor $D(\mu)$, where $\mu \neq \widetilde{e}_{0} \lambda$ is obtained from $\lambda$ by removing the bottom removable node.

Proof. Let $A=\left(h, \lambda_{h}\right)$ be the bottom removable node of $\lambda$. Then $A$ is normal for $\lambda$. Since all normal nodes of $\lambda$ have residue 0 and $\widetilde{e}_{0} \lambda \in \operatorname{JS}(0)$, we have that $A$ is not good, so $\mu \neq \widetilde{e}_{0} \lambda$. By Lemma 2.9 it is then enough to prove that $\mu \in \mathscr{R} \mathscr{P}_{p}(n-1)$. Note that $A$ has residue 0 , so $\lambda_{h}=1$. If $\mu \notin \mathscr{R} \mathscr{P}_{p}(n-1)$ then $\lambda_{h-1}=p$. So the node $B:=(h-1, p)$ is also normal for $\lambda$. Since $\widetilde{e}_{0} \lambda \in \operatorname{JS}(0)$ we have that $\varepsilon_{0}(\lambda)=2$. In particular $B$ is the 0 -good node of $\lambda$. Let $k<h-1$ be maximal with $\lambda_{k}>p$ (such $k$ exists since $\lambda \neq \beta_{n}$ ). By [43, Lemma 3.7] it follows that $\lambda_{k}=p+1$. In particular the node $(k, p+1)$ is removable of residue 0 for $\lambda$, and then it is also 0 -normal, contradicting $B$ being the 0 -good node of $\lambda$.

Lemma 5.16. Let $\lambda \in \mathscr{R} \mathscr{P}_{p}(n)$ and $n \geq 4$. If $D(\lambda)$ is of type $Q$ then, in the Grothendieck group, $\left[D(\lambda,+) \downarrow_{\widetilde{\mathrm{S}}_{n-2}}\right]=\left[D(\lambda,-) \downarrow_{\widetilde{\mathrm{s}}_{n-2}}\right]$. If $D(\lambda)$ is of type $M$ then $\left[E(\lambda,+) \downarrow_{\widetilde{\mathrm{A}}_{n-2}}\right]=\left[E(\lambda,-) \downarrow_{\widetilde{\mathrm{A}}_{n-2}}\right]$.

Proof. Let $\mu \in \mathscr{R} \mathscr{P}_{p}(m)$ for some $m \geq 0$. It can be checked by definition of residues that

$$
\left.m-h_{p^{\prime}}(\mu) \equiv \mid \text { nodes of } \mu \text { of residue } \neq 0\right\} \mid \bmod 2 .
$$

So if $i=0$ then all composition factors of $\operatorname{Res}_{i} D(\mu)$ are of the same type as $D(\mu)$, while if $i>0$ then all composition factors of $\operatorname{Res}_{i} D(\mu)$ are of type different from the type of $D(\mu)$.

Assume first that $D(\lambda)$ is of type $\mathrm{Q}$. Then

$$
D(\lambda, \pm) \downarrow_{\widetilde{\mathrm{S}}_{n-2}} \cong \bigoplus_{i} D_{i, i}^{ \pm} \oplus \bigoplus_{i<j} D_{i, j}^{ \pm}
$$

with $D_{i, i}^{ \pm} \cong \operatorname{Res}_{i}^{2} D(\lambda, \pm)$ and $D_{i, j}^{ \pm} \cong \operatorname{Res}_{i} \operatorname{Res}_{j} D(\lambda, \pm) \oplus \operatorname{Res}_{j} \operatorname{Res}_{i} D(\lambda, \pm)$. Further $D(\lambda, \pm) \downarrow_{\widetilde{\mathrm{s}}_{n-2,2}} \cong \bigoplus_{i} E_{i, i}^{ \pm} \oplus \bigoplus_{i<j} E_{i, j}^{ \pm}$with $E_{i, j}^{ \pm} \downarrow_{\widetilde{s}_{n-2,2}} \cong D_{i, j}^{ \pm}$for $i \leq j$. For any $i \leq j$ we have $D_{i, j}^{+} \otimes \operatorname{sgn} \cong D_{i, j}^{-}$and $E_{i, j}^{+} \otimes \operatorname{sgn} \cong E_{i, j}^{-}$. If $j>0$ we then easily have that $\left[D_{0, j}^{+}\right]=\left[D_{0, j}^{-}\right]$, since composition factors of $D_{0, j}^{ \pm}$are of the form $D(\mu, 0) \cong$ $D(\mu, 0) \otimes \operatorname{sgn}$ for some $\mu \in \mathscr{R} \mathscr{P}_{p}(n-2)$. If $0<i \leq j$ then $\left[E_{i, j}^{+}\right]=\left[E_{i, j}^{-}\right]$, since composition factors of $E_{i, j}^{ \pm}$are of the form $D(\mu,(2)) \cong(D(\mu,(2))) \otimes \operatorname{sgn}$ for some $\mu \in \mathscr{R} \mathscr{P}_{p}(n-2)$. Also in this case it then follows that $\left[D_{i, j}^{+}\right]=\left[D_{i, j}^{-}\right]$.

If $D(\lambda)$ is of type M use a similar argument involving conjugation with $\widetilde{(1,2)}$ instead of tensoring with sgn.

Lemma 5.17. Let $n \geq 4, \lambda \in \mathscr{R} \mathscr{P}_{p}(n) \backslash\left\{\beta_{n}\right\}$. Let $G=\widetilde{\mathrm{S}}_{n}$ or $G=\widetilde{\mathrm{A}}_{n}$ and $D$ be a simple FG-module indexed by $\lambda$. Assume that one of the following holds:

(i) $\lambda$ is JS(1), $p=3$ and $\varepsilon_{0}\left(\widetilde{e}_{1} \lambda\right)=3$,

(ii) $\lambda$ is $J S(1), p>3$ and $\varepsilon_{0}\left(\widetilde{e}_{1} \lambda\right)=1$ and $\varepsilon_{2}\left(\widetilde{e}_{1} \lambda\right)=1$,

(iii) $\varepsilon_{0}(\lambda)=2, \varepsilon_{i}(\lambda)=0$ for $i>0$ and $\widetilde{e}_{0} \lambda \in \operatorname{JS}(0)$.

Then

$$
\operatorname{dim} \operatorname{End}_{\widetilde{\mathrm{S}}_{n-2,2} \cap G}\left(D \downarrow_{\widetilde{\mathrm{S}}_{n-2,2} \cap G}\right)>\operatorname{dim} \operatorname{End}_{\widetilde{\mathrm{S}}_{n-1} \cap G}\left(D \downarrow \widetilde{\mathrm{S}}_{n-1} \cap G\right) .
$$


Proof. We will prove the lemma corresponding to cases (i), (ii) and (iii) separately. We will use Lemma 2.7 without further reference.

Case (i). Notice that $D(\lambda) \downarrow_{\widetilde{\mathrm{S}}_{n-1}} \cong D\left(\widetilde{e}_{1} \lambda\right)^{\oplus 1+a(\lambda)}$ and $D(\lambda) \downarrow_{\widetilde{\mathrm{s}}_{n-2,2}} \cong e_{0} D\left(\widetilde{e}_{1} \lambda\right) \circledast$ $D((2))$. So the lemma holds if $G=\widetilde{\mathrm{S}}_{n}$ and $D \cong D(\lambda, 0)$ or $G=\widetilde{\mathrm{A}}_{n}$ and $D \cong E(\lambda, 0)$ by Lemma 5.9. Assume now that $G=\widetilde{\mathrm{S}}_{n}$ and $D \cong D(\lambda, \pm)$. Then $D(\lambda, \pm) \downarrow_{\widetilde{\mathrm{S}}_{n-1}} \cong$ $D\left(\widetilde{e}_{1} \lambda, 0\right)$ and $D(\lambda, \pm) \downarrow_{\widetilde{S}_{n-2,2}}$ is indecomposable with simple head and socle; it has exactly 3 composition factors of the form $\left(D\left(\widetilde{e}_{0} \widetilde{e}_{1} \lambda,(2)\right),+\right)$ or $\left(D\left(\widetilde{e}_{0} \widetilde{e}_{1} \lambda,(2)\right),-\right)$. Let $b, c \in\{ \pm\}$ such that $D(\lambda, \pm) \downarrow \widetilde{\mathrm{S}}_{n-2,2}$ has a filtration of the form

$$
\left(D\left(\widetilde{e}_{0} \widetilde{e}_{1} \lambda,(2)\right), \pm\right)|\ldots|\left(D\left(\widetilde{e}_{0} \widetilde{e}_{1} \lambda,(2)\right), \pm b\right)|\ldots|\left(D\left(\widetilde{e}_{0} \widetilde{e}_{1} \lambda,(2)\right), \pm c\right) .
$$

Note that by self-duality of $D(\lambda)$ we have that

$$
\left(D(\lambda, \pm) \downarrow_{\widetilde{\mathrm{S}}_{n-2,2}}\right)^{*} \in\left\{D(\lambda, \pm) \downarrow_{\widetilde{\mathrm{S}}_{n-2,2}}, D(\lambda, \mp) \downarrow_{\widetilde{\mathrm{S}}_{n-2,2}}\right\} .
$$

So there exists $d \in\{ \pm\}$ such that $\left(D(\lambda, \pm) \downarrow_{\widetilde{S}_{n-2,2}}\right)^{*}$ has a filtration

$$
\left(D\left(\widetilde{e}_{0} \widetilde{e}_{1} \lambda,(2)\right), \pm c d\right)|\ldots|\left(D\left(\widetilde{e}_{0} \widetilde{e}_{1} \lambda,(2)\right), \pm b d\right)|\ldots|\left(D\left(\widetilde{e}_{0} \widetilde{e}_{1} \lambda,(2)\right), \pm d\right) .
$$

It then follows that $c=+$ and so the lemma holds. The case $G=\widetilde{\mathrm{A}}_{n}$ and $D \cong$ $E(\lambda, \pm)$ holds with similar arguments.

Case (ii). Notice that in this case $\varepsilon_{k}\left(\widetilde{e}_{1} \lambda\right)=0$ for $k \neq 0,2$ since $\lambda \in \operatorname{JS}(1)$ and using [30, Lemma 20.2.3]. In particular $D(\lambda) \downarrow_{\widetilde{\mathrm{s}}_{n-1}} \cong D\left(\widetilde{e}_{1} \lambda\right)^{\oplus 1+a(\lambda)}$ and $D(\lambda) \downarrow_{\widetilde{\mathrm{s}}_{n-2,2}} \cong$ $\left(D\left(\widetilde{e}_{0} \widetilde{e}_{1} \lambda,(2)\right)\right) \oplus\left(D\left(\widetilde{e}_{2} \widetilde{e}_{1} \lambda, D(2)\right)\right)^{\oplus 1+a(\lambda)}$. The lemma then easily follows.

Case (iii). In this case by Lemma 5.15 we have $D(\lambda) \downarrow_{\widetilde{\mathrm{s}}_{n-1}} \cong e_{0} D(\lambda)$ and $D(\lambda) \downarrow_{\widetilde{\mathrm{s}}_{n-2,2}}$ $\cong\left(D\left(\widetilde{e}_{0}^{2} \lambda,(2)\right)\right)^{\oplus 1+a(\lambda)} \oplus A$ with $A \neq 0$ corresponding to blocks different than the block of $D\left(\widetilde{e}_{0}^{2} \lambda,(2)\right)$. So the lemma holds if $G=\widetilde{\mathrm{S}}_{n}$ and $D \cong D(\lambda, 0)$ or $G=\widetilde{\mathrm{A}}_{n}$ and $D \cong E(\lambda, 0)$. Assume now that $G=\widetilde{\mathrm{S}}_{n}$ and $D \cong D(\lambda, \pm)$. Then $D(\lambda, \pm) \downarrow_{\widetilde{\mathrm{S}}_{n-2,2}} \cong\left(D\left(\widetilde{e}_{0}^{2} \lambda,(2)\right), 0\right) \oplus A^{\prime}$ with $A^{\prime} \neq 0$. So it is enough to prove that $\operatorname{dim} \operatorname{End}_{\widetilde{\mathrm{S}}_{n-1}}\left(D(\lambda, \pm) \downarrow_{\widetilde{\mathrm{S}}_{n-1}}\right)=1$. Note that $D(\lambda, \pm) \downarrow_{\widetilde{\mathrm{S}}_{n-1}}$ has simple head and socle and exactly two composition factors of the form $D\left(\widetilde{e}_{0} \lambda,+\right)$ or $D\left(\widetilde{e}_{0} \lambda,-\right)$. Let $b \in\{ \pm\}$ with

$$
D(\lambda, \pm) \downarrow_{\widetilde{\mathrm{s}}_{n-1}} \sim D\left(\widetilde{e}_{0} \lambda, \pm\right)|\ldots| D\left(\widetilde{e}_{0} \lambda, \pm b\right) .
$$

It is enough to prove that $b=-$. This follows from

$$
\begin{aligned}
\operatorname{Res}_{0}\left(D(\lambda, \pm) \downarrow_{\widetilde{\varsigma}_{n-1}}\right) & \cong \operatorname{Res}_{0}^{2}\left(D(\lambda, \pm) \cong \operatorname{Res}_{0}\left(D\left(\widetilde{e}_{0} \lambda, \pm\right) \oplus D\left(\widetilde{e}_{0} \lambda, \pm b\right)\right)\right. \\
& \cong D\left(\widetilde{e}_{0}^{2} \lambda, \pm\right) \oplus D\left(\widetilde{e}_{0}^{2} \lambda, \pm b\right)
\end{aligned}
$$

and from Lemma 5.16. The case $G=\widetilde{\mathrm{A}}_{n}$ and $D \cong E(\lambda, \pm)$ holds similarly.

Lemma 5.18. Let $p \geq 3, n \geq 4$ and $\lambda \in \mathscr{R} \mathscr{P}_{p}(n)$. Assume that $\operatorname{dim} \operatorname{End}_{\widetilde{\boldsymbol{S}}_{n-2,2}}\left(D(\lambda) \downarrow_{\widetilde{\boldsymbol{S}}_{n-2,2}}\right)>\operatorname{dim} \operatorname{End}_{\widetilde{\boldsymbol{S}}_{n-1}}\left(D(\lambda) \downarrow_{\widetilde{\mathrm{S}}_{n-1}}\right)+\operatorname{dim} \operatorname{End}_{\widetilde{\boldsymbol{S}}_{n}}(D(\lambda))$. 
Then

- If $D(\lambda)$ is of type $M$ then there exists

$$
\psi \in \operatorname{Hom}_{\widetilde{\mathrm{S}}_{n}}\left(M_{2}, \operatorname{End}_{F}(D(\lambda, 0))\right)
$$

which does not vanish on $S_{2}$. Further there exist

$$
\varphi_{1}, \varphi_{2} \in \operatorname{Hom}_{\widetilde{A}_{n}}\left(M_{2}, \operatorname{Hom}_{F}(E(\lambda, \pm), E(\lambda))\right)
$$

which are linearly independent over $S_{2}$.

- If $D(\lambda)$ is of type $Q$ then there exist

$$
\psi_{1}, \psi_{2} \in \operatorname{Hom}_{\widetilde{\mathbf{S}}_{n}}\left(M_{2}, \operatorname{Hom}_{F}(D(\lambda, \pm), D(\lambda))\right)
$$

which are linearly independent over $S_{2}$. Further there exists

$$
\varphi \in \operatorname{Hom}_{\widetilde{\mathrm{A}}_{n}}\left(M_{2}, \operatorname{End}_{F}(E(\lambda, 0))\right)
$$

which does not vanish on $S_{2}$.

Proof. From Lemma 4.1 we have that $M_{2} \sim S_{2} \mid M_{1}$.

Assume first that $D(\lambda)$ is of type $\mathrm{M}$, so that

$$
\operatorname{dim} \operatorname{End}_{\widetilde{\mathbf{S}}_{n-2,2}}\left(D(\lambda) \downarrow_{\widetilde{\mathbf{S}}_{n-2,2}}\right)>\operatorname{dim} \operatorname{End}_{\widetilde{\boldsymbol{S}}_{n-1}}\left(D(\lambda) \downarrow_{\widetilde{\mathbf{S}}_{n-1}}\right)+1 .
$$

Since $D(\lambda) \downarrow_{\widetilde{\mathrm{A}}_{n}} \cong E(\lambda)$ and $D(\lambda, 0) \cong D(\lambda) \cong E(\lambda, \pm) \uparrow^{\widetilde{S}_{n}}$, for any partition $\mu \neq$ $\left(1^{n}\right)$ we have that

$$
\operatorname{dim} \operatorname{End}_{\widetilde{\mathrm{S}}_{\mu}}\left(D(\lambda) \downarrow_{\widetilde{\mathrm{S}}_{\mu}}\right)=\operatorname{dim} \operatorname{End}_{\widetilde{\mathrm{A}}_{\mu}}\left(E(\lambda, \pm) \downarrow_{\widetilde{\mathrm{A}}_{\mu}}, E(\lambda) \downarrow_{\widetilde{\mathrm{A}}_{\mu}}\right) .
$$

The lemma then easily follows in this case.

Assume next that $D(\lambda)$ is of type $\mathrm{Q}$, so that

$$
\operatorname{dim} \operatorname{End}_{\widetilde{\mathbf{S}}_{n-2,2}}\left(D(\lambda) \downarrow_{\widetilde{\mathbf{S}}_{n-2,2}}\right)>\operatorname{dim} \operatorname{End}_{\widetilde{\mathbf{S}}_{n-1}}\left(D(\lambda) \downarrow_{\widetilde{\mathbf{S}}_{n-1}}\right)+2 .
$$

Then for some $\varepsilon \in\{ \pm\}$ we have that

$$
\begin{aligned}
& \operatorname{dim} \operatorname{Hom}_{\widetilde{\mathrm{S}}_{n-2,2}}\left(D(\lambda, \varepsilon) \downarrow_{\widetilde{\mathrm{S}}_{n-2,2}}, D(\lambda) \downarrow_{\widetilde{\mathrm{S}}_{n-2,2}}\right) \\
& \geq \operatorname{dim} \operatorname{Hom}_{\widetilde{\mathrm{S}}_{n-1}}\left(D(\lambda, \varepsilon) \downarrow_{\widetilde{\mathrm{S}}_{n-1}}, D(\lambda) \downarrow_{\widetilde{\mathrm{S}}_{n-1}}\right)+2 .
\end{aligned}
$$

So there exist $\psi_{1}, \psi_{2} \in \operatorname{Hom}_{\widetilde{\mathrm{S}}_{n}}\left(M_{2}, \operatorname{Hom}_{F}(D(\lambda, \varepsilon), D(\lambda))\right)$ which are linearly independent over $S_{2}$. The lemma then follows from

$$
D(\lambda,+) \otimes D(\lambda) \cong D(\lambda,+) \otimes \operatorname{sgn} \otimes D(\lambda) \cong D(\lambda, \varepsilon) \otimes D(\lambda)
$$

and from $D(\lambda, \pm) \downarrow_{\widetilde{\mathrm{A}}_{n}} \cong E(\lambda, 0)$.

Lemma 5.19. Let $p \geq 3, n \geq 4$ and $\lambda \in \mathscr{R} \mathscr{P}_{p}(n)$. Assume that $\lambda \neq \beta_{n}$ and $\lambda$ is not JS(0). Then:

- If $D(\lambda)$ is of type $M$ then there exists

$$
\psi \in \operatorname{Hom}_{\widetilde{\mathrm{S}}_{n}}\left(M_{2}, \operatorname{End}_{F}(D(\lambda, 0))\right)
$$

which does not vanish on $S_{2}$. Further there exists

$$
\varphi \in \operatorname{Hom}_{\widetilde{\mathrm{A}}_{n}}\left(M_{2}, \operatorname{End}_{F}(E(\lambda, \pm))\right)
$$

which does not vanish on $S_{2}$ or there exist

$$
\varphi_{1}, \varphi_{2} \in \operatorname{Hom}_{\widetilde{\mathrm{A}}_{n}}\left(M_{2}, \operatorname{Hom}_{F}(E(\lambda, \pm), E(\lambda, \mp))\right)
$$

which are linearly independent over $S_{2}$. 
- If $D(\lambda)$ is of type $Q$ then there exists

$$
\psi \in \operatorname{Hom}_{\widetilde{\mathrm{S}}_{n}}\left(M_{2}, \operatorname{End}_{F}(D(\lambda, \pm))\right)
$$

which does not vanish on $S_{2}$ or there exist

$$
\psi_{1}, \psi_{2} \in \operatorname{Hom}_{\widetilde{\mathrm{S}}_{n}}\left(M_{2}, \operatorname{Hom}_{F}(D(\lambda, \pm), D(\lambda, \mp))\right)
$$

which are linearly independent over $S_{2}$. Further there exists

$$
\varphi \in \operatorname{Hom}_{\widetilde{\mathrm{A}}_{n}}\left(M_{2}, \operatorname{End}_{F}(E(\lambda, 0))\right)
$$

which does not vanish on $S_{2}$.

Proof. From Lemma 5.18 we may assume that

$$
\operatorname{dim} \operatorname{End}_{\widetilde{\mathrm{S}}_{n-2,2}}\left(D(\lambda) \downarrow_{\widetilde{\mathrm{S}}_{n-2,2}}\right) \leq \operatorname{dim} \operatorname{End}_{\widetilde{\mathrm{S}}_{n-1}}\left(D(\lambda) \downarrow_{\widetilde{\mathrm{S}}_{n-1}}\right)+\operatorname{dim} \operatorname{End}_{\widetilde{\mathrm{S}}_{n}}(D(\lambda)) .
$$

Let $G \in\left\{\widetilde{\mathrm{S}}_{n}, \widetilde{\mathrm{A}}_{n}\right\}$ and $D$ be an $F G$-representation indexed by $\lambda$. Then by Lemmas 5.14 and 5.17 we have that

$$
\operatorname{dim} \operatorname{End}_{\widetilde{\mathrm{S}}_{n-2,2} \cap G}\left(D \downarrow_{\widetilde{\mathrm{S}}_{n-2,2} \cap G}\right)>\operatorname{dim} \operatorname{End}_{\widetilde{\mathrm{S}}_{n-1} \cap G}\left(D \downarrow_{\widetilde{\mathrm{S}}_{n-1} \cap G}\right) .
$$

Since $M_{2} \sim S_{2} \mid M_{1}$ by Lemma 4.1, the lemma easily follows.

\subsection{Basic spin modules.}

Lemma 5.20. Let $p \geq 3$. Let $c=1$ if $p \nmid n$ or $c=2$ if $p \mid n$.

- If $D\left(\beta_{n}\right)$ is of type $M$ then $D\left(\beta_{n}, 0\right) \otimes D\left(\beta_{n}\right) \cong \oplus_{k=0}^{n-c} \bar{D}_{k}$ and $E\left(\beta_{n}, \pm\right) \otimes E\left(\beta_{n}\right) \cong \bar{E}_{(n-c) / 2, \pm} \oplus_{k=0}^{(n-2-c) / 2} \bar{E}_{k}$.

- If $D\left(\beta_{n}\right)$ is of type $Q$ then $D\left(\beta_{n}, \pm\right) \otimes D\left(\beta_{n}\right) \cong \oplus_{k=0}^{n-c} \bar{D}_{k}$ and $E\left(\beta_{n}, 0\right) \otimes E\left(\beta_{n}\right) \cong \oplus_{k=0}^{(n-1-c) / 2} \bar{E}_{k}$.

Proof. Note that by [4, Theorem 9.3]

$$
[S((n), \varepsilon) \otimes S((n))]=\sum_{k=0}^{n-1}\left[S^{\left(n-k, 1^{k}\right)}\right],
$$

with $\varepsilon=0$ or \pm depending on the type of $S((n))$. Let $\varepsilon^{\prime}=0$ or \pm depending on the type of $D\left(\beta_{n}\right)$. By Lemma 2.18 we have that

$$
[S((n), \varepsilon) \otimes S((n))]=\left(1+\delta_{p \mid n}\right)\left[D\left(\beta_{n}, \varepsilon^{\prime}\right) \otimes D\left(\beta_{n}\right)\right] .
$$

Further from Lemma 2.23

$$
\sum_{k=0}^{n-1}\left[S^{\left(n-k, 1^{k}\right)}\right]=\left(1+\delta_{p \mid n}\right) \sum_{k=0}^{n-c}\left[\bar{D}_{k}\right] .
$$

So

$$
\left[D\left(\beta_{n}, \varepsilon^{\prime}\right) \otimes D\left(\beta_{n}\right)\right]=\sum_{k=0}^{n-c}\left[\bar{D}_{k}\right] .
$$

Since $D\left(\beta_{n}, \varepsilon^{\prime}\right) \otimes \operatorname{sgn} \cong D\left(\beta_{n},-\varepsilon^{\prime}\right)$, we have that

$$
D\left(\beta_{n}, \varepsilon^{\prime}\right) \otimes D\left(\beta_{n}\right) \cong D\left(\beta_{n},-\varepsilon^{\prime}\right) \otimes \operatorname{sgn} \otimes D\left(\beta_{n}\right) \cong D\left(\beta_{n},-\varepsilon^{\prime}\right) \otimes D\left(\beta_{n}\right) .
$$

From $D\left(\beta_{n}\right)$ being self-dual it then follows that so is $D\left(\beta_{n}, \varepsilon^{\prime}\right) \otimes D\left(\beta_{n}\right)$. Since this module is self-dual, multiplicity free and its composition factors are self-dual, the lemma holds for $\widetilde{\mathrm{S}}_{n}$. For $\widetilde{\mathrm{A}}_{n}$ the lemma then follows by Lemma 2.24. 
Lemma 5.21. Let $p \geq 3$ and $n \geq 10$. Then $\bar{D}_{2} \subseteq \operatorname{End}_{F}\left(D\left(\beta_{n}, \delta\right)\right)$ and $\bar{E}_{2} \subseteq$ $\operatorname{End}_{F}\left(E\left(\beta_{n}, \delta^{\prime}\right)\right)$.

Proof. In this case it can be easily checked from Lemma 2.24 that $\bar{D}_{2} \cong D_{1^{2}}$ and that $\left(n-2,1^{2}\right)>\left(n-2,1^{2}\right)^{\mathrm{M}}$. We will use Lemma 2.7 without further reference.

Note that any composition factor (as supermodule) of $D\left(\beta_{n}\right) \downarrow_{\widetilde{S}_{n-k}}$ is of the form $D\left(\beta_{n-k}\right)$ (this holds for example by Lemma 2.18 and branching in characteristic $0)$. So any composition factor of $D\left(\beta_{n}\right) \downarrow_{\widetilde{S}_{\alpha}}$ is of the form $D\left(\beta_{\alpha_{1}}, \beta_{\alpha_{2}}, \ldots\right)$.

Consider first $D\left(\beta_{n}, \delta\right)$. If $\delta=0$ then $D_{1^{2}} \subseteq \operatorname{End}_{F}\left(D\left(\beta_{n}, \delta\right)\right)$ by Lemma 5.20. So we may assume that $\delta= \pm$. If $n \not \equiv 0,1,2 \bmod p$ then

$$
\begin{aligned}
& D\left(\beta_{n}\right) \downarrow_{\widetilde{\mathrm{s}}_{n-1}} \cong D\left(\beta_{n-1}\right)^{\oplus 2}, \\
& D\left(\beta_{n}\right) \downarrow_{\widetilde{\mathrm{s}}_{n-2}} \cong D\left(\beta_{n-2}\right)^{\oplus 2}, \\
& D\left(\beta_{n}\right) \downarrow_{\widetilde{\mathrm{s}}_{n-2,2}} \cong D\left(\beta_{n-2},(2)\right)^{\oplus 2},
\end{aligned}
$$

with $D\left(\beta_{n-1}\right)$ and $D\left(\beta_{n-2},(2)\right)$ of type $\mathrm{M}$ and $D\left(\beta_{n-2}\right)$ of type Q. So $D\left(\beta_{n}, \pm\right) \downarrow \widetilde{s}_{n-1}$ and $D\left(\beta_{n}, \pm\right) \downarrow_{\widetilde{S}_{n-2,2}}$ are simple, while $D\left(\beta_{n}, \pm\right) \downarrow_{\widetilde{S}_{n-2}}$ is a direct sum of two simple modules. So $D_{1^{2}} \subseteq \operatorname{End}_{F}\left(D\left(\beta_{n}, \delta\right)\right)$ by Lemmas 2.20 and 4.4 .

If $n \equiv 2 \bmod p$ then

$$
\begin{aligned}
& D\left(\beta_{n}\right) \downarrow_{\widetilde{\mathrm{s}}_{n-1}} \cong D\left(\beta_{n-1}\right)^{\oplus 2}, \\
& D\left(\beta_{n}\right) \downarrow_{\widetilde{\mathrm{S}}_{n-2}} \cong\left(D\left(\beta_{n-2}\right) \mid D\left(\beta_{n-2}\right)\right)^{\oplus 2}, \\
& D\left(\beta_{n}\right) \downarrow_{\widetilde{\mathrm{s}}_{n-2,2}} \cong D\left(\beta_{n-2},(2)\right) \mid D\left(\beta_{n-2},(2)\right), \\
& D\left(\beta_{n}\right) \downarrow_{\widetilde{\mathrm{s}}_{n-3,2}} \cong D\left(\beta_{n-3},(2)\right)^{\oplus 2},
\end{aligned}
$$

with $D\left(\beta_{n-1}\right), D\left(\beta_{n-2}\right)$ and $D\left(\beta_{n-3},(3)\right)$ of type $\mathrm{M}$ and $D\left(\beta_{n-2},(2)\right)$ and $D\left(\beta_{n-3}\right.$, (2)) of type Q. In particular $D\left(\beta_{n},+\right) \downarrow_{\widetilde{S}_{n-1}} \cong D\left(\beta_{n},-\right) \downarrow_{\widetilde{S}_{n-1}}$ are simple, $D\left(\beta_{n}, \pm\right) \downarrow \widetilde{s}_{n-2}$ is uniserial with two isomorphic composition factors and $D\left(\beta_{n}, \pm\right) \downarrow_{\widetilde{S}_{n-2,2}}$ is uniserial with two non-isomorphic composition factors (since $D\left(\beta_{n},+\right) \downarrow_{\widetilde{\mathrm{s}}_{n-1}} \cong D\left(\beta_{n},-\right) \downarrow_{\widetilde{\mathrm{s}}_{n-1}}$ the two composition factors of $D\left(\beta_{n}, \pm\right) \downarrow_{\widetilde{s}_{n-3,2}}$ are not isomorphic). It then follows again by Lemmas 2.20 and 4.4 that $D_{1^{2}} \subseteq \operatorname{End}_{F}\left(D\left(\beta_{n}, \delta\right)\right)$.

If $n \equiv 1 \bmod p$ then

$$
\begin{aligned}
& D\left(\beta_{n}\right) \downarrow_{\widetilde{\mathrm{s}}_{n-1}} \cong D\left(\beta_{n-1}\right) \mid D\left(\beta_{n-1}\right), \\
& D\left(\beta_{n}\right) \downarrow_{\widetilde{\mathrm{s}}_{n-2}} \cong\left(D\left(\beta_{n-2}\right)\right)^{\oplus 2}, \\
& D\left(\beta_{n}\right) \downarrow_{\widetilde{\mathrm{s}}_{n-2,2}} \cong D\left(\beta_{n-2},(2)\right)^{\oplus 2},
\end{aligned}
$$

with $D\left(\beta_{n-1}\right)$ and $D\left(\beta_{n-2}\right)$ of type $\mathrm{Q}$ and $D\left(\beta_{n-2},(2)\right)$ of type M. In particular $D\left(\beta_{n},+\right) \downarrow_{\widetilde{\mathrm{S}}_{n-2,2}} \cong D\left(\beta_{n},-\right) \downarrow_{\widetilde{\mathrm{S}}_{n-2,2}}$ are simple, from which it follows that $D\left(\beta_{n}, \pm\right) \downarrow_{\widetilde{S}_{n-2}} \cong D\left(\beta_{n-2},+\right) \oplus D\left(\beta_{n-2},-\right)$ and then that

$$
D\left(\beta_{n}\right) \downarrow_{\widetilde{S}_{n-1}} \cong D\left(\beta_{n-1}, \pm\right) \mid D\left(\beta_{n-1}, \mp\right),
$$

so again $D_{1^{2}} \subseteq \operatorname{End}_{F}\left(D\left(\beta_{n}, \delta\right)\right)$. 
If $n \equiv 0 \bmod p$ and $p \neq 3$ then

$$
\begin{aligned}
& D\left(\beta_{n}\right) \downarrow_{\widetilde{\mathrm{s}}_{n-3}} \cong D\left(\beta_{n-3}\right)^{\oplus 2}, \\
& D\left(\beta_{n}\right) \downarrow_{\widetilde{\mathrm{S}}_{n-3,2}} \cong D\left(\beta_{n-3},(2)\right)^{\oplus 2}, \\
& D\left(\beta_{n}\right) \downarrow_{\widetilde{\mathrm{s}}_{n-3,3}} \cong D\left(\beta_{n-3},(3)\right),
\end{aligned}
$$

with $D\left(\beta_{n-3}\right)$ and $D\left(\beta_{n-3},(3)\right)$ of type $\mathrm{Q}$, while $D\left(\beta_{n-3},(2)\right)$ is of type M. So $D\left(\beta_{n}, \pm\right) \downarrow_{\widetilde{\mathrm{S}}_{n-3,2}}$ and $D\left(\beta_{n}, \pm\right) \downarrow_{\widetilde{\mathrm{S}}_{n-3,3}}$ are simple, while $D\left(\beta_{n}, \pm\right) \downarrow_{\widetilde{\mathrm{S}}_{n-3}}$ is a direct sum of two simple modules. Then $D_{1^{2}} \subseteq \operatorname{End}_{F}\left(D\left(\beta_{n}, \delta\right)\right)$ by Lemmas 2.20 and 4.5, since $\operatorname{End}_{F}\left(D\left(\beta_{n}, \delta\right)\right)$ is semisimple by Lemma 5.20 .

If $n \equiv 0 \bmod p$ and $p=3$ then

$$
\begin{aligned}
& D\left(\beta_{n}\right) \downarrow_{\widetilde{\mathrm{s}}_{n-1}} \cong D\left(\beta_{n-1}\right), \\
& D\left(\beta_{n}\right) \downarrow_{\widetilde{\mathrm{s}}_{n-2}} \cong D\left(\beta_{n-2}\right)^{\oplus 2}, \\
& D\left(\beta_{n}\right) \downarrow_{\widetilde{\mathrm{s}}_{n-3}} \cong\left(D\left(\beta_{n-3}\right) \mid D\left(\beta_{n-3}\right)\right)^{\oplus 2}, \\
& D\left(\beta_{n}\right) \downarrow_{\widetilde{\mathrm{s}}_{n-3,2}} \cong D\left(\beta_{n-3},(2)\right) \mid D\left(\beta_{n-3},(2)\right), \\
& D\left(\beta_{n}\right) \downarrow_{\widetilde{\mathrm{s}}_{n-3,3}} \cong D\left(\beta_{n-3},(2,1)\right) \mid D\left(\beta_{n-3},(2,1)\right), \\
& D\left(\beta_{n}\right) \downarrow_{\widetilde{\mathrm{s}}_{n-4,2}} \cong D\left(\beta_{n-4},(2)\right)^{\oplus 2} .
\end{aligned}
$$

Further $D\left(\beta_{n-2}\right)$ and $D\left(\beta_{n-3}\right)$ are of type $\mathrm{M}$ while $D\left(\beta_{n-1}\right), D\left(\beta_{n-3},(2)\right), D\left(\beta_{n-3}\right.$, $(2,1))$ and $D\left(\beta_{n-4},(2)\right)$ are of type $\mathrm{Q}$. In particular $D\left(\beta_{n},+\right) \downarrow_{\widetilde{\mathrm{S}}_{n-2}} \cong D\left(\beta_{n},+\right) \downarrow_{\widetilde{\mathrm{S}}_{n-2}}$, from which it follows that

$$
D\left(\beta_{n},+\right) \downarrow_{\widetilde{\mathrm{s}}_{n-4,2}} \cong D\left(\beta_{n-4},(2),+\right) \oplus D\left(\beta_{n-4},(2),-\right) .
$$

So

$$
\begin{aligned}
& D\left(\beta_{n}, \pm\right) \downarrow_{\widetilde{\mathrm{s}}_{n-3}} \cong D\left(\beta_{n-3}, 0\right) \mid D\left(\beta_{n-3}, 0\right), \\
& D\left(\beta_{n}, \pm\right) \downarrow_{\widetilde{\mathrm{S}}_{n-3,2}} \cong D\left(\beta_{n-3},(2), \pm\right) \mid D\left(\beta_{n-3},(2), \mp\right), \\
& D\left(\beta_{n}, \pm\right) \downarrow_{\widetilde{\mathrm{s}}_{n-3,3}} \cong D\left(\beta_{n-3},(2,1), \pm\right) \mid D\left(\beta_{n-3},(2,1), \mp\right) .
\end{aligned}
$$

Since $\operatorname{End}_{F}\left(D\left(\beta_{n}, \delta\right)\right)$ is semisimple by Lemma 5.20, it follows from Lemma 4.5 that $D_{1^{2}} \subseteq \operatorname{End}_{F}\left(D\left(\beta_{n}, \delta\right)\right)$.

For $\widetilde{\mathrm{A}}_{n}$ the proof is similar (it uses the restriction to the corresponding subgroups of $\left.\widetilde{\mathrm{A}}_{n}\right)$.

\section{Tensor products}

In this section we will consider tensor products with special classes of modules. In order to check if tensor products are irreducible we will at times use the following lemmas.

Lemma 6.1. Let $D$ be an irreducible $F \widetilde{\mathrm{S}}_{n}$-module and $\mu \in \mathscr{R} \mathscr{P}_{p}(n)$. If $D \otimes D(\lambda, \delta)$ is irreducible then

$$
\operatorname{dim} \operatorname{Hom}_{\widetilde{\mathrm{S}}_{n}}\left(\operatorname{End}_{F}(D), \operatorname{Hom}_{F}(D(\mu), D(\mu, \delta)) \leq 1+a(\mu) .\right.
$$

Similarly if $E$ is an irreducible $F \widetilde{\mathrm{A}}_{n}$-module, $\mu \in \mathscr{R} \mathscr{P}_{p}(n)$ and $E \otimes D\left(\lambda, \delta^{\prime}\right)$ is irreducible then

$$
\operatorname{dim} \operatorname{Hom}_{\widetilde{\mathrm{A}}_{n}}\left(\operatorname{End}_{F}(E), \operatorname{Hom}_{F}\left(E(\mu), E\left(\mu, \delta^{\prime}\right)\right) \leq 2-a(\mu) .\right.
$$


Proof. Similar to [7, Lemma 3.4].

Lemma 6.2. Let $\lambda \in \mathscr{P}_{p}(n)$ and $\mu \in \mathscr{R} \mathscr{P}_{p}(n)$. If $D(\mu)$ is of type $Q$ and $\operatorname{dim} \operatorname{Hom}_{\widetilde{\mathrm{S}}_{n}}\left(\operatorname{End}_{F}\left(D^{\lambda}\right), \operatorname{Hom}_{F}(D(\mu), D(\mu, \pm))=2\right.$

then

- if $D^{\lambda} \otimes D(\mu)$ has a composition factor of type $M$ then $D^{\lambda} \otimes D(\mu, \pm)$ is irreducible,

- if $D^{\lambda} \otimes D(\mu)$ has a composition factor of type $Q$ then $D^{\lambda} \otimes D(\mu, \pm)$ is not irreducible.

Similarly if $\lambda \in \mathscr{P}_{p}(n) \backslash \mathscr{P}_{p}^{A}(n), D(\mu)$ is of type $M$ and

$$
\operatorname{dim} \operatorname{Hom}_{\widetilde{\mathrm{A}}_{n}}\left(\operatorname{End}_{F}\left(E^{\lambda}\right), \operatorname{Hom}_{F}(E(\mu), E(\mu, \pm))=2\right.
$$

then

- if $D^{\lambda} \otimes D(\mu)$ has a composition factor of type $M$ then $E^{\lambda} \otimes E(\mu, \pm)$ is not irreducible,

- if $D^{\lambda} \otimes D(\mu)$ has a composition factor of type $Q$ then $E^{\lambda} \otimes E(\mu, \pm)$ is irreducible.

Proof. We will prove the lemma only for $\widetilde{\mathrm{S}}_{n}$, the proof for $\widetilde{\mathrm{A}}_{n}$ being similar (using conjugation by elements in $\widetilde{\mathrm{S}}_{n} \backslash \widetilde{\mathrm{A}}_{n}$ instead of tensoring with $\left.\operatorname{sgn}\right)$.

As $D(\mu)=D(\mu,+) \oplus D(\mu,-)$ and $D(\mu,+) \cong D(\mu,-) \otimes \operatorname{sgn}$,

$$
\begin{aligned}
\operatorname{dim} \operatorname{End}_{\widetilde{\mathrm{S}}_{n}}\left(D^{\lambda} \otimes D(\mu)\right) & =\operatorname{dim} \operatorname{Hom}_{\widetilde{\mathrm{S}}_{n}}\left(\operatorname{End}_{F}\left(D^{\lambda}\right), \operatorname{End}_{F}(D(\mu))\right. \\
& =2 \operatorname{dim}_{\widetilde{\mathrm{S}}_{n}}\left(\operatorname{End}_{F}\left(D^{\lambda}\right), \operatorname{Hom}_{F}(D(\mu), D(\mu, \pm))\right. \\
& =4 .
\end{aligned}
$$

Let $D(\nu) \subseteq D^{\lambda} \otimes D(\mu)$. Assume first that $D(\nu)$ is of type M. Then $D(\nu)=D(\nu, 0) \cong$ $D(\nu, 0) \otimes \operatorname{sgn}$. From $D(\mu,+) \cong D(\mu,-) \otimes \operatorname{sgn}$ it follows that $D(\nu)^{\oplus 2} \subseteq D^{\lambda} \otimes D(\mu)$. Since $D^{\lambda} \otimes D(\mu)$ is self-dual and so it has isomorphic head and socle, it follows that $D^{\lambda} \otimes D(\mu) \cong D(\nu)^{\oplus 2}$. In particular module $D^{\lambda} \otimes D(\mu)$ has exactly two composition factors and so $D^{\lambda} \otimes D(\mu, \pm)$ is irreducible.

Assume now that $D(\nu)$ is of type Q. Then $D^{\lambda} \otimes D(\mu) \nRightarrow D(\nu)$. In particular module $D^{\lambda} \otimes D(\mu)$ has more than two composition factors. Since $D^{\lambda} \otimes D(\mu,+) \cong$ $\left(D^{\lambda} \otimes D(\mu,-)\right) \otimes \operatorname{sgn}$, it then follows that $D^{\lambda} \otimes D(\mu, \pm)$ is not irreducible in this case.

\subsection{Tensor products with natural modules.}

Lemma 6.3. Let $n \geq 4, G=\widetilde{\mathrm{S}}_{n}$ or $\widetilde{\mathrm{A}}_{n}, \lambda \in \mathscr{R} \mathscr{P}_{p}(n)$ and $V$ be a simple spin $G$-module indexed by $\lambda$. If $V \otimes D^{(n-1,1)} \downarrow_{G}$ is simple then, as supermodule,

$$
\left[D(\lambda) \otimes M_{1}: D(\lambda)\right]= \begin{cases}1, & n \neq \equiv 0 \bmod p \\ 2, & n \equiv 0 \bmod p .\end{cases}
$$

Proof. Since $n \geq 4$ we have that $D^{(n-1,1)} \downarrow_{G}$ has dimension greater than 1 . Let $V^{\prime}$ be any simple spin $G$-module indexed by $\lambda$. Then $V^{\prime} \otimes D^{(n-1,1)} \downarrow_{G}$ is simple (by either tensoring with sgn if $G=\widetilde{\mathrm{S}}_{n}$ or conjugating with $\sigma \in \widetilde{\mathrm{S}}_{n} \backslash \widetilde{\mathrm{A}}_{n}$ if $G=\widetilde{\mathrm{A}}_{n}$ ) and so $V$ is not a composition factor of $V^{\prime} \otimes D^{(n-1,1)} \downarrow_{G}$. So $\left[D(\lambda) \otimes M_{1}: D(\lambda)\right]=\left[M_{1}: D_{0}\right]$ and then the lemma holds by Lemma 4.2

Lemma 6.4. Let $G=\widetilde{\mathrm{S}}_{n}$ or $\widetilde{\mathrm{A}}_{n}$ and $\lambda \in \mathscr{R} \mathscr{P}_{p}(n)$. 
- If $G=\widetilde{\mathrm{S}}_{n}$ and $D(\lambda)$ is of type $M$ then $D(\lambda, 0) \otimes D^{(n-1,1)}$ is irreducible if and only if as supermodule $D(\lambda) \otimes D^{(n-1,1)}$ is irreducible of type $M$.

- If $G=\widetilde{\mathrm{S}}_{n}$ and $D(\lambda)$ is of type $Q$ then $D(\lambda, \pm) \otimes D^{(n-1,1)}$ is irreducible if and only if as supermodule $D(\lambda) \otimes D^{(n-1,1)}$ is irreducible of type $Q$ or it has exactly two composition factors both of type $M$.

- If $G=\widetilde{\mathrm{A}}_{n}$ then $E(\lambda, 0) \otimes E^{(n-1,1)}$ or $E(\lambda, \pm) \otimes E^{(n-1,1)}$ is irreducible if and only if as supermodule $D(\lambda) \otimes D^{(n-1,1)}$ is irreducible.

Proof. This holds by comparing the number of composition factors of $D(\lambda) \downarrow_{G}$ and of $\left(D(\lambda) \otimes D^{(n-1,1)}\right) \downarrow_{G}$.

Theorem 6.5. Let $n \geq 4, G=\widetilde{\mathrm{S}}_{n}$ or $\widetilde{\mathrm{A}}_{n}, \lambda \in \mathscr{R} \mathscr{P} p(n)$ and $V$ be a simple spin $G$-module indexed by $\lambda$. If $V \otimes D^{(n-1,1)} \downarrow_{G}$ is simple then $n \not \equiv 0 \bmod p$ and $\lambda \in J S(0)$.

In this case, if $\nu=(\lambda \backslash A) \cup B$ where $A$ is the bottom removable node of $\lambda$ and $B$ is the top addable node of $\lambda$,

- if $D(\lambda)$ is of type $M$ then $D(\lambda, 0) \otimes D^{(n-1,1)}$ is not irreducible, while $E(\lambda, \pm) \otimes E^{(n-1,1)} \cong E(\nu, 0)$,

- if $D(\lambda)$ is of type $Q$ then $D(\lambda, \pm) \otimes D^{(n-1,1)} \cong D(\nu, 0)$, while $E(\lambda, 0) \otimes$ $E^{(n-1,1)}$ is not irreducible.

Proof. Let $c:=1$ if $D(\lambda)$ is of type M or $c:=2$ if $D(\lambda)$ is of type Q. Assume that $V \otimes D^{(n-1,1)} \downarrow_{G}$ is simple. We will use Lemmas 2.7 and 2.8 without further notice.

Case 1. $n \equiv 0 \bmod p$. From Lemma 2.13 we have that $\varepsilon_{0}(\lambda)+\varphi_{0}(\lambda)$ is odd. So by Lemmas 2.12 and 6.3 we have that $\lambda \in J S(i)$ and $\varphi_{i}(\lambda)=0$ for some $i \geq 1$. Note that

$$
\begin{aligned}
D(\lambda) \otimes M_{1} & \cong\left(f_{i} e_{i} D(\lambda)\right)^{\oplus 2} \oplus \sum_{j \geq 1: j \neq i}\left(f_{j} e_{i} D(\lambda)\right)^{\oplus 2} \oplus\left(f_{0} e_{i} D(\lambda)\right)^{\oplus c} \\
& \cong D(\lambda)^{\oplus 2} \oplus \sum_{j \geq 1: j \neq i}\left(f_{j} D\left(\widetilde{e}_{i} \lambda\right)\right)^{\oplus 2} \oplus\left(f_{0} D\left(\widetilde{e}_{i} \lambda\right)\right)^{\oplus c} .
\end{aligned}
$$

It then follows from Lemma 4.2 and considering block decomposition that

$$
D(\lambda) \otimes D^{(n-1,1)} \cong \sum_{j \geq 1: j \neq i}\left(f_{j} D\left(\widetilde{e}_{i} \lambda\right)\right)^{\oplus 2} \oplus\left(f_{0} D\left(\widetilde{e}_{i} \lambda\right)\right)^{\oplus c} .
$$

By Lemma 6.4 it follows that if $D(\lambda)$ is of type $\mathrm{Q}$ then it needs to have exactly two composition factors of type $\mathrm{M}$, while if $D(\lambda)$ is of type $\mathrm{M}$ then $D(\lambda) \otimes D_{1}$ is irreducible as supermodule. In either case $\varphi_{i}\left(\widetilde{e}_{i} \lambda\right)=\varphi_{0}\left(\widetilde{e}_{i} \lambda\right)=1$ and $\varphi_{j}\left(\widetilde{e}_{i} \lambda\right)=0$ for $j \neq 0, i$.

In particular $D(\lambda) \otimes M_{1} \cong D(\lambda)^{\oplus 2} \oplus D\left(\widetilde{f}_{0} \widetilde{e}_{i} \lambda\right)^{\oplus c}$. Notice also that from Lemma 2.13 either $\varphi_{0}(\lambda)=3$ and $\varphi_{k}(\lambda)=0$ else or there exists $j \neq 0, i$ such that $\varphi_{0}(\lambda)=$ $\varphi_{j}(\lambda)=1$ and $\varphi_{k}(\lambda)=0$ else.

Case 1.1. $\varphi_{0}(\lambda)=3$ and $\varphi_{j}(\lambda)=0$ else.

From Lemma 2.14

$$
D\left(\widetilde{f}_{0} \widetilde{e}_{i} \lambda\right)^{\oplus c} \cong \operatorname{Ind}_{0} \operatorname{Res}_{i} D(\lambda) \cong \operatorname{Res}_{i} \operatorname{Ind}_{0} D(\lambda) \cong \operatorname{Res}_{i} f_{0} D(\lambda)
$$

and

$$
0=\operatorname{Ind}_{0} \operatorname{Res}_{j} D(\lambda) \cong \operatorname{Res}_{j} \operatorname{Ind}_{0} D(\lambda) \cong \operatorname{Res}_{j} f_{0} D(\lambda)
$$


for $j \neq 0, i$. Since $c \leq 2<\left[f_{0} D(\lambda): D\left(\tilde{f}_{0} \lambda\right)\right]=\varphi_{0}(\lambda)=3$, it follows that $\tilde{f}_{0}$ has only normal nodes of residue 0 and then $\widetilde{f}_{0} \lambda \in J S(0)$, since $\varepsilon_{0} \lambda=0$. Since $\varphi_{0}\left(\widetilde{f}_{0} \lambda\right)=2$ we have from Lemma 5.11 that $n+1 \equiv 0 \bmod p$, leading to a contradiction.

Case 1.2. There exists $j \neq 0, i$ such that $\varphi_{0}(\lambda)=\varphi_{j}(\lambda)=1$ and $\varphi_{k}(\lambda)=0$ else. In this case by Lemma 2.14

$$
\operatorname{Res}_{i} D\left(\widetilde{f}_{j} \lambda\right)^{\oplus c} \cong \operatorname{Res}_{i} \operatorname{Ind}_{j} D(\lambda) \cong \operatorname{Ind}_{j} \operatorname{Res}_{i} D(\lambda) \cong \operatorname{Ind}_{j} D\left(\widetilde{e}_{i} \lambda\right)^{\oplus c}=0
$$

and

$$
\operatorname{Res}_{k} D\left(\tilde{f}_{j} \lambda\right)^{\oplus c} \cong \operatorname{Res}_{k} \operatorname{Ind}_{j} D(\lambda) \cong \operatorname{Ind}_{j} \operatorname{Res}_{k} D(\lambda)=0
$$

for $k \neq i, j$. So all normal nodes of $\widetilde{f}_{j} \lambda$ have residue $j$. Since $\varepsilon_{j}(\lambda)=0$ we then have that $\widetilde{f}_{j} \lambda \in J S(j)$, which by Lemma 5.13 contradicts $n \equiv 0 \bmod p$.

Case 2. $n \not \equiv 0 \bmod p$. In this case $\lambda \in J S(0)$ and $\varphi_{0}(\lambda)=0$ by Lemmas 2.12 and 6.3. From Lemma 5.11 this is equivalent to $\lambda \in J S(0)$ since $n \not \equiv 0 \bmod p$. Notice that

$$
D(\lambda) \otimes M_{1} \cong f_{0} e_{0} D(\lambda) \oplus \sum_{j \geq 1}\left(f_{j} e_{0} D(\lambda)\right)^{\oplus c} \cong D(\lambda) \oplus \sum_{j \geq 1}\left(f_{j} D\left(\widetilde{e}_{0} \lambda\right)\right)^{\oplus c} .
$$

From Lemma 4.2 it follows that

$$
D(\lambda) \otimes D^{(n-1,1)} \cong \sum_{j \geq 1}\left(f_{j} D\left(\widetilde{e}_{0} \lambda\right)\right)^{c} .
$$

From [43, Lemma 3.8] $\widetilde{e}_{0} \lambda \in J S(1)$. Further $\varphi_{0}\left(\widetilde{e}_{0} \lambda\right)=1$. So from Lemma 2.13 there exists $j \geq 1$ with $\varphi_{0}\left(\widetilde{e}_{0} \lambda\right), \varphi_{j}\left(\widetilde{e}_{0} \lambda\right)=1$ and $\varphi_{k}\left(\widetilde{e}_{0} \lambda\right)=0$ for $k \neq 0, j$. If $D(\lambda)$ is of type $\mathrm{M}$ then $D(\lambda) \otimes D^{(n-1,1)} \cong D\left(\widetilde{f}_{j} \widetilde{e}_{0} \lambda\right)$ and $D\left(\widetilde{f}_{j} \widetilde{e}_{0} \lambda\right)$ is of type Q. If $D(\lambda)$ is of type Q then $D(\lambda) \otimes D^{(n-1,1)} \cong D\left(\widetilde{f}_{j} \widetilde{e}_{0} \lambda\right)^{\oplus 2}$ and $D\left(\widetilde{f}_{j} \widetilde{e}_{0} \lambda\right)$ is of type M.

Note that $\widetilde{e}_{0} \lambda=\lambda \backslash A$, since $\lambda$ is $\operatorname{JS}(0)$ and the bottom addable node is always normal. Then $A$ is the bottom addable node of $\widetilde{e}_{0} \lambda$ and it is the conormal node of $\widetilde{e}_{0} \lambda$ of residue 0 . Since $n \geq 4$ and $\lambda$ is $\operatorname{JS}(0)$ we have that $h(\lambda) \geq 2$. If $B$ is the top addable node of $\lambda$ then it is also the top addable node of $\widetilde{e}_{0} \lambda$. Since the top addable node is always conormal, it follows that $\widetilde{f}_{j} \widetilde{e}_{0} \lambda=(\lambda \backslash A) \cup B$. The theorem then follows from Lemma 6.4.

\subsection{Tensor products of basic spin and hooks.}

Theorem 6.6. Let $p \geq 3$. Let $G=\widetilde{\mathrm{S}}_{n}$ or $\widetilde{\mathrm{A}}_{n}$. Assume that $V$ is indexed by an element of $\mathscr{H}_{p}(n)$ and that $W$ is basic spin. If $V$ and $W$ are not 1-dimensional and $V \otimes W$ is irreducible, then one of the following holds:

- $p \neq 5, G=\widetilde{\mathrm{A}}_{5}, V \cong E_{ \pm}^{\left(3,1^{2}\right)}$ and $W \cong E\left(\beta_{5}, \pm\right)$, in which case two of the corresponding tensor products are irreducible and isomorphic to $E((4,1), 0)$, while the other two tensor products are not irreducible.

- $p=3, G=\widetilde{\mathrm{A}}_{6}, V \cong E_{ \pm}^{\left(4,1^{2}\right)}$ and $W \cong E((3,2,1), \pm)$, in which case two of the corresponding tensor products are irreducible and isomorphic to $E((4,2), \pm)$, while the other two tensor products are not irreducible.

In the exceptional cases, if $\chi_{V}$ and $\chi_{W}$ are the characters of $V$ and $W$, we have that $V \otimes W$ is irreducible if and only if $\left(\chi_{V} \chi_{W}\right)(1, \widetilde{2,3,4}, 5)=1$. 
Proof. For $n \leq 12$ the theorem can be proved by looking at decomposition matrices. So we may assume that $n>12$.

We may assume that $k<n / 2$. From [44, Theorem 9.3],

$$
\left[S_{1^{k}} \otimes S((n))\right]=[S((n))]+\sum_{1 \leq j \leq k} d[S((n-j, j))],
$$

where $d=1$ if $n$ is odd and $d=2$ if $n$ is even.

In particular, using Lemmas 2.18 and 2.23 and induction on $k$ if $n \equiv 0 \bmod p$,

- if $n \not \equiv 0 \bmod p$ then

$$
\left[\bar{D}_{k} \otimes D\left(\beta_{n}\right)\right]=\left[D\left(\beta_{n}\right)\right]+\sum_{1 \leq j \leq k} d[S((n-j, j))]
$$

- if $n \equiv 0 \bmod p$ and $k$ is even then

$$
\left[\bar{D}_{k} \otimes D\left(\beta_{n}\right)\right]=\left[D\left(\beta_{n}\right)\right]+\sum_{1 \leq j \leq k / 2}[S((n-2 j, 2 j))]
$$

- if $n \equiv 0 \bmod p$ and $k$ is odd then

$$
\left[\bar{D}_{k} \otimes D\left(\beta_{n}\right)\right]=\sum_{0 \leq j \leq(k-1) / 2}[S((n-2 j-1,2 j+1))] .
$$

When $n \equiv 0 \bmod p$ then $D\left(\beta_{n}\right)$ is a composition factor of $S((n-1,1))$ by 45 , Table IV]. So $D\left(\beta_{n}\right)$ is always a composition factor of $\bar{D}_{k} \otimes D\left(\beta_{n}\right)$ (as supermodule). Since $\bar{D}_{k} \otimes D\left(\beta_{n}, \delta\right)$ is irreducible if and only if $\bar{D}_{k} \otimes D\left(\beta_{n},-\delta\right)$ is irreducible and since $\bar{D}_{k}$ is not 1-dimensional, it follows that $\bar{D}_{k} \otimes D\left(\beta_{n}, \delta\right)$ is not irreducible. Similarly if $k \neq(n-c) / 2$ then $\bar{E}_{k} \otimes E\left(\beta_{n}, \delta^{\prime}\right)$ is not irreducible.

So assume now that $k=(n-c) / 2$. Note that in this case either $n$ is odd with $n \not \equiv 0 \bmod p$ or $n$ is even with $n \equiv 0 \bmod p$, so $D\left(\beta_{n}\right)$ is of type $\mathrm{M}$ and then $\delta^{\prime}= \pm$. By Lemmas 2.18 and 2.23 we have

$$
\operatorname{dim}\left(\left(\bar{E}_{k}\right)_{ \pm} \otimes E\left(\beta_{n}, \pm\right)\right)=\frac{1}{2}\left(\begin{array}{c}
n-c \\
(n-c) / 2
\end{array}\right) 2^{(n-c-2) / 2}=2^{(n-c-4) / 2}\left(\begin{array}{c}
n-c \\
(n-c) / 2
\end{array}\right) .
$$

Let $d_{j}$ be the dimension of any simple spin module of $\widetilde{\mathrm{A}}_{n}$ indexed by $(n-j, j)$ in characteristic 0 . For $1 \leq j \leq k$ we have

$$
d_{j}=\frac{1}{2} \operatorname{dim} S((n-j, j))=2^{(n-c-2) / 2} \frac{n-2 j}{n-j}\left(\begin{array}{c}
n-1 \\
j
\end{array}\right) .
$$

Note that if $\left(\bar{E}_{k}\right)_{ \pm} \otimes E\left(\beta_{n}, \pm\right)$ is irreducible then it is not isomorphic to $E\left(\beta_{n}, \pm\right)$ (since $\left(\bar{E}_{k}\right)_{ \pm}$is not 1-dimensional). In order to prove that $\left(\bar{E}_{k}\right)_{ \pm} \otimes E\left(\beta_{n}, \pm\right)$ is not irreducible it is then enough to prove that $\operatorname{dim}\left(\left(E_{k}\right)_{ \pm} \otimes E\left(\beta_{n}, \pm\right)\right)>d_{j}$ for any $1 \leq j \leq k$. If $n$ is even note that

$$
2\left(\begin{array}{c}
n-2 \\
(n-2) / 2
\end{array}\right)=\frac{n}{n-1}\left(\begin{array}{c}
n-1 \\
(n-2) / 2
\end{array}\right)>\left(\begin{array}{c}
n-1 \\
(n-2) / 2
\end{array}\right) .
$$

So it is enough to prove that

$$
\left(\begin{array}{c}
n-1 \\
\lfloor(n-1) / 2\rfloor
\end{array}\right)=\left(\begin{array}{c}
n-1 \\
(n-c) / 2
\end{array}\right)>2^{c} \frac{n-2 j}{n-j}\left(\begin{array}{c}
n-1 \\
j
\end{array}\right)
$$

for any $1 \leq j \leq k=\lfloor(n-1) / 2\rfloor$. 
If $j>3 / 7 n$ then $4(n-2 j) /(n-j)<1$ and so the above inequality clearly holds. So we may assume that $j \leq 3 / 7 n$. In this case it is enough to prove that

$$
\frac{\left(\begin{array}{c}
n-1 \\
(n-1) / 2\rfloor
\end{array}\right)}{\left(\begin{array}{c}
n-1 \\
j
\end{array}\right)}=\prod_{i=j+1}^{\lfloor(n-1) / 2\rfloor} \frac{n-i}{i}>4 .
$$

It is enough to prove this for $j=\lfloor 3 / 7 n\rfloor$. If $n \geq 152$ then

$$
\prod_{i=\lfloor 3 / 7 n\rfloor+1}^{\lfloor(n-1) / 2\rfloor} \frac{n-i}{i} \geq \prod_{a=1}^{\lfloor(n-1) / 2\rfloor-\lfloor 3 / 7 n\rfloor} \frac{4 / 7 n-a}{3 / 7 n+a} \geq \prod_{a=1}^{9} \frac{4 / 7 n-a}{3 / 7 n+a}>4 .
$$

Using the above formulas, it can be checked that for $n \leq 151$ and $n \leq 3 / 7 n$ we have $\operatorname{dim}\left(\left(\bar{E}_{n, k}\right)_{ \pm} \otimes E\left(\beta_{n}, \pm\right)\right)>d_{j}$, unless possibly if $n \leq 20$ is even with $n \equiv 0 \bmod p$. In these cases notice that it is enough to prove that $\operatorname{dim}\left(\left(\bar{E}_{n, k}\right)_{ \pm} \otimes E\left(\beta_{n}, \pm\right)\right)>d_{j}$ for $j$ odd if $n \equiv 0 \bmod 4$ or for $j$ even if $n \equiv 2 \bmod 4$, which again can be checked using the above formulas since we are assuming $n>12$.

\subsection{Tensor products of basic spin and two row partitions.}

Theorem 6.7. Let $p \geq 3$ and $G \in\left\{\widetilde{\mathrm{S}}_{n}, \widetilde{\mathrm{A}}_{n}\right\}$. Let $V$ be a simple non-spin module indexed by $\lambda \in \mathscr{P}_{p}(n)$ with $\min \left\{h(\lambda), h\left(\lambda^{\mathrm{M}}\right)\right\}=2$ and $W$ be basic spin. If $V \otimes W$ is irreducible then $\lambda$ is $J S$ and $n \not \equiv 0, \pm 2 \bmod p$. Further in this case:

- if $G=\widetilde{\mathrm{S}}_{n}$ and $n$ is even then $V \otimes W \cong D(\mu, 0)$ is irreducible with $\mu=$ $\beta_{\lambda_{1}}+\beta_{\lambda_{2}}$ if $\lambda_{1} \neq \lambda_{2}$ or $\mu=\beta_{n / 2+1} \cup \beta_{n / 2-1}$ if $\lambda_{1}=\lambda_{2}$,

- if $G=\widetilde{\mathrm{S}}_{n}$ and $n$ is odd then $V \otimes W$ is not irreducible,

- if $G=\widetilde{\mathrm{A}}_{n}$ and $n$ is even then $V \otimes W$ is not irreducible,

- if $G=\widetilde{\mathrm{A}}_{n}$ and $n$ is odd then $V \otimes W \cong E(\mu, 0)$ is irreducible with $\mu=$ $\beta_{\lambda_{1}}+\beta_{\lambda_{2}}$ if $\lambda_{1} \neq \lambda_{2}+p-2$ or $\mu=\beta_{\lambda_{1}} \cup \beta_{\lambda_{2}}$ if $\lambda=\lambda_{2}+p-2$.

Proof. For $n \leq 9$ the theorem can be proved looking at decomposition matrices. So assume that $n \geq 10$. Note that $V \cong D^{\lambda} \downarrow_{G}$ by [33, Lemma 1.8]. Further we may assume that $h(\lambda)=2$. In view of Theorem 6.5 we may also assume that $\lambda_{2} \geq 2$ (since $(n-1,1)$ is JS if and only if $n \equiv 0 \bmod p)$. Note that in this case $\lambda \notin \mathscr{H}_{p}(n)$ (the case $p=3$ and $\lambda=(n)^{\mathrm{M}}$ is excluded by assumption). It is easy to see that $\lambda$ is JS if and only if $\lambda_{1}=\lambda_{2}$ or $\lambda_{1}-\lambda_{2} \equiv-2 \bmod p$.

Let $W^{\prime}=D\left(\beta_{n}\right)$ or $E\left(\beta_{n}\right)$ (depending on $G$ ). Further from Lemmas 5.20 and 5.21 we have that $\bar{D}_{0} \oplus \bar{D}_{2} \subseteq \operatorname{End}_{F}(W)$ and $\bar{D}_{0} \oplus \bar{D}_{1} \oplus \bar{D}_{2} \oplus \bar{D}_{3} \subseteq \operatorname{Hom}_{F}\left(W^{\prime}, W\right)$.

If $\lambda$ is not JS then we have that $\bar{D}_{0} \oplus \bar{D}_{2}$ or $\bar{D}_{0} \oplus \bar{D}_{1} \oplus \bar{D}_{3}$ is contained in $\operatorname{End}_{F}(V)$ from Lemmas 5.2, 5.3 and 5.4. It follows that

$$
\operatorname{dim} \operatorname{Hom}_{G}\left(\operatorname{End}_{F}(V), \operatorname{End}_{F}(W)\right) \geq 2
$$

or

$$
\operatorname{dim} \operatorname{Hom}_{G}\left(\operatorname{End}_{F}(V), \operatorname{Hom}_{F}\left(W^{\prime}, W\right)\right) \geq 3 .
$$

So $V \otimes W$ is not irreducible (in the second case by Lemma 6.1).

So assume now that $\lambda$ is JS. In view of Lemmas 2.23 and 3.11 we have that $\bar{D}_{0} \oplus \bar{D}_{2}$ or $\bar{D}_{0} \oplus \bar{D}_{3}$ is contained in $\operatorname{End}_{F}(V)$. So

$$
\operatorname{dim} \operatorname{Hom}_{G}\left(\operatorname{End}_{F}(V), \operatorname{Hom}_{F}\left(W^{\prime}, W\right)\right) \geq 2 .
$$


So by Lemmas 6.1 and 6.2 if $G=\widetilde{\mathrm{S}}_{n}$ then $D^{\lambda} \otimes D\left(\beta_{n}, \delta\right)$ is irreducible if and only if $D\left(\beta_{n}\right)$ is of type $\mathrm{Q}, D^{\lambda} \otimes D\left(\beta_{n}\right)$ has a composition factor of type $\mathrm{M}$ and

$$
\operatorname{dim} \operatorname{Hom}_{\widetilde{\mathrm{S}}_{n}}\left(\operatorname{End}_{F}\left(D^{\lambda}\right), \operatorname{Hom}_{F}\left(D\left(\beta_{n}\right), D\left(\beta_{n}, \delta\right)\right)\right)=2 .
$$

Similarly if $G=\widetilde{\mathrm{A}}_{n}$ then $E^{\lambda} \otimes E\left(\beta_{n}, \delta^{\prime}\right)$ is irreducible if and only if $D\left(\beta_{n}\right)$ is of type $\mathrm{M}, D^{\lambda} \otimes D\left(\beta_{n}\right)$ has a composition factor of type $\mathrm{Q}$ and

$$
\operatorname{dim} \operatorname{Hom}_{\widetilde{\mathrm{A}}_{n}}\left(\operatorname{End}_{F}\left(E^{\lambda}\right), \operatorname{Hom}_{F}\left(E\left(\beta_{n}\right), E\left(\beta_{n}, \delta^{\prime}\right)\right)\right)=2 .
$$

On the other hand if $D^{\lambda} \otimes D\left(\beta_{n}\right)$ has a composition factor of the same type as $D\left(\beta_{n}\right)$ then $V \otimes W$ is not irreducible.

Note that $\left[D^{\lambda}\right]=\left[S^{\lambda}\right]+\sum_{j<\lambda_{2}} d_{j}\left[S^{(n-j, j)}\right]$ for some $d_{j} \in \mathbb{Z}$ with $d_{j} \neq 0$ only if $D^{\lambda}$ and $D^{(n-j, j)}$ are in the same block by [22, Corollary 12.2]. Further $\left[D\left(\beta_{n}\right)\right]=$ $d[S((n))]$ for some $d>0$ by Lemma 2.18.

If $\lambda_{1}=\lambda_{2}$ then $D^{\lambda}$ and $D^{\left(\lambda_{1}+1, \lambda_{1}-1\right)}$ are in different blocks and so by 44 , Theorem 9.3]

$$
\left[D^{\lambda} \otimes D\left(\beta_{n}\right)\right]=c\left[S\left(\left(\lambda_{1}+1, \lambda_{1}-1\right)\right)\right]+\sum_{j<\lambda_{1}-1} c_{j}[S((n-j, j))]
$$

for some $c, c_{j} \in \mathbb{Q}$ with $c>0$. In this case let $\nu:=(n / 2+1, n / 2-1)=\left(\lambda_{1}+1, \lambda_{2}-1\right)$.

If $\lambda_{1}>\lambda_{2}$ then

$$
\left[D^{\lambda} \otimes D\left(\beta_{n}\right)\right]=c[S(\lambda)]+\sum_{j<\lambda_{2}} c_{j}[S((n-j, j))]
$$

for some $c, c_{j} \in \mathbb{Q}$ with $c>0$. In this case let $\nu:=\lambda$. Note that $\lambda_{1} \geq \lambda_{2}+p-2$. Further if $p=3$ then by assumption $\lambda_{1}-\lambda_{2} \geq 4$.

From [39, Theorems 1.2,1.3] there exists a composition factor $D(\mu)$ of $S(\nu)$ which is not a composition factor of $S\left(\left(\pi_{1}, \pi_{2}\right)\right)$ for $\left(\pi_{1}, \pi_{2}\right) \in \mathscr{R} \mathscr{P}_{0}(n)$ with $\pi_{1}>\nu_{1}$. Then $D(\mu)$ is a composition factor of $D^{\lambda} \otimes D\left(\beta_{n}\right)$.

Case 1. $n \equiv 0 \bmod p$. In this case any composition factor of $S((n-j, j))$ with $j<n / 2$ is in the same block as $D\left(\beta_{n}\right)$, so they have the same type and then $V \otimes W$ is not irreducible in this case.

Case 2. $n \equiv \pm 2 \bmod p$. In this case it can be checked that if $\lambda_{1}=\lambda_{2}$ then one part of $(n / 2+1, n / 2-1)$ is divisible by $p$, while if $\lambda_{1}>\lambda_{2}$ then one part of $\lambda$ is divisible by $p$ (since in this case $\lambda_{1}-\lambda_{2} \equiv p-2 \bmod p$ ). So $S(\nu)$ is in the same block as $S((n))$ and then again $V \otimes W$ is not irreducible.

Case 3. $n \not \equiv 0, \pm 2 \bmod p$. In this case $p \geq 5$ and so Lemmas 2.23, 2.24, 5.5 and 5.20

$$
\begin{aligned}
& \operatorname{dim} \operatorname{Hom}_{\widetilde{\mathrm{S}}_{n}}\left(\operatorname{End}_{F}\left(D^{\lambda}\right), \operatorname{Hom}_{F}\left(D\left(\beta_{n}\right), D\left(\beta_{n}, \delta\right)\right)\right)=2, \\
& \operatorname{dim} \operatorname{Hom}_{\widetilde{\mathrm{A}}_{n}}\left(\operatorname{End}_{F}\left(E^{\lambda}\right), \operatorname{Hom}_{F}\left(E\left(\beta_{n}\right), E\left(\beta_{n}, \delta^{\prime}\right)\right)\right)=2 .
\end{aligned}
$$

Further if $\lambda_{1}=\lambda_{2}$ then $p \nmid n / 2 \pm 1$, while if $\lambda_{1}>\lambda_{2}$ then $p \nmid \lambda_{1}, \lambda_{2}$. Since $n \not \equiv 0 \bmod p$ it can then be easily checked that $D(\mu)$ and $D\left(\beta_{n}\right)$ are of different type. So $D^{\lambda} \otimes D\left(\beta_{n}, \delta\right)$ is irreducible if and only if $n$ is even and in this case $D^{\lambda} \otimes D\left(\beta_{n}, \delta\right) \cong D(\mu, 0)$. Similarly $E^{\lambda} \otimes E\left(\beta_{n}, \delta^{\prime}\right)$ is irreducible if and only if $n$ is even and in this case $E^{\lambda} \otimes E\left(\beta_{n}, \delta^{\prime}\right) \cong E(\mu, 0)$. The theorem then follows from [39, Theorems 1.2, 1.3] to identify $\mu$. 


\subsection{Tensor products of basic spin and three row partitions.}

Theorem 6.8. Let $p=3$ and $G \in\left\{\widetilde{\mathrm{S}}_{n}, \widetilde{\mathrm{A}}_{n}\right\}$. Let $\lambda \in \mathscr{P}_{p}(n) \backslash \mathscr{H}_{3}(p)$ with $\min \left\{h(\lambda), h\left(\lambda^{\mathrm{M}}\right)\right\}=3, V$ be a simple non-spin module indexed by $\lambda$ and $W$ be basic spin. Then $V \otimes W$ is not irreducible.

Proof. We may assume that $h(\lambda)=3$. Since $\lambda \notin \mathscr{H}_{3}(n)$ we then have that $\lambda=$ $\left(\lambda_{1}, \lambda_{2}, \lambda_{3}\right)$ with $\lambda_{1} \geq \lambda_{2}+2, \lambda_{2} \geq \lambda_{3}+2$ and $\lambda_{3} \geq 1$. In particular $n \geq 9$. Further it is easy to check that $\lambda \neq \lambda^{\mathrm{M}}$, so $V \cong D^{\lambda} \downarrow_{G}$.

If $W^{\prime}=D\left(\beta_{n}\right)$ if $G=\widetilde{\mathrm{S}}_{n}$ or $W^{\prime}=D\left(\beta_{n}\right)$ if $G=\widetilde{\mathrm{A}}_{n}$ then

$$
\bar{D}_{0} \oplus \bar{D}_{1} \oplus \bar{D}_{2} \oplus \bar{D}_{3} \subseteq \operatorname{Hom}_{F}\left(W^{\prime}, W\right)
$$

by Lemma 5.20. If $\lambda$ is not JS then

$$
\bar{D}_{0} \oplus \bar{D}_{1} \oplus \bar{D}_{k} \subseteq \operatorname{End}_{F}(V)
$$

with $2 \leq k \leq 3$ from Lemmas 5.2 and 5.6 . So in this case $V \otimes W$ is not irreducible by Lemma 6.1

So we may assume that $\lambda$ is JS. So $\lambda_{1}-\lambda_{2}, \lambda_{2}-\lambda_{3} \equiv 1 \bmod 3$ and then we have $\lambda_{1} \geq \lambda_{2}+4$ and $\lambda_{2} \geq \lambda_{3}+4$. From Lemmas 2.23 and 3.11 we have that $\bar{D}_{2}$ or $\bar{D}_{3}$ is contained in $\operatorname{End}_{F}(V)$. Since we always have $\bar{D}_{0} \subseteq \operatorname{End}_{F}(V)$ from Lemmas 6.1 and 6.2 to prove that $V \otimes W$ is not irreducible it is enough to prove that $D^{\lambda} \otimes D\left(\beta_{n}\right)$ has a composition factor of the same type as $D\left(\beta_{n}\right)$. Note that by Lemma 2.18 and [44, Theorem 9.3] we have that

$$
\left[D^{\lambda} \otimes D\left(\beta_{n}\right)\right]=c[S(\lambda)]+\sum_{\mu \in \mathscr{R} \mathscr{P}_{0}(n): \mu \triangleright \lambda} c_{\mu}[S(\mu)]
$$

with $c>0$. From Lemmas 2.17 and 2.19] we then have that if $\nu=\lambda^{R}=\beta_{\lambda_{1}}+$ $\beta_{\lambda_{2}}+\beta_{\lambda_{3}}$ then $D(\nu)$ is a composition factor of $D^{\lambda} \otimes D\left(\beta_{n}\right)$ (since $\lambda_{1} \geq \lambda_{2}+4$ and $\left.\lambda_{2} \geq \lambda_{3}+4\right)$. From $\lambda_{1}-\lambda_{2}, \lambda_{2}-\lambda_{3} \equiv 1 \bmod 3$ we have that $n \equiv 0 \bmod 3$ and one of $\lambda_{1}, \lambda_{2}$ and $\lambda_{3}$ is divisible by 3. In particular $S(\lambda)$ and $S((n))$ are in the same block and so $D(\nu)$ and $D\left(\beta_{n}\right)$ are of the same type. So $V \otimes W$ is not irreducible.

6.5. Tensor products of basic and second basic spin. The following result will only be needed for $p=3$, but the proof in the general case is the same as the proof for the case $p=3$ only, so we present it here in the general version. By definition, for $G=\widetilde{\mathrm{S}}_{n}$ or $\widetilde{\mathrm{A}}_{n}$, second basic spin modules of $G$ are composition factors of the reduction modulo $p$ of $S((n-1,1)) \downarrow_{G}$ which are not basic spin modules.

Theorem 6.9. Let $p \geq 3, n \geq 6$ and $G=\widetilde{\mathrm{S}}_{n}$ or $\widetilde{\mathrm{A}}_{n}$. Assume that $V$ is second basic spin and that $W$ is basic spin. Then $V \otimes W$ is not irreducible.

Proof. From Lemma 2.18 and [45, Table IV] we have that any composition factor of $V \otimes W$ is a composition factor of the reduction modulo $p$ of $S((n-1,1)) \otimes S((n))$. So from [44, Theorem 9.3], any composition factor of $V \otimes W$ is a composition factor of a Specht module of the form $S^{\left(n-k, 1^{k}\right)}$ with $0 \leq k \leq n-1$ or $S^{\left(n-k, 2,1^{k-2}\right)}$ with $2 \leq k \leq n-2$. Notice also that by [45, Tables III and IV]

$$
\operatorname{dim} V \otimes W \geq 2^{n-4}(n-4) .
$$

It can be computed that

$$
\operatorname{dim} S^{\left(n-k, 1^{k}\right)}=\left(\begin{array}{c}
n-1 \\
k
\end{array}\right), \quad \operatorname{dim} S^{\left(n-k, 2,1^{k-2}\right)}=\left(\begin{array}{l}
n \\
k
\end{array}\right) \frac{(n-k-1)(k-1)}{n-1} .
$$


Since

$$
\frac{(n-k-1)(k-1)}{n-1} \leq \frac{(n-2)^{2}}{4(n-1)} \leq \frac{n-2}{4},
$$

it is enough to prove that

$$
\left(\begin{array}{c}
n \\
\lfloor n / 2\rfloor
\end{array}\right) \frac{n-2}{4}<2^{n-4}(n-4)
$$

that is

$$
\frac{\left(\begin{array}{c}
n \\
\lfloor n / 2\rfloor
\end{array}\right)(n-2)}{2^{n-2}(n-4)}<1 .
$$

Notice that $(n-2) /(n-4)$ is decreasing as is $\left(\begin{array}{c}n \\ \lfloor n / 2\rfloor\end{array}\right) / 2^{n-2}$, since

$$
\begin{aligned}
\left(\begin{array}{c}
n \\
\lfloor n / 2\rfloor
\end{array}\right) / 2^{n-2} & =\left(\begin{array}{c}
n-1 \\
\lfloor n / 2\rfloor
\end{array}\right) / 2^{n-2}+\left(\begin{array}{c}
n-1 \\
\lfloor n / 2\rfloor-1
\end{array}\right) / 2^{n-2} \\
& \leq\left(\begin{array}{c}
n-1 \\
\lfloor(n-1) / 2\rfloor
\end{array}\right) / 2^{(n-1)-2} .
\end{aligned}
$$

Since $\left(\begin{array}{c}15 \\ 7\end{array}\right) \cdot 13 /\left(2^{13} \cdot 11\right)<1$, the lemma holds for $n \geq 15$.

For $6 \leq n \leq 14$ the lemma can be checked by looking at decomposition matrices for $S_{n}$ and $A_{n}$ to find the dimension of composition factors of the reduction modulo $p$ of the modules $S^{\left(n-k, 1^{k}\right)}$ and $S^{\left(n-k, 2,1^{k-2}\right)}$ as well as exact formulas for $\operatorname{dim} V \otimes W$ coming [45, Tables III and IV].

\section{Proofs of Theorems 1.1 and 1.2}

By [6, 7, 37, 38, 46] we may assume that $W$ is a spin representation. Further we may assume that neither $V$ nor $W$ is 1-dimensional. For $n \leq 12$ the theorems can be proved using GAP [18] or looking at decomposition matrices (and using Lemma 2.17 to identify modular spin representations). So assume that $n \geq 13$. It can then be checked (using Lemma 2.24 and [3, Lemma 2.2] to help check some cases) that if $\alpha$ is one of $(n-3,3),\left(n-3,1^{3}\right),\left(n-5,1^{5}\right)$ or $\left(n-5,3,1^{2}\right)$ (the last one only for $p=3$ ) and $\alpha \in \mathscr{P}_{p}(n)$, then $\alpha>\alpha^{\mathrm{M}}$. Let $G \in\left\{\widetilde{\mathrm{S}}_{n}, \widetilde{\mathrm{A}}_{n}\right\}$ depending on which theorem we are considering. Since $n \geq 13$ we have from [33. Lemma 1.8] that $(n-k, k) \neq(n-k, k)^{\mathrm{M}}$ for any $0 \leq k \leq n / 2$. Further the modules $\bar{E}_{k}$ for $0 \leq k \leq 5$ are defined and they are simple and pairwise non-isomorphic.

Case 1. $p \geq 5$ and neither $V$ nor $W$ is a basic spin representation or a natural representation (a non-spin representation indexed by $(n-1,1)$ or $\left.(n-1,1)^{\mathrm{M}}\right)$.

Parts of this case could be proved using results from [11,34. However the cases where $V$ is a non-spin representation which is indexed by a 2-row JS partition (or its Mullineux dual) or if $G=\widetilde{\mathrm{A}}_{n}$ and $V$ is non spin and indexed by a Mullineux-fixed partition are not covered by results from [11,34.

By Lemmas 3.8 and 3.12 there exist $\varphi_{3} \in \operatorname{Hom}_{G}\left(M_{3}, \operatorname{End}_{F}(W)\right)$ and $\varphi_{1^{3}} \in$ $\operatorname{Hom}_{G}\left(M_{1^{3}}, \operatorname{End}_{F}(W)\right)$ which do not vanish on $S_{3}$ and $S_{1^{3}}$ respectively. Further from Lemmas 3.7 3.8 and 3.11 we have that there exists $\psi_{3} \in \operatorname{Hom}_{G}\left(M_{3}, \operatorname{End}_{F}(V)\right.$ ) or $\psi_{1^{3}} \in \operatorname{Hom}_{G}\left(M_{1^{3}}, \operatorname{End}_{F}(V)\right)$ which does not vanish on $S_{3}$ or $S_{1^{3}}$. Since $M_{0}=S_{0}$ is the trivial module, so there also always exist non-zero $\varphi_{0} \in \operatorname{Hom}_{G}\left(M_{0}, \operatorname{End}_{F}(W)\right)$ and $\psi_{0} \in \operatorname{Hom}_{G}\left(M_{0}, \operatorname{End}_{F}(V)\right)$, we then have from Lemma 2.22 that

$$
\operatorname{dim} \operatorname{End}_{G}(V \otimes W)=\operatorname{dim} \operatorname{Hom}_{G}\left(\operatorname{End}_{F}(V), \operatorname{End}_{F}(W)\right) \geq 2
$$

and so $V \otimes W$ is not irreducible. 
Case 2. $p=3$, neither $V$ nor $W$ is a basic spin representation or a natural representation and either $n \equiv 2 \bmod 3$ and $V$ is not a non-spin representation indexed by $(n-2,2)$ or $(n-2,2)^{\mathrm{M}}$ or $n \not \equiv 2 \bmod 3$.

This case holds similarly to the previous case, using Lemmas 3.7, 3.8, 3.9, 3.10 and 5.1 (so using $M_{3,1^{2}}$ instead of $M_{1^{3}}$ ).

Case 3. $p=3, n \equiv 2 \bmod 3$ and $V$ is a non-spin representation indexed by $(n-2,2)$ or $(n-2,2)^{\mathrm{M}}$ and $W$ is not basic spin.

We have that $(n-2,2) \neq(n-2,2)^{\mathrm{M}}$. So (up to tensoring with $\left.\operatorname{sgn}\right) V \cong$ $D^{(n-2,2)}$ or $E^{(n-2,2)}$. From [11, Corollary 3.9] and Lemma 4.1 there exists $\psi_{2} \in$ $\operatorname{Hom}_{G}\left(M_{2}, \operatorname{End}_{F}(V)\right)$ which does not vanish on $S_{2}$. From [43, Lemma 3.7] we have that, for $p=3$, any $\operatorname{JS}(0)$ partition in $\mathscr{R} \mathscr{P}_{3}(m)$ is of the form $\beta_{\mu_{1}}+\ldots+\beta_{\mu_{k}}$ with $\mu_{j} \equiv 0 \bmod 3$ for $j<k$ and $\mu_{k}=1$ or $\mu_{k} \equiv 0 \bmod 3$. Since $n \equiv 2 \bmod 3$ there is then no $\mathrm{JS}(0)$ partition in $\mathscr{R}_{3}(n)$. Let $\nu$ be the partition indexing $W$. We will now consider $G=\widetilde{\mathrm{S}}_{n}$, the case $G=\widetilde{\mathrm{A}}_{n}$ being similar. By Lemma 5.19 there exists $\varphi_{2} \in \operatorname{Hom}_{\widetilde{\mathrm{S}}_{n}}\left(M_{2}, \operatorname{End}_{F}(W)\right)$ which does not vanish on $S_{2}$ or $W \cong D(\nu, \pm)$ and there exist $\varphi_{2}^{\prime}, \varphi_{2}^{\prime \prime} \in \operatorname{Hom}_{\widetilde{\mathrm{S}}_{n}}\left(M_{2}, \operatorname{Hom}_{F}(D(\lambda, \pm), D(\lambda, \mp))\right)$ which are linearly independent over $S_{2}$. In the first case we can conclude as in Case 1. In the second case we have by Lemma 2.22 that

$$
\begin{aligned}
& \operatorname{dim} \operatorname{Hom}_{\widetilde{\mathrm{S}}_{n}}(V \otimes D(\lambda, \pm), V \otimes D(\lambda, \mp)) \\
& =\operatorname{dim} \operatorname{Hom}_{\widetilde{\mathrm{S}}_{n}}\left(\operatorname{End}_{F}(V), \operatorname{Hom}_{F}(D(\lambda, \pm), D(\lambda, \mp))\right) \\
& \geq 2 .
\end{aligned}
$$

Since $D(\lambda,+)$ and $D(\lambda,-)$ have the same dimension, this contradicts $V \otimes W$ being irreducible.

Case $4 . V$ is a natural module.

Up to tensoring with sgn we have that $V \cong D^{(n-1,1)}$ or $E^{(n-1,1)}$. The theorems then follow from Theorem 6.5 .

Case 5. $V$ and $W$ are basic spin.

Let $A:=D\left(\beta_{n}\right)$ if $G=\widetilde{\mathrm{S}}_{n}$ or $A:=E\left(\beta_{n}\right)$ if $G=\widetilde{\mathrm{A}}_{n}$. Then by Lemma 5.20 $\operatorname{dim} \operatorname{Hom}_{G}(A \otimes A, V \otimes W)=\operatorname{dim} \operatorname{Hom}_{G}\left(\operatorname{Hom}_{F}(V, A) \operatorname{Hom}_{F}(A, W) \geq 5\right.$.

Since $\operatorname{dim} A \leq 2 \operatorname{dim} V$ and $\operatorname{dim} V=\operatorname{dim} W$, it follows that $V \otimes W$ is not irreducible.

Case 6. $W$ is basic spin and $V$ is either a non-spin representation indexed by $\lambda \notin \mathscr{H}_{p}(n)$ with $h(\lambda), h\left(\lambda^{\mathrm{M}}\right) \geq 3+\delta_{p=3}$ or a spin representation indexed by $\mu \neq \beta_{n}$ with $\mu_{1} \geq 5$.

From Lemmas 2.23 and 2.24 we have that $\left[S_{1^{3}}\right]=\left[\bar{D}_{3}\right]+\delta_{p \mid n}\left[\bar{D}_{2}\right]$ and $\left[S_{1^{5}}\right]=$ $\left[\bar{D}_{5}\right]+\delta_{p \mid n}\left[\bar{D}_{4}\right]$.

From Lemmas 3.11 and 3.12 there exists $0 \neq \varphi_{3} \in \operatorname{Hom}_{G}\left(S_{1^{3}}, \operatorname{End}_{F}(V)\right)$ and from Lemmas 3.13 and 3.14 there exists $0 \neq \varphi_{5} \in \operatorname{Hom}_{G}\left(S_{1^{5}}, \operatorname{End}_{F}(V)\right)$. In par-

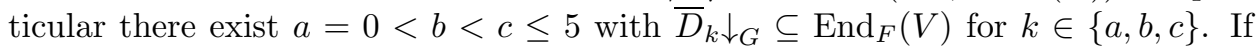
again $A:=D\left(\beta_{n}\right)$ if $G=\widetilde{\mathrm{S}}_{n}$ or $A:=E\left(\beta_{n}\right)$ if $G=\widetilde{\mathrm{A}}_{n}$ then by Lemma 5.20

$$
\operatorname{dim} \operatorname{Hom}_{G}(V \otimes A, V \otimes W)=\operatorname{dim} \operatorname{Hom}_{G}\left(\operatorname{End}_{F}(V), \operatorname{Hom}_{F}(A, W) \geq 3\right.
$$

and so $V \otimes W$ is not irreducible by Lemma 6.1 .

Case 7. $W$ is basic spin and $V$ is a non-spin representation indexed by $\lambda \in \mathscr{H}_{p}(n)$.

In this case the theorems hold by Theorem 6.6. 
Case 8. $W$ is basic spin and $V$ is a non-spin representation indexed by $\lambda$ with $h(\lambda), h\left(\lambda^{\mathrm{M}}\right)=2$.

This case is covered by Theorem 6.7.

Case 9. $p=3, W$ is basic spin and $V$ is a non-spin representation indexed by $\lambda \notin \mathscr{H}_{3}(n)$ with $h(\lambda), h\left(\lambda^{\mathrm{M}}\right)=3$.

In this case $V \otimes W$ is not irreducible by Theorem 6.8

Case 10. $W$ is basic spin and $V$ is a spin representation indexed by $\mu \neq \beta_{n}$ with $\mu_{1} \leq 4$

Note that in this case $p=3$ since $n \geq 13$. Since $\mu \neq \beta_{n}$ we have that $\mu=$ $\left(4, \beta_{n-4}\right)=\beta_{n-1}+\beta_{1}$. In view of Lemma 2.19 and [45, Table IV] we have that $V$ is second basic spin. So the theorems hold by Theorem 6.9.

\section{ACKNOWLEDGMEnTs}

The author thanks Alexander Kleshchev for some comments on the paper. The author also thanks the referees for comments. The author would like to thank the Isaac Newton Institute for Mathematical Sciences for support and hospitality during the programme "Groups, representations and applications: new perspectives" when work on this paper was undertaken.

\section{REFERENCES}

[1] M. Aschbacher, On the maximal subgroups of the finite classical groups, Invent. Math. 76 (1984), no. 3, 469-514, DOI 10.1007/BF01388470. MR746539

[2] M. Aschbacher and L. Scott, Maximal subgroups of finite groups, J. Algebra 92 (1985), no. 1, 44-80, DOI 10.1016/0021-8693(85)90145-0. MR772471

[3] A. A. Baranov, A. S. Kleshchev, and A. E. Zalesskii, Asymptotic results on modular representations of symmetric groups and almost simple modular group algebras, J. Algebra 219 (1999), no. 2, 506-530, DOI 10.1006/jabr.1999.7923. MR1706817

[4] Christine Bessenrodt, On mixed products of complex characters of the double covers of the symmetric groups, Pacific J. Math. 199 (2001), no. 2, 257-268, DOI 10.2140/pjm.2001.199.257. MR.1847134

[5] C. Bessenrodt and A. Kleshchev, On Kronecker products of complex representations of the symmetric and alternating groups, Pacific J. Math. 190 (1999), no. 2, 201-223, DOI 10.2140/pjm.1999.190.201. MR.1722888

[6] Christine Bessenrodt and Alexander S. Kleshchev, On tensor products of modular representations of symmetric groups, Bull. London Math. Soc. 32 (2000), no. 3, 292-296, DOI 10.1112/S0024609300007098. MR.1750169

[7] Christine Bessenrodt and Alexander S. Kleshchev, Irreducible tensor products over alternating groups, J. Algebra 228 (2000), no. 2, 536-550, DOI 10.1006/jabr.2000.8284. MR.1764578

[8] Christine Bessenrodt and Alexander S. Kleshchev, On Kronecker products of spin characters of the double covers of the symmetric groups, Pacific J. Math. 198 (2001), no. 2, 295-305, DOI 10.2140/pjm.2001.198.295. MR.1835510

[9] C. Bessenrodt and J. B. Olsson, On residue symbols and the Mullineux conjecture, J. Algebraic Combin. 7 (1998), no. 3, 227-251, DOI 10.1023/A:1008618621557. MR1616083

[10] Jonathan Brundan and Alexander Kleshchev, On translation functors for general linear and symmetric groups, Proc. London Math. Soc. (3) 80 (2000), no. 1, 75-106, DOI 10.1112/S0024611500012132. MR 1719176

[11] Jonathan Brundan and Alexander S. Kleshchev, Representations of the symmetric group which are irreducible over subgroups, J. Reine Angew. Math. 530 (2001), 145-190, DOI 10.1515/crll.2001.002. MR 1807270

[12] Jonathan Brundan and Alexander Kleshchev, Hecke-Clifford superalgebras, crystals of type $A_{2 l}^{(2)}$ and modular branching rules for $\hat{S}_{n}$, Represent. Theory 5 (2001), 317-403, DOI 10.1090/S1088-4165-01-00123-6. MR.1870595 
[13] Jonathan Brundan and Alexander Kleshchev, Projective representations of symmetric groups via Sergeev duality, Math. Z. 239 (2002), no. 1, 27-68, DOI 10.1007/s002090100282. MR.1879328

[14] Jonathan Brundan and Alexander Kleshchev, Modular representations of the supergroup $Q(n)$. I, J. Algebra 260 (2003), no. 1, 64-98, DOI 10.1016/S0021-8693(02)00620-8. Special issue celebrating the 80th birthday of Robert Steinberg. MR.1973576

[15] Jonathan Brundan and Alexander Kleshchev, James' regularization theorem for double covers of symmetric groups, J. Algebra 306 (2006), no. 1, 128-137, DOI 10.1016/j.jalgebra.2006.01.055. MR.2271575

[16] Ben Ford, Irreducible representations of the alternating group in odd characteristic, Proc. Amer. Math. Soc. 125 (1997), no. 2, 375-380, DOI 10.1090/S0002-9939-97-03621-6. MR:1353385

[17] Ben Ford and Alexander S. Kleshchev, A proof of the Mullineux conjecture, Math. Z. 226 (1997), no. 2, 267-308, DOI 10.1007/PL00004340. MR1477629

[18] The GAP Group, GAP groups, algorithms, and programming, http://www.gap-system.org.

[19] Roderick Gow and Alexander Kleshchev, Connections between the representations of the symmetric group and the symplectic group in characteristic 2, J. Algebra 221 (1999), no. 1, 60-89, DOI 10.1006/jabr.1999.7943. MR.1722904

[20] John Graham and Gordon James, On a conjecture of Gow and Kleshchev concerning tensor products, J. Algebra 227 (2000), no. 2, 767-782, DOI 10.1006/jabr.1999.8247. MR 1759843

[21] G. D. James, On the decomposition matrices of the symmetric groups. II, J. Algebra 43 (1976), no. 1, 45-54, DOI 10.1016/0021-8693(76)90143-5. MR430050

[22] G. D. James, The representation theory of the symmetric groups, Lecture Notes in Mathematics, vol. 682, Springer, New York/Heidelberg/Berlin, 1978.

[23] Gordon James, The representation theory of the symmetric groups, The Arcata Conference on Representations of Finite Groups (Arcata, Calif., 1986), Proc. Sympos. Pure Math., vol. 47, Amer. Math. Soc., Providence, RI, 1987, pp. 111-126. MR933355

[24] Gordon James and Adalbert Kerber, The representation theory of the symmetric group, Encyclopedia of Mathematics and its Applications, vol. 16, Addison-Wesley Publishing Co., Reading, Mass., 1981. With a foreword by P. M. Cohn; With an introduction by Gilbert de B. Robinson. MR644144

[25] Christoph Jansen, Klaus Lux, Richard Parker, and Robert Wilson, An atlas of Brauer characters, London Mathematical Society Monographs. New Series, vol. 11, The Clarendon Press, Oxford University Press, New York, 1995. Appendix 2 by T. Breuer and S. Norton; Oxford Science Publications. MR.1367961

[26] Jens C. Jantzen and Gary M. Seitz, On the representation theory of the symmetric groups, Proc. London Math. Soc. (3) 65 (1992), no. 3, 475-504, DOI 10.1112/plms/s3-65.3.475. MR 1182100

[27] Alexander S. Kleshchev, On restrictions of irreducible modular representations of semisimple algebraic groups and symmetric groups to some natural subgroups. I, Proc. London Math. Soc. (3) 69 (1994), no. 3, 515-540, DOI 10.1112/plms/s3-69.3.515. MR.1289862

[28] A. S. Kleshchev, Branching rules for modular representations of symmetric groups. III. Some corollaries and a problem of Mullineux, J. London Math. Soc. (2) 54 (1996), no. 1, 25-38, DOI 10.1112/jlms/54.1.25. MR1395065

[29] Alexander Kleshchev, On decomposition numbers and branching coefficients for symmetric and special linear groups, Proc. London Math. Soc. (3) 75 (1997), no. 3, 497-558, DOI 10.1112/S0024611597000427. MR 1466660

[30] Alexander Kleshchev, Linear and projective representations of symmetric groups, Cambridge Tracts in Mathematics, vol. 163, Cambridge University Press, Cambridge, 2005, DOI 10.1017/CBO9780511542800. MR2165457

[31] Alexander Kleshchev, Lucia Morotti, and Pham Huu Tiep, Irreducible restrictions of representations of symmetric groups in small characteristics: reduction theorems, Math. Z. 293 (2019), no. 1-2, 677-723, DOI 10.1007/s00209-018-2203-1. MR4002296

[32] Alexander Kleshchev and Vladimir Shchigolev, Modular branching rules for projective representations of symmetric groups and lowering operators for the supergroup $Q(n)$, Mem. Amer. Math. Soc. 220 (2012), no. 1034, xviii+123, DOI 10.1090/S0065-9266-2012-00657-5. MR 3014600 
[33] Alexander S. Kleshchev and Jagat Sheth, Representations of the alternating group which are irreducible over subgroups, Proc. London Math. Soc. (3) 84 (2002), no. 1, 194-212, DOI 10.1112/S002461150101320X. MR1863400

[34] Alexander S. Kleshchev and Pham Huu Tiep, On restrictions of modular spin representations of symmetric and alternating groups, Trans. Amer. Math. Soc. 356 (2004), no. 5, 1971-1999, DOI 10.1090/S0002-9947-03-03364-6. MR2031049

[35] Stuart Martin, Schur algebras and representation theory, Cambridge Tracts in Mathematics, vol. 112, Cambridge University Press, Cambridge, 1993, DOI 10.1017/CBO9780511470899. MR:1268640

[36] Lucia Morotti, Irreducible tensor products for symmetric groups in characteristic 2, Proc. Lond. Math. Soc. (3) 116 (2018), no. 6, 1553-1598, DOI 10.1112/plms.12127. MR3816389

[37] Lucia Morotti, Irreducible tensor products for alternating groups in characteristic 5, Algebr. Represent. Theory 24 (2021), no. 1, 203-229, DOI 10.1007/s10468-019-09941-0. MR4207396

[38] Lucia Morotti, Irreducible tensor products for alternating groups in characteristics 2 and 3, J. Pure Appl. Algebra 224 (2020), no. 12, 106426, 32, DOI 10.1016/j.jpaa.2020.106426. MR 4099917

[39] Lucia Morotti, Composition factors of 2-parts spin representations of symmetric groups, Algebr. Comb. 3 (2020), no. 6, 1283-1291, DOI 10.5802/alco. MR4184046

[40] A. O. Morris, The spin representation of the symmetric group, Proc. London Math. Soc. (3) 12 (1962), 55-76, DOI 10.1112/plms/s3-12.1.55. MR136668

[41] G. Mullineux, Bijections of p-regular partitions and p-modular irreducibles of the symmetric groups, J. London Math. Soc. (2) 20 (1979), no. 1, 60-66, DOI 10.1112/jlms/s2-20.1.60. MR.545202

[42] M. H. Peel, Hook representations of the symmetric groups, Glasgow Math. J. 12 (1971), 136-149, DOI 10.1017/S0017089500001245. MR308249

[43] Aaron M. Phillips, Restricting modular spin representations of symmetric and alternating groups to Young-type subgroups, Proc. London Math. Soc. (3) 89 (2004), no. 3, 623-654, DOI 10.1112/S0024611504014893. MR2107009

[44] John R. Stembridge, Shifted tableaux and the projective representations of symmetric groups, Adv. Math. 74 (1989), no. 1, 87-134, DOI 10.1016/0001-8708(89)90005-4. MR.991411

[45] David B. Wales, Some projective representations of $S_{n}$, J. Algebra 61 (1979), no. 1, 37-57, DOI 10.1016/0021-8693(79)90304-1. MR.554850

[46] Ilan Zisser, Irreducible products of characters in $A_{n}$, Israel J. Math. 84 (1993), no. 1-2, 147-151, DOI 10.1007/BF02761696. MR.1244664

Institut Für Algebra, Zahlentheorie und Diskrete Mathematik, Leibniz Universität Hannover, 30167 Hannover, Germany

Email address: morotti@math.uni-hannover.de 